\title{
Ueber die Einrichtung des Neudrucks, insbesondere über die Druckfehler und Varianten bei Leiçarraga.
}

Die von uns neu veröffentlichten Texte werden durch drei verschiedene Drucke aus dem Jahre 1571 gebildet, über welche J. Vinson in seiner ausgezeichneten baskischen Bibliographie von 1891 und 1898 (hier kurz mit $B B$ angeführt) unter 3 und 4 in erschöpfender Weise berichtet. $E$ s sind: das Neue Testament, Testamentu berria, mit Zubehör (abgekürzt $T B$; Bogen *, **, ${ }^{* * *} \mathrm{I}$ bis $\mathbf{I V}-a$ bis $z, A$ bis $Z, A a$ bis $M m \mathrm{IV}$; diese, das letzte Blatt ausgenommen, und nur diese mit Blattzahlen, nach denen ich immer zitiere $-\tilde{a}$ bis $\tilde{o}$ vII $-A$ bis $G$ ), der Kalender (Bogen a) und das Abc (Bogen $A$ bis $L$ ). Der Inhalt von $B$ VIII bis $G$ VII (ohne Blattzahlen) im TB deckt sich mit dem von $B$ VII bis $H$ VI, $K I I$ bis $L$ VI im Abc; wir geben den Text des ersteren wieder, und ich verzeichne die Abweichungen des letzteren im Anhang zu dieser Einleitung.

Von alledem ist bisher nur sehr Weniges im Neudruck erschienen. Bei den Bruchstücken die FL. Lícluse 1826 (1874) und 1831 (BB 201 und 3 h) bekannt gemacht hat, dem Gleichniss vom verlorenen Sohn und der Bergpredigt, kam es ihm selbst anf strenge Gensuigkeit der Wiedergabe nicht an. Eine solche hat sich, so viel ich sehe, zuenst C. A. F. MAms. in seinen „Denkmälern" von 1857 (BB 296) S. 1-12 (Luk. XV, 11-32. Matth. II-V. Apostelg. I-III) vorgesetzt und auch fast ganz erreicht. Seine Leistung verdient um so grössere Anerkennung als der hier beliebte Druck der denkbar unerfreulichste und ermüdendste ist und den Eindruck macht geradezu eine Brutstātte für alle möglichen Druckfehler zu. bilden. Wenn ein psar kleine Abweichungen, wie das Fehlen eines Akzentes (M. II, 7. Ap. III, 17) oder eines Bindestrichs (M. IV, 17), die Auflösung einer Tilde-abkürzung (M. III, 15. Ap. II, 14) vorkommen, so besagt das eben nur dass auch der angestrengtesten Sorgfalt gewisse Grenzen gesetzt sind. Fine Inkonsequenz liegt allerdings darin dass - Mahn zwei Druckfehler, doppeltes işa $(M . V, 21)$ und $c$ für $q(M . V, 16)$ verbessert, einen dritten, wiederum $c$ für $\varsigma^{\prime}(\mathbf{M}$. II, 8) belässt. 
J. VINson ist der Erste welcher einen abgeschlossenen Theil des TB neu herausgegeben hat, nämlich 1874 das Markusevangelium und zugleich damit die französische und baskische Widmung an die Königin Johanna (BB 3b). Aber er hat diese Aufgabe nicht so gelöst wie man von ihm erwarten durfte, der damsls wie unter den Basken selbst, so auch schon im Baskischen ganz zu Hause war und der von jeher auf die kleinsten und allerkleinsten Dinge seine Aufmerksamkeit zu richten liebte. Seiner Ausgabe, „entièrement conforme a la première de $1571^{\text {" }}$ (80 der Titel), lag eine "copie manuscrite à peu près complète" (Bo die Vorrede) zu Grunde, welche ungefähr funfzig Jahre alt war und zwar wie es scheint recht nachlässig angefertigt (vgl. Rev. de ling. VII, 282 f.). Vinson hat die Randvermerke (die Parallelstellen) weggelassen und die schräge Schrift der steilen, die $\int$ den 8 gleich gemacht. Hingegen hat er die Tildes beibehalten wollen, was nur bei gleichen Zeilenenden einen Sinn gehsbt hätte; thatsächlich sind sie aber, besonders gegen den Schluss zu, sehr oft (wohl ein $\mathrm{Halbhundert} \mathrm{Mal}$ ) aufgelöst. Noch weniger verstehe ich welcher $Z$ weck damit verfolgt werden sollte dass kein Wort am Ende der Zeile abgebrochen wurde; dem haben wir zwei gesperrte Zeilen (I, 30 . XIV, 54) zu verdanken. Die Abweichungen von der Interpunkzion des Urdrucks belaufen sich auf gegen hundert, und was weit wichtiger ist, die Akzente fehlen in halb so vielen Fällen und sind wiederum nicht selten überflüssig oder falsch gesetzt. Von Geringerem, wie den Tremas, den grossen und kleinen Buchstaben, der Worttrennung nicht zu reden. Was die Druckfehler im engsten Sinne anlangt, so hat sich Vinson erst sehr spät entschlossen ein Verzeichniss derselben zu liefern, nämlich in der BB S. 39; es besteht aus 45 Druckfehlern innerhalb der einzelnen Wörter und den Auslassungen von 21 Wörtern. Aber das ist jedenfalls nur die Hälfte, ich habe nämlich ausserdem 60 Fehler der ersten und 10 der zweiten Art gefunden; ich beschränke mich auf die Angabe der letzteren: bat fehlt III, 20, campora III, '23, berriz IV, 1, Eta(e-) IV, 17. V, 6. XII, 19.21. 30, du XII, 37, Harc XVI, 10. Wenn unter den andern sehr viele sind von denen man schwer begreift wie sie einem Basken - ein solcher las die Korrektur nach Vinson - zu entgehen vermochten, so finden sich wiederum solche die nur von einem Basken herrïhren können, z. B. iduri für irudi, permetatea für fermetatea. Der Urdruck hat XIV, 27: fcandalizaturen sarete nitan; Vinson bietet dafür: .... niçaz; das ist heutiger Sprachgebrauch, ganz 80 wie man im Französischen heute sagt: vous serez 
scandalizés de moi statt: .... en moi, welches die Bibel des 16.Jhrhs, hat. Manche Druckfehler des Urdrucks sind berichtigt; andere wiederum nicht. Nach dem Ermittelten kommen bei Vinson auf den Bogen des Urdrucks etwa 23 Buchstabendruckfehler und 7 fehlende Wörter. Von der Widmung sehe ich hierbei ab, deren baskischer Text trotz einiger starken Versehen (occasine statt oceafĩone, coferitu statt côferitu hat Vinson auch nachträglich nicht bemerkt) mit mehr Sorgfalt behandelt worden ist als der französische.

Dieser Ausgabe des Markusevangeliums ist die von W. J. VAN EYs 1877 veranstaltete des Matthäusevangeliums (BB 3c) dem Plan und der Ausführung nach bedeutend uberlegen. Die Randvermerke sind hier mitgedruckt; die Schrägschrift (ausser in den Randvermerken), die $f$, die Buchstabenverbindungen (wie $c t, \Omega$ ) des Urdrucks sind gewahrt, nicht die Tilde-abkürzungen. Die Seiten decken sich einigermassen mit denen des Urdrucks, sber durchaus nicht so dass die von van Eys beliebte Blattzählung gerechtfertigt erschiene. In dem kurzen Vorwort heisst es dass die offenbaren Druckfehler berichtigt worden seien; das ist nur zum Theil der Fall, go druckt auch van Eys: iffafforraco IV, 15, paffíonea XVII Ü., lekorrerat XXV,15, ohiu XXVII, 23. Ueber die Grundsätze die in der Anwendung der Beizeichen obwalten, spricht auch er sich nicht aus; jedenfalls sind da manche Versehen festzustellen. Das am Schluss gegebene Druckfehlerverzeichniss (in dem übrigens wiederum ein Halbdutzend Druckfehler stecken) umfasst deren 37 - ich sehe hier wie überhaupt von den falschen Zahlen der Randvermerke ab -, reicht aber nur bis XVI, 17; ich weiss nicht warum nicht weiter, warum nicht wenigstens bis ins XXIV. Kapitel, da erst gegen Ende dieses die beiden letzten Bogen anheben, deren Korrektur van Eys nicht mehr in eigener Person hat besorgen können. Vinson BB S. 40 fügt 10 hinzu; aber er giebt zweinual die Schreibung ron van Eys selbst: erofleac (statt erofleac) und promettatu, und im letzteren Falle handelt es sich um die Beseitigung von einem Druckfehler des Urdrucks (prometatu), ebenso wie bei chikiratu statt chilkiratu, in einem andern Fali endlich liegt nur eine Zusammenrückung zweier Wörter vor. Diese 44 Druckfehler, von denen einer in dem Ausfall eines Wortes besteht, haben mir eine Nachlese von etwa 30 Buchstabendruckfehlern und dem Ausfall von 6 Wörtern (XXV, 24) gelassen. Demnach kommen bei van Eys auf den Bogen des Urdrucks etwa 10 Buchstabendruckfehler und 1 ausgefallenes Wort. Auf einer von van Eys eingesandten Abschrift des 


\section{XII}

Johannesevangeliums beruht der Abdruck von dessen ensten zehn Kapileln in der "Euskara“ S. 66 ff. 75 ff. 85 f. 94 ff. 103 ff. 117 ff. 122 f. 140 f. (N. 9-15. 17; 15. April 1891 bis 1. Juli 1896). Auch hier kommen auf den Bogen des Urdrucks etwa 10 Buchstabendruckfehler; in den "Corrigenda" zu den ersten sechs Kapiteln ebend. S. 105 f. (N. 13) hat Dodgson nämlich ein Dutzend solcher übersehen. Hier ist übrigens hitiric IV, 30 in hiriric falsch korrigiert (statt hiritic); und Baldi III, 12, discipuluequĩ III, 22 waren überhaupt nicht $\mathrm{zu}$ korrigieren (die steile in schräge Schrift).

In der "Euskara" hat auch E. S. Dodgson selbst verschiedene Stǘcke abgedruckt. Zunächst S. 55 f. (N. 7; 1 . Dez. 1889 ) den Brief von Judas, und S. 60 f. (N. 8; 1. Juni 1890) den an Philemon, beide mit der Zeilentrennung des Urdrucks; in ersterem finden sich 6 Fehler, darunter der Ausfall eines Wortes (S. 105 hat Dodgson das richtige Core-erén in Core-eren und tenebrataco falsch in tenebrateco korrigiert), in letzterem keiner. Endlich hat Dodgson in der "Euskars ${ }^{2}$ S. 87 (N. 11; Ende März 1892) auch S. * VI' des 'TB abgedruckt, nicht sehr genau (S. 105 ist nicht Alles berichtigt, selbst ecen behingoaz nicht in ecin b.), dann, 1895 , in der 2 . Etude sur la langue Euskara S. 8 viel besser, und in der Euskal-erria XXI (1889, II), $179 \mathrm{ff}$. jene drei Seiten Erklärungen fremder Namen die unmittelbar auf die Apokalypse folgen, mit über zwei Dutzend Fehlern.

Dodgson hat $\mathrm{zu}$ verschiedenen kleineren Theilen des TB Konkordanzen, oder wie er sie Bonst nennen mag, an verschiedenen Orten veröffentlicht, Bo auch zu den Petrusbriefen in der Rev. de ling. XXII, 167-181. 262-277 und dazu wieder eine lange Liste von "Additions et corrections " ebend. XXIV, 43-48, aus denen die Redakzion weggelassen hat was ihr zu wenig bedeutend erschien. Hier werden nun verschiedene Abschreibfehler Dodgsons ausdrücklich als Druckfehler des Urdrucks bezeichnet. Zu gaiç I, IV, 3 bemerkt er: „ls seconde cédille de gaiçu est une faute d'impression originale, qu'il n'est pas nécessaire de répéter dans une concordance". (S. 43); der Urdruck hat çaicu. Zu harģaz II, II, 14: „orig. harcaz" (S. 44); der Urdruck hat harçaz. Zu emendamendutara II, III, 9, wie er dem Urdruck gemäss XXIII, 178 zitiert: lisez emendamentura " (S. 46); man muss denken dass so im Urdruck steht, denn als sprachliche Verbesserung wäre es ganz unberechtigt (vgl. einerseits das à repentance, nicht $\dot{a}$ la $r$. des französischen Textes an dieser Stelle, anderseits emendamen. dutara $=$ en oder $\dot{a}$ repentance Matth. III, 11. Luk. V, 32. 
Röm. II, 4; das Wort lautet ja auch emendamendu, nicht emendamentu). Harcaz I, I, 21 hatte Dodgson XXIII, 266 gelesen; er bemerkt dazu: "pour Harcaz sic orig., lisez hargaz" (S. 47); der Urdruck hat hargaz. Lehenago danic II, II, 5 hatte Dodgson XXIII, 269 gelesen; er berichtigt: lisez lehenagoda. nic. Hautin l'a divise ${ }^{4}$ (8. 47); der Urdruck hat lehenagodanic i. e. W. $\mathrm{Zu}$ ohoraitzague I, II, 17 heisst es: ${ }_{n}$ lisez ohoritzaçue (S. 48); der Urdruck hat ohoraitzague. Çaretençát II, II, 2 (XXIII, 172) soll durch garetencát ersetzt werden (S. 44); der Urdruck hat sogar caretencat. In The Basque Verb found and defined, Second part (Extr. des Actes de la Soc. Philol. XXV, 1896) S. 43 hat D. unter den "Corrigenda" zum ersten Theil (ebd. XXII) zu Joh. I, v, 10 (teftificatu vkan duen): „Hautin a mis un point après vkan ; nein, nicht nach diesem vkan, sondern nach dem der vorhergehenden Zeile, das war aber schon I, 31 bemerkt worden.

Die Fehltritte unserer Vorgänger haben uns zar Warnung und Weisung gedient. Ein älteres Schriftwerk kann auf dreierlei Weise neu gedruckt werden, entweder so wie es ist, oder so wie es sein Urheber wollte, oder so wie es der heutige Leser braucht. Dass das Letzte für uns ganz ausgeschlossen war, bedarf keiner Darlegung. Ich bemerke beiläufig dass eine Modernisierung des Leiçarragaschen TB aus mehr und stärkeren Aenderungen bestehen müsste als die sind welche Axulars freilich weit jüngeres Erbauungsbuch (1642) durch Inchauspe (1864) erfahren hat; denn die Sprache Leicarragas liegt den heutigen Labourdern und Niedernavarrern mindestens ebenso fern als die Sprache Luthers einem heutigen Deutschen. Anderseits ist das baskische N.T. welches $18<8$ unter den Fittichen der englisch-protestantischen Propaganda erschienen ist (ich kürze ab: $T B^{\text {ro }}$, etwas mehr als eine sprachliche Modernisierung, als eine ${ }_{n}$ Retouche ${ }^{4}$ welche Vinson darin erblickt, der Uebersetzung von 1571; sie weicht stark genug von ihr ab um nicht unmittelbar an sie angegliedert zu werden, wie das in der BB unter 38 geschieht (wo aber die Wiederabdrucke des Lukasevangeliums unter 381 davon getrennt sind). Das TB besitzt nur die Bedeutung eines Sprachdenkmals. Es erhob sich nun die Frage ob wir die Druckfehler die ihm anhaften, belassen oder ob wir sie entfernen, das heisst, das Werk so herstellen sollten wie es der Herausgeber haben wollte. Von dieser Frage löste sich als Vorfrage ab ob wir das konnten und zwar im vollen Umfange konnten. Die Druckfehler eines Buches werden entweder an dessen Schluss vom Herausgeber selbst verzeichnet, oder sie ergeben sich sus der Kenntniss die 


\section{XIV}

wir von seiner Absicht und zugleich von der gelieferten Vorlage haben. Der letztere Fall ist ziemlich selten, in ihm befindet sich unsere Veröffentlichung selbst; der andere ist weit häufiger. Am häufigsten jedoch ist uns eine sichere und vollständige Feststellung der Druckfehler gar nicht möglich. Zu einer solchen reicht die äussere Betrachtung nicht aus; als Malherbes Et Rosette a vécu vom Setzer, mit oder ohne Bedacht, in Et rose, elle a vécu umgeändert wurde, war dieses ein Druckfehler, hörte aber auf es zu sein als der Dichter, zu seinem Ruhme, sich damit einverstanden erklärte. Nun gibt es allerdings unbedingt falsche Druckweisen, und wo wir sicher sind dass sie nicht irgend welcher Schrulle oder einem lehrhaften Zwecke ihren Ursprung verdanken, haben wir sie als Druckfehler anzusprechen. Aber ihre Zahl ist weit beschränkter als es auf den ersten Blick scheint; die beiden Richtschnuren die uns zur Bestimmung des Falschen dienen, lassen uns bald im Stich. Im Allgemeinen glauben wir zu leicht den Sinn des Zusammenhangs zu verstehen und schicken uns zu rasch an das $\mathrm{zu}$ beseitigen was ihn zu stören scheint. Was das Andere anlangt, die Sprachrichtigkeit, so durchdringt sie nicht einmal unsere so sorgfältig gepflegten Schriftsprachen in allen Faseru, bis in alle Spitzen. Insbesondere die deutsche weist zahlreiche, obschon in ihrem Werthverhältnisse sehr ungleiche Varianten auf, und auch das einzelne Schriftwerk vermag sich kaum davon frei $\mathrm{zu}$ halten - ich will sie dann innere Varianten nennen. Finden wir nun z. B. Hülfe sehr oft und Hilfe nur ausnahmsweise gedruckt, so dürfen wir das Letztere mit $\mathrm{Zu}$ versicht als Druckfehler ansehen; ferner einen solchen ebenfalls roraussetzen wo wir je ein Mal Hülfe und Hilfe lesen, nur dass wir nicht wissen wo cr liegt; stossen wir jedoch auf einen unterschiedslosen Nebeneinandergebrauch, $\mathbf{z}$. B. von unseren und unsern, so werden wir das auf die Gleichgültigkeit des Schriftstellers zurückführen, wie sehr er in andern Dingen sich selbst scharf auf die Finger sehen mag. In je frühere Zeiten, in je entlegenere Sprachgebiete wir emporsteigen, ein um so geringerer Eifer für die sprachliche Einheitlichkeit wird uns meistens in den Druckwerken entgegentreten, um so unsicherer werden wir uns in der Beurtheilung der inneren Varianten fühlen. Leicarraga hat die Sprache in der er geschrieben, selbst festgestellt. Er sagt zwar in der Widmung nur dass das Baskische bis dahin, wenigstens in Uebersetzungen, ungebräuchlich gewesen sei; aber auch an originaler Litteratur war gewiss Nichts vorhanden an das er hätte anknüpfen können. Es gab einen einzigen älteren Druck in baskischer Sprache. Dechepares Ge- 
dichte von 1545 und 2 war von geringem Umfang, in nachlässigster Form, zudem in einer andern Mundart als der von Leicarraga zu Grunde gelegten, sodass sie für diesen, wenn er sie überhaupt kannte, ohne Bedeutung blieben. Wie sehr nun auch Leiçarraga, was gerade aus dem Vergleich mit Dechepare hervorgeht, sich um die Sprache bemühte, so macht es doch der bezeichnete Umstand begreiflich dass seine Schriften eine hohe Zahl von Varianten enthalten, und ihre Beurtheilung wird uns dadurch erschwert dass es uns an gleichzeitigen Werken gleicher mundartlicher Färbung fehlt. Wir sind in manchen Fällen (bataye, dracun u. s. w.; s. unten) nicht einmal darüber einig ob es sich um eine mundartliche Nebenform oder um einen mechanischen Druckfehler handelt. Besondere Verlegenheit würde uns die Setzung der Akzente bereiten. Kurz, welche Grundsätze für die Bestimmung dessen was als Druckfehler zu betrachten sei, wir uns auch, sicherlich nicht ohne Widerspruch, hätten aneignen mögen, wir konnten die Texte nicht ohne Druckfehler, und daher mussten wir sie mit allen Druckfehlern wiedergeben. Das schloss natürlich die Verpflichtung zu einem kritischen Berichte über diese in sich; sie wäre aber noch unabweislicher gewesen wenn wir irgend einen Versuch unternommen hätten die Druckfehler auszumärzen. Man hat im Allgemeinen die Bedeutung dieser Angelegenheit unterschätzt. Vinson sagt BB S. 7 vom TB: „L'exécution typographique est très soignée et la correction surprenante" (fast derselben Worte hatte er sich schon vor dem Markusev. S. III f. bedient) und gibt vier Druckfehler mit einem ,etc. an, wozu er noch vier Versehen in der Seitenzählung fügt. S. 522 trügt er zwei Fehler jener und einen dieser Art nach. Van Eys sagt Les Verbes auxiliaires dans le Noureau Testament de Liçarrague, 1890 (BB 673; ich kürze es mit VAL ab) S. 19: ${ }_{n}$ Les erreurs de ce genre [les erreurs typographiques] sont extrèmement rares dans le précieux volume de ligarrague, qui est imprimé arec des soins inouis; cependant il $y$ en a quelques unes." In der Anmerkung dazu verzeichnet er neunzehn Druckfehler, darunter abcr das richtige ligunçat und ein paar mundartliche Formen, in den "Additions ${ }^{4}$ vor S. 1 kommen noch drei hinzu. Wie man ersehen wird, zählen die Druckfehler bei Leiçarraga nicht nach Dutzenden, sondern nach Hunderten, und das ist für die Beurtheilung mehrdeutiger Druckweisen von grosser Wichtigkeit. Bei alledem müssen wir das TB als eine gute Druckleistung anerkennen, die hoch über die andern baskischen Drucke dieses und des folgenden Jahrhunderts emporragt. Es wäre auch für unser Interesse 


\section{XVI}

nicht ganz zwecklos - doch habe ich mich dieser Aufgabe nicht unterziehen können - festzustellen wie sich in Bezug auf die Zahl der Druckfehler zu diesem baskischen das französische Neue Testament verhält das sechs Jahre opäter ebenfalls zu La Rochelle und von demselben Haultin (80 schreibt er sich hier) gedruckt wurde. Der Titel lautet: LE | NOVVEAV | TESTAMENT, | C'EST 'A DIRE, | LA NOVVELLE AL- | liance de no/re Seigneur | IESVS CHRIST. | A LA ROCHELle. | Par Pierre Hadttio. | 1577. Vinson, in dessen Exemplar des Titelblatt fehlt, sagt davon BB S. 37: ,les csractères sont en effet les mêmes que ceux du livre basque de 1571, mais l'impression est bien moins soignée." Das Erstere ist jedenfalls unrichtig; die Typen des Haupttextes sind hier kleiner als dort. Von den Irrthümern in der Blattzählung die Vinson angibt, finden sich zwei in meinem Exemplar nicht: „197 manque, 220 pour $203^{\prime \prime}$. Ich werde mich bei verschiedenen Gelegenheiten auf dieses franz. N.T. von 1577 beziehen (manchmal auch auf das von 1563, Genf, Iean Bonne-foy; abgek.: franz. ${ }^{77}$, franz. ${ }^{65}$.

Das Bequemste, Schnellste und Sicherste würde die Vervielfältigung des Urdrucks auf mechanischem Wege gewesen sein. Allein sie war für uns durch dic unumgängliche $\mathrm{Er}$ haltung und Schonung der zur Verfügung gestellten Exemplare ausgeschlossen. Wir mussten Alles neu setzen lassen. Die kleinen Unvollkommenheiten welche bei diesem Verfahren nicht zu vermeiden gewesen sind, werden wohl der wissenschaftlichen Benutzung keinen Eintrag thun und durch die grössere Schärfe und Lesbarkeit des Druckes aufgewogen werden. Der Mühe aber die wir selbst aufgewendet haben, verdanken wir eine Vertrautheit mit der Sprache Leiçarragas von der wir hoffen dass sie auch Andern zugate kommen wird - nicht bloss und nicht am Meisten durch diese zum grossen Theil ja nur äusserlichen Dingen gewidmete Einleitung.

Sobald wir uns vorgesetzt hatten alle Druckfehler wiederzugeben und zugleich genau die Umstände unter denen sie auftreten, mussten wir den Text gerade so vertheilen wie den Urtext, das heisst diesem Seite für Seite, Zeile für Zeile folgen. Nebenbei will ich bemerken dass im Allgemeinen jede Seite des Haupttextes, von denen mit Kapitelüberschriften abgesehen, 31 Zeilen enthält, manche aber vor einer Kapitelüberschrift, damit diese nicht gebrochen werde (obwohl dieses oft genug geschieht, z. B. $\left.113^{\circ}, 131^{1}, 163^{r}, 216^{\prime}\right), 30$ und weniger Zeilen (70 sogar bloss 24), ein paar auch vor der Mitte des 
Kapitels 30 (80 28', 90'), anderseits ganz wenige 32, indem der Versechluss neben die Signatur gesetzt ist, so $324^{\mathrm{r}}, 356^{\mathrm{r}}$, $396^{\prime}$, und im letzten Falle wurde die Verlängerung auch auf die gegenüberliegende Seite $395^{\prime}$ übertragen. Ferner haben ${ }^{* *} \mathrm{II}^{r}, \mathrm{VI}^{r}, \mathrm{VII}^{r}$, $\mathrm{III}^{1}, \mathrm{IV}^{r}, \mathrm{G} \mathrm{III}^{1} 40$ Zeilen statt 39 . Der im Allgemeinen nicht seltene Fehler der Silbenwiederholung im Zeilenbruch findet sich hier nur ausnahmsweise: heta-| taric $=$ heta $\mid$ ric $146^{\mathrm{r}} 1$.

Die Seitenüberschriften stimmen nicht immer zum Inhalt der Seite, so:
CAP. XXI.
CAP. IX.
- CAP. XX. 249\%.
CAP. X.
CAP. X. 304.
CAP. XX.
CAP. IX. $441^{\circ}$.
CAP. XXII.
CAP. XXI. $456^{r}$.
S. PIERRIS. I.
CAP. XXI. $457 \%$.
Man bemerke noch: TTMOTHEO.
S. PIERRIs. II. 417 '. 418 '.
Fehler in der Seitenzählung, wie:

$\begin{array}{ll}105 & 205 \\ 106 & 206 \\ 108 & 208 \\ 438 & 448\end{array}$

sind allerdings nicht relativer Natur, ihre Wiedergabe würde aber in einem Drucke der die Seiten anders ordnet als der Urdruck, keinen Sinn haben. Aus der getreuen Nachbildung der Seiten und Zeilen des Urdrucks erwuchs uns übrigens der Vortheil dass wir unsern Druck leichter und sicherer nachvergleichen konnten.

Die ganz gleiche Höhe und Breite der Seiten wie im Urdruck herzustellen, war uns nicht verstattet. Die Seite des Haupttextes z. B. ist (ohne Kolumnentitel, Signatur und Randschrift) bei uns $3,5 \mathrm{~mm}$ höher und $4 \mathrm{~mm}$ breiter. Da nun aber die beiderseitigen Lettern durchschnittlich die gleiche Breite haben, wovon man sich durch das Ausmessen ganzer Wörter am besten überzeugen kann, so ist unser Druck viel splendider ausgefallen, das heisst, weist stärkere Ausschliessungen auf. In $\tilde{a}-\tilde{o}$ ist unsere Halbseite nicht einmal $1 \mathrm{~mm}$ breiter, zugleich sind aber unsere Buchstaben etwas kleiner, und in Folge dessen liegen die Wörter auffällig weit auseinander. So weicht denn im Allgemeinen unser Druck wesentlich von dem, damaliger Sitte gemäss, mehr oder weniger eng gehaltenen Urdruck ab. Die Wörter sind hier grossentheils nur durch Haarspazien voneinander getrennt, und wenn sogar diese nicht 


\section{XVIII}

selten fehlen, so beruht das hauptsächlich auf einer Bequemlichkeit oder Gleichgültigkeit die sich auch in andrer Beziehung äussert, so in dem beliebigen Weglassen des Theilungszeichens. Solche zusammengebackenen Wörter dürfen demnach nicht so beurthcilt werden wie die welche sich, selten genug, in den heutigen Drucken finden. Uebrigens gibt es Falle in denen die Worttrennung gar keines Spaziums bedarf, sondern das zweite Wort in seinem Beginn durch eine Versalie oder durch $v$ (das nie in der Mitte erscheint) oder in seiner Gänze durch die Wahl einer andern Schriftart kenntlich wird, z. B. ceneanBazco $163^{\prime} 23$, recebituvkan $43^{\prime} 27$, denacbere $258^{\prime} 16$. Die Nachahmung eines derartigen Zusammenrückens in unserem Druck muss ein falsches Bild von den Verhältnissen des Urdrucks erzeugen, und wenn wir sie doch hie and da vorgenommen haben, so ist der Grund der dass wir nicht sofort uns getrauten von diesen Fällen diejenigen zu scheiden in denen die Absicht bestand eine Worteinheit darzustellen (diese werden unten, gelegentlich des Bindestrichs, besprochen werden) und dass auch späterhin Manches besonders auffällig war. Zuweilen wiederum sind Wörter auseinander gerissen worden, und auch da sind wir uns leider nicht treu geblieben, wir haben, obwohl ja hier an eine sprachliche Bedeutung kaum zu denken ist, das bald wiedergegeben (z. B. cira denean 1'11), bald nicht (z. B. hun á 14'3). Ebenso sind wir da verfahren wo ein Buchstabe von einem Worte zum andern hinūbergeschoben ist; wir haben z. B. wiedergegeben ce rokanen ê I'a30, aber nicht complituI a i II'a 11 . Was die Interpunkzionszeichen betrifft, so pflegten nicht bloss wie heute der Punkt, sondern auch der Doppelpunkt, das Fragezeichen und der Beistrich, und zwar auch vor einem leeren Zeilentheil, dicht an das vorhergehende Wort herangerückt zu werden. Nicht selten erscheinen sie zwischen zwei Wörter geradezu eingekeilt, anderseits werden sie dann und wann weit abgerückt, z. B. $C A P . I \cdot 98^{\circ}$. Auch hier ist das Eine wiedergegeben, das Andre nicht. - Gegenüber den Verschiebungen nach oben oder unten haben wir uns ähnlich verhalten; wo sie in grösserem Umfang auftreten (z. B. in den Anfängen der ersten 9 Zeilen von ('r ), haben wir sie nicht berücksichtigt, einzelne erhobene oder gesenkte Buchstaben zum Theil, z. B. bekatuén E vi'24. Am Ende der Zeilen rutschen die Interpunkzionszeichen leicht hinauf oder hinab, fast bis zur nächsten Zeile, z. B. ethorri' е̃ IV' b1 (im L.), betheac' i vI 'a 8, heb. 10.so. i vn' a 31, 1. cor. $\mathrm{J}^{\prime} \mathrm{a} 3$ (Alles nicht wiedergegeben). - Die Verkehrtstellung von Buchstaben wie IOAN $193^{\top}$ oben, prox? ?tmoa 
a vII'b 25 ist meistens nicht wiedergegeben worden, nur icpn G vi'24; sie ist bcsonders häufig, weil am wenigsten auffälig, beim $z$ (Bo aitzinetic ${ }^{* *} \mathrm{II}^{1} 32$, colpatzeco ${ }^{* *} \mathrm{VIII}^{\mathrm{r}} 33$, goazte ${ }^{* * *} \mathrm{III}^{1} 24$, centuzten $76^{\prime} 19$, litzaqueleno $180^{\prime} 18$, OTHOITZA A I ' Ü., bazcatic A III'2, buruez $\mathrm{A} \mathrm{v}^{\circ} 19$, aitzitic A vi'29, verthutez $\mathrm{BI}^{\mathrm{r}} 36$, aditzen Crvi 7 , sodass sich auch in unserem Druck, nach der letzten Korrektur, ganz unberechtigt ein $z$ umgestellt hat (neurtzeco 457 15). Für I - wie die regelmässige Gestalt der ersten arabischen Ziffer ist - finden wir zuweilen $\mathrm{I}$, so $84^{1} 27 \mathrm{R}$. 149'14 R.

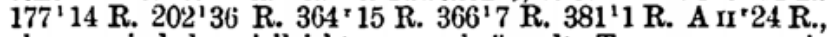
aber es sind das vielleicht nur verkrüppelte Typen ron $\mathbf{I}$, wie auch die aufwärts stehenden 1: $98^{1} 27$ R. $190^{1} 18 \mathrm{R} .2^{2} 3^{1} 3 \mathrm{R}$. 233'36 R. Frv'7 R. - Um es kurz zu sagen, man hat das was bei uns sich vom Regelmãssigen entfernt, was einen störenden Eindruck macht, unserer Vorlage zuzuschreiben (z. B. die eingerückten Verszahlen $1^{r}-11^{r}$, die wir, durch Uebersehen, bis $24^{\prime}$ fortgesetzt haben), aber nicht etwa anzunehmen dass jede äusserliche Besonderheit nachgebildet sei. Will man daraus einen Vorwurf für uns ableiten, so möge es nicht der sein Nothwendiges unterlassen, sondern Ueberflüssiges gethan zu haben. Wir mussten, damit Nichts ausgeschlossen werde was im Entferntesten von Bedeutung sein konnte, den Umkreis sehr weit ziehen; dass er selbst etwas zitterig und geschlängelt gerathen ist, bleibt gleichgültig und war nicht zu vermeiden, da wir vor dem buchstabenweisen Durcharbeiten dieser $z$ wölfhundert Seiten nicht Alles gewusst haben was wir hinterher wissen. - Hierzu kommt noch ein allerdings sehr nebensächlicher Umstand. Die verschiedenen Exemplare stimmen zwar grösstentheils, wie ja begreiflich, auch in den unwesentlichsten Dingen übcrein, aber doch nicht ausnahmslos. Das haben wir freilich nur gelegentlich und nur zwischen den von uns benutzten Exemplaren feststellen können. So hat das Stuttgarter und Leipziger $b i l^{\circ}, e d^{\circ} 412^{\prime} 3$ (nicht wiederg.), das L. weniggtens deus $428^{\prime} 1$ (wiederg.), das Berner bilo, edo, deus; so das L. 2.4 221 '3 R. statt Berriz 22.4 des St. und B. Sehr oft sind Punkte, zuweilen auch Akzente in dem einen Exemplar deutlich oder doch gerade noch sichtbar, in dem andern selbst mit der Loupe nicht zu erkennen (z. B. demborá St. $\sigma \mathbf{I}^{\prime} \mathbf{b} 29=$ dembora L.); wir haben über diese verblassenden Kleinigkeiten viel korrespondiert. Einmal habe ich für ein Trema angesehen was sich dann als ein schwarzes Element im Papier heraus stellte: diián 451'18. Im L. steht gaichtóa $137^{\prime}$ Ü., nicht im St.

Ueber den künstlerischen Theil des Neudrucks habe ich nicht viel zu sagen. In mechanischer Wiedergabe erscheint 
nur das Titelblatt und auf der folgenden Seite die Kopfleiste und die Iniziale. Die Inizialen an den Kapitelanfängen des TB, von denen $G$ und $H$ gegen den Schluss zu zum Theil durch einfache Versalien gewöhnlicher Grösse ersetzt werden $\left(435^{\prime}, 436^{\prime} .453^{\prime}, 455^{r}, 456^{r}, 457^{\prime}-346^{r}, 369^{\prime}\right)$, treten im Ganzen genommen, nicht jede einzelne, in dreifacher Grösse auf, indem sie der Höhe von rier, fünf und sieben Zeilen entspricht, und damit wechselt zugleich die Gestalt der Verzierung, z. B. $B$ 1) $410^{\circ}$, 2) $413^{\prime}$, 3) $321^{\circ}$. Wir haben Inizialen nur von 2weifacher Grösse gebracht, von denen die eine etwa der ersten des Urdrucks entspricht, die andere zwischen deren dritter und zweiter liegt. Und zwar kommen in der letzteren $H, N$ (z. B. $\left.317^{1}, 406^{\gamma}\right)$; in beiden $B, I\left(\right.$ z. B. $\left.321^{*}: 413^{r}, 1^{*}: 2^{\prime}\right)$, in der ersteren die übrigen Buchstaben $A, C, D, E, G, L, M I, O, P$, $R, S, V$ vor, und diese stimmen, $O$ und $R$ ausgenommen, in den Einzelheiten der Zeichnung ganz oder fast ganz mit den Inizialen zweiter Grösse des Urdrucks überein. In Bezug auf die Kopfleisten (insbesondere auch die im Kalender, welche der Urdruck in grosser Mannigfaltigkeit gibt) können wir uns eines ähnlichen Einklangs nicht rühmen, und noch weniger in Bezug auf die Schlussvignetten, da muss man uns sogar Stilwidersprüche hingehen lassen.

Im Urdruck sind vielfache Schriften verwendet worden, die wir, soweit die Mittel der Druckerei es gestatteten, wiedergegeben haben. Die verschiedenen Grössen, wo nicht völlige Deckung zu erzielen war, wenigstens in gleicher Abstufung. Wer die Schwierigkeiten einer solchen Bemessung kennt, wird einige Versehen bei uns entschuldigen. So sind die Lettern von $97 \times$ Zeile 3 kleiner als die der folgenden Zeile, während sie ebenso gross zu sein hätten; so hätten die von $367^{\circ}$ Zeile 4 und 5 kleiner als die von $374^{\prime}$ ' Zeile 4 und 5, nämlich kleinste Kapitälchen (wie auf dem Titelblatt Z. 3 v. u.) zu sein. Und in diesen war auch das CAP. I. $38^{\prime} 2^{\prime} .384^{\circ} .402^{\circ} .430^{\prime}$ zu setzen, während die jedesmal folgenden Kapitelüberschriften grössere Lettern aufweisen; und ein ähnliches Verhältniss findet sich auch sonst. Umgekehrt erscheint im Urdruck CAP. I. 353' mit grösseren Lettern als die folgenden Kapiteliiberschriften. Manchmal sind einzelne Buchstaben eines abweichenden Schriftgrades aus Versehen eingemischt, was wir meistens wiedergegeben haben, so Versalien in der Grösse der zugehörigen Minuskeln: ** $\mathrm{VII}^{*} 31.426^{1} 21$. ₹ $\mathrm{vI}^{*} \mathrm{~b} 36$, nicht wiederg.: Iefufec $16^{1} 36$; so eine kleinere Minuskel am Schluss eines Wortes wiederg.: $449^{\prime} 2$, eine grössere zu Beginn nicht wiederg.: Careten $284^{1} 9$. Das $P$ von $428^{\prime} 1$ ist nicht nur, was wir 
wiedergegeben haben, etwas erhöht, es ist zugleich kleiner als die umgebenden Majuskeln. - Der Unterschied in der Richtung der Schrift (steile, Antiqua und schräge, Kursiv) ist von uns streng gewahrt worden; nur waren wir gezwungen die kleine Schräggchrift die im Zubehör des TB mehrfach vorkommt ( $\mathrm{a}^{\mathrm{r}}, \mathrm{A} \mathrm{I}^{1} . \mathrm{IV}^{\prime}$ u. s. w.), durch Steilschrift (Nonpareille) zu ersetzen, weshalb wiederum die steilen Buchstaben in dela $\mathrm{Av}^{\mathrm{r}} 4$, Eligs $\mathrm{BII}^{\mathrm{r}} 38$ nicht hervorgehoben werden konnten. Sonst geben wir die nicht seltenen Verirrungen steiler Buchstaben in die schräge Schrift wieder, so: ${ }^{\prime} 16 \mathrm{R}$. $77^{\prime} 38 \mathrm{R}$. $80^{\prime} 11$ R. $97^{\prime} 9$ R. $129^{\prime} 2$ R. $185^{\prime} 41.198^{\prime} 3$ R. $298^{\prime} 19$ R. $370^{\prime}$ Ũ. $386^{\prime}$ Ü. CII'33R. vI'39R. GvI'2R. Versäumt ist das worden bei $\mathrm{E} f a$. $^{\prime} 13^{\prime} 17 \mathrm{R}$. und $54^{\prime} 54 \mathrm{R}$. Sehr häufig kommt das in jenen Theilen des Abc vor die wir dem TB nachgedruckt haben, so Igandea, eta, rom., Iob, Pfal., eph.; vor Allem trifft es hier die Versalien, so Eliça, Taulan u. 8. w., wobei daran zu erinnern dass manchmal in jener Zeit die Schrägschrift durchgehends mit steilen Versalien versehen ist. Das Umgekehrte kommt kaum vor, so ฮ̃ $\mathrm{III}^{\mathrm{b}} \mathrm{b} 10$. Wenn sich im Urdruck zuweilen schräges $G$ statt eines steilen findet (so ${ }^{* * *} \mathrm{III}^{\prime}{ }^{18}$. Abc $\mathrm{KI}^{\mathrm{r}} 10$. L viII ${ }^{\circ} 10.11$ ), so rührt das daher dass letzteres in der entsprechenden Grösse nicht vorhanden war. Die Richtung der Interpunkzionszeichen (beim Punkt kann keine Verschiedenheit sein, beim Beistrich pflegt keine zu sein) wird durch die Schrift innerhalb deren sie auftreten, bestimmt; aber wie unsere Setzer gern nach dem einzelnen schrägen Wort ein schräges Zeichen setzen, so ist das auch denen Hautins häufiger passiert. Der steile Doppelpunkt ist in diesem Falle 2 war das Regelmässige, aber oft steht der schräge, so 47'26. 241'10. $256^{\top} 5-$ und nicht wiederg.: Semeaz: 174 ' 71 , ioaileco: $223^{\prime} 2$, eguitera: $275^{\prime} 19$, Iaun: $291^{\prime} 2$. Der schräge findet sich zuweilen in nur steiler Schrift, so $305^{\prime} 24$. $^{2} 338^{\prime} 1^{\prime}$; häufig weist ihn die kleine Steilschrift der Materientafel auf, so besonders $\sigma r^{\prime} b$. Wenn die Gründe welche die Wahl dieser oder jener Schriftgattung bestimmen, im Ganzen äusserlicher Natur sind, so gilt das nicht für die unter die Steilschrift gemischte Schrägschrift des Haupttextes. Das Studium der durch die letztere hervorgehobenen Wörter, die im griechischen Texte fehlen und deren Hinzufügung dem bessern Verständniss oder einem sprachlichen Erforderniss dient, ist gewiss, besonders wenn man die französische Uebersetzung daneben hält, ein sehr lehrreiches; an dieser Stelle würden wir uns aber nur darum zu kümmern haben was zu viel (z. B. eguin

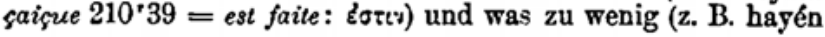




\section{XXII}

artean $2044^{\circ} 19$, hayén artean $204^{\circ} 26=$ au milieu d'eux: cle tò

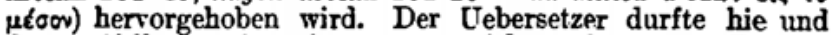
da zweifelhaft sein wie er zu verfahren hatte; z. B. suec

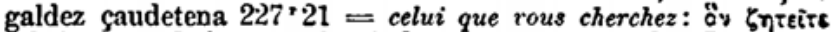
scheint gerechtfertigt sein, indessen ermangelt das Personalpronomen hier durchaus jedes Nachdruckes ("den íhr sucht ${ }^{\mu}$ ) und ist nur aus gewissen formalen Rücksichten hinzugesetzt, ähnlich wie das im Italienischen geschieht. Auf diese Dinge lasse ich mich jedoch nicht näher ein, weil sie zu weit seitwärts von dem vorgezeichneten Wege führen. - In ihrem Schnitt weichen unsere Steilschriften von denen des Urdrucks nicht sehr ab, wohl aber unsere Schrägschriften. Dass in einer und derselben Schrägschrift des Urdrucks ein Buchstabe verschiedene Gestalten annimmt, darauf mache ich aus dem Grunde aufmerksam weil zum Theil auch ein sprachliches Interesse hineinspielt. Es handelt sich nicht etwa, wie in den kurz vorher besprochenen Fällen, um eine irrthümliche Mischung, sondern um eine willkürliche die immerhin durch besondere Anlässe hervorgerufen sein mag. Und zwar findet sie erstens bei den Versalien statt, die entweder nur durch die Richtung sich von denen der Steilschrift unterscheiden, oder die durch ihren gerundeten Körper, die geschweiften Ansätze und Ausläufer kokett hervortreten. Letzteres thun einige nie, nämlich $H, K$, $L, O, s, X, Y, Z$; die übrigen aber stimmen dann im Wesentlichen zu dem "verschnörkelten Kursiv Garamonds (in Verwendung 1640)", wie es bei K. Faulmann Ill. Gesch. der Buchdruckerk. S. 368 abgebildet ist, nur dass das Hautinsche $C$ schmächtig ist und unter die Linie hinabreicht, sein $\varepsilon$ gerundet und 7 (80 im franz. ${ }^{17}$; im TB vielmehr $Z$ ) nicht so viel ist wie $F$, sondern wie $I$. Die Formrerhältnisse ändern sich übrigens mehrfach mit den Schriftgraden; man halte z. B. das $\boldsymbol{X}$ und $A$ des Titelblattes mit dem grösseren $M$ und $A$ der französischen Vorrede zusammen. Doch selbst die gleiche Grösse schliesst Verschiedenheit der Gestalt nicht aus, so 2 ${ }^{*} \mathrm{II}^{\prime} 16$ und $2^{*} \mathrm{VIII}^{\prime} 1$. Die einfachen und die geschweiften Majuskeln folgen nun oft dicht aufeinander; z. B. $\Delta$ : $^{*} \mathrm{IV}^{1} 11$ und $12,{ }^{*} \mathrm{v}^{r} 2$ und $3, G$ : $399^{1} 16 \mathrm{R}$. und 17 R., im franz. ${ }^{17}$ $C: 15^{r}$ Ũ. Wenn man das nicht beachtet, so kann man leicht auf den Gedanken kommen dass man mit dem verlängerten und durchstrichenen $I$ in Iefus, Iofeph, Iaun u. s. w. (84'19. 105'25. '227'36. $244^{\prime} 22$ u. 8. w.) den konsonantischen Laut auszudrücken begonnen babe, für den gewöhnlich noch die einfache Versalie gebraucht worden sei. Ich kann mich 


\section{XXIII}

allerdings nicht entsinnen in unserem Urdruck jenem $J$ mit vokalischer Geltung begegnet $\mathrm{zu}$ sein, wohl aber im franz. ${ }^{77}$ (z. B. in $J l, J_{n}$ 'titutiõ). Z Weitens gibt es unter den Minuskeln eine die sich in doppelter Gestalt zeigt, nämlich $z$, das sich bald emporreckt: $\approx$, bald in die Länge streckt: $\approx$, beide nebeneinander, z. B. am Schluss 209' 24 R., in der Mitte und zwar in demselben Worte: gucizco ${ }^{*} \mathrm{~V}^{r} 4$, litezquen $\mathrm{a}^{\mathrm{r}} 13$ und 14; das erstere Mal hat das $q$ nach dem breiten $z$ seinen Balken eingebüsst. Da die hinabsteigenden Lettern nicht wie die andern an den Oberkörper des $z$ in beiderlei Gestalt, anrücken können, so bildet sich dann jedenfalls ein Abstand wie er zwischen zwei Wörtern einzutreten pflegt: ez gende $\widetilde{a}^{\mathrm{r}}{ }^{\mathrm{r}} 12$ ist sicherlich als zwei Wörter gemeint, aber das $g$ könnte auch in einem und demselben Worte nicht näher am $z$ stehen. Der vierte und stärkste Unterschied innerhalb der Schriften ist der zwischen Majuskeln und Minuskeln. Mit den letzteren zusammen werden immer Majuskeln entsprechender Grösse als Versalien verwendet, und ebenso versehen in der durchgängigen Majuskelschrift oft, nicht immer, grössere Majuskeln die gleiche Funkzion, z. B. 57'37 und in den linkseitigen Kolumnentiteln. Sehr auffällig ist die Abwechselung von CAP. und CAP. auf der rechten Seite von 221 bis 318; jenes erscheint nämlich 221 . 223. 237. 239. 244. 253. 257. 260. 270. 275. 276. 286. 287. 292. 301. 303. 305. 306. 307. 308. 318, weder vorher noch nachher. Es erinnert mich das an den Scherz den sich der Setzer (und ihm der Korrektor) in der soulischen Uebersetzung der Nachahmung Christi (Bayonan, 1883; BB 578) gestattet hat, nämlich von Anfang bis zu Ende als linkseitigen Kolumnentitel JUSU-KRISTEN und JESU-KRISTEN miteinander wechseln zu lassen (jenes $36 \mathrm{Mal}: 4,8,18,22,32,44$ u. s. w.). In Bezug auf die Versalien in der Minuskelschrift sind einige Versehen des Urdrucks zu verzeichnen; kleine Anfangsbuchstaben bei Eigennamen: ${ }^{* *} \mathrm{~V}^{1} 20 . \mathrm{VII}^{*} 11.4^{r} 1$. 314 ${ }^{r}$ 17. $\widetilde{\mathrm{e}} \mathrm{IV}^{1}$ a 8.9 . i $\mathrm{III}^{\prime} \mathrm{b} 41$. $\widetilde{\mathrm{I}^{1}} \mathrm{~b} 17$, zu Beginn eines Satzes: $\mathrm{Dr}^{1} 36$, grosse bei einem Appellativum: Fedez 272' 1 , einem 'Zahlwort: Laurgarren $440^{r} 12$, einem Verb: Euangelizatzen $302^{r} 16$ (dies unter Einfluss von Euangelio, in demselben Vers noch euangeliza), einem Adverb: Frangoqui $245^{\circ} 8$, Halacotz $\tilde{a} \mathrm{v}^{1} \mathrm{a} 21$. In einigen Fällen ist der Gebrauch schwankend, so bei Centener, franz. Centenier (centurio), wenn ein Eigenname dabei steht: Cornelio Centenerari $225^{\prime}$ Ü., Cornelio ..... cẽtener $226^{\circ} 1$, Cornelio centenera 227'22 (franz. ${ }^{63}$ : Cent-, cent-, cent-, "7: -, cent-, Cent-). Capitain scheint auch neben Eigennamen mit $c$ geschrieben $z u$ werden $\left(256^{1} 7,257^{\times} 22\right.$; franz. ${ }^{68}$ : cap., ${ }^{77}$ : 


\section{XXIV}

('ap-), ebenso im Plural, aber mit $C$ im Singular mit bestimmtem Artikel (251'31 u. s. w., nur einmal mit c: $253^{\prime} 27$; hier franz. ${ }^{68}$ : cap., "

Die in unsern baskischen Texten vorkommenden Buchstaben sind dieselben wie die damals für das Französische gebräuchlichen, was im Abc A mI ${ }^{1}$ zur Anschsuung gebracht ist. Wir haben zunächst die 23 Majuskeln des Lateinischen wie sie in den Inschriften auftreten (also auch $K$, aber nicht $J, U, W$ ). Dazu kommt aber noch $G$, welches allerdings eine Modifikazion des mit ihm zum Theil gleichwerthigen $C$ ist und daher von Leiçarraga an jenem Ort nicht mit aufgeführt wird. Dieses $G$ findet sich nicht in allen Schriftarten und wird daher entweder durch das einer andern Schriftart oder, gewöhnlich, durch $C$ ersetzt, 80 bei Inizialen (305'. 344'. 414'), bei Ueberschriften (z. B.

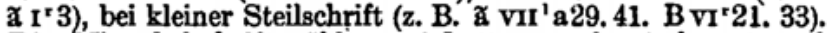
Die Minuskelschrift zählt zwei Lettern mehr, indem $S$ und $V$ differenziert worden sind, jenes in $\int$ und 8 , dieses in $v$ und u. Obwohl deren Gebrauch im Baskischen Leicarragas mit dem gleichzeitigen französischen, italienischen u.s. w. im Wesentlichen übereinstimmt, muss ich ihn doch was den ersteren Buchstaben anlangt in seinen Einzelnheiten verfolgen. Es gilt die allgemeine Regel: im Anfang und in der Mitte der Wörter wird $f$, am Schluss $\&$ geschrieben. Dieses $s$ erscheint aber unter gewissen Bedingungen auch in der Mitte der Wörter. Und zwar sind dies, wenn wir uns zunächst an das Französische halten - und auch Leicarragas Widmung wird uns dafür Belege liefern -, folgende: 1) bei ganz durchsichtigen $\mathrm{Zu}$ sammensetzungen am Ende des ersten Theils, so in der Widm.: treshumble ${ }^{*} \mathrm{I}^{\top} 4$, toutesfois ${ }^{*} \mathrm{II}^{*} 19$ und $\mathrm{II}^{\prime} 9$ neben trefobeiffant ${ }^{*} \mathrm{II}^{\prime} 4$, trefrenommee *II ' 16 , Toutefois * III' 25 , defquelles, toufiours, Tranflateur, pluflost, autreffois; 2) vor $t$ in Schrägschrift und dann mit ihm verbunden (was wir nicht haben wiedergeben können), 80 in der Widm. esté * $\Pi^{*} 10$, ceste ${ }^{*} \mathrm{II}^{*} 13$, teste, feust, est, reste, adiouster neben eflant ${ }^{*} \mathrm{II}^{\mathrm{r}} 9$, efloye ${ }^{*} \mathrm{II}^{1} 3.4$, cefle * $\mathrm{II}^{2} 21$, nofle, Teflament u. 8. W.; ganz ebenso vor $p$; 3) nach $\int$ in Bteilschrift, wenn der folgende Vokal ein Zeichen auf sich hat, so vor $i$ im franz. "s3 (au/si, possible u. 8. w.) und vor Vokal mit Tilde im franz. ${ }^{71}$ (prefenta/sèt, puifsãce u. s. w., doch auch z. B. laiffät 67'52; aber immer auffi, poffible u. s. w., indem dann das $i$ seinen Punkt verliert), und mit f verbunden in Schrägachrift allgemein vor $i$, 80 in der Widm.: außi, meßieurs, poßible. Das Baskische folgt dem Französischen. Auch im ersten Fall ist $\int$ weit gewöhnlicher, doch haben wir deshone $\Omega$ - $307^{\prime} 23.308^{\prime} 5$. $344^{P} 4.414^{\prime} 2\left(^{63}\right.$ : 
dejhon-, deshon-, - deshon-, "1: deshon-, deshon-, -, deshon-) neben defohoratzen $179^{\circ} 49.269^{\prime} 23$ ( ${ }^{63}$ : defhon-, deshon-, ${ }^{71}$ : deshon- beide Male), desfiguratu neben defconfort, defordre, defobedientia, defpleg- u. s. w., transform- 318'18. $327^{\prime} 13.15$ neben trãf-| formatzen $327^{\circ} 14$, tranffigur- tranfport- u. s. w., promesbat D vi'22 neben propofbat B vi ${ }^{\mathrm{r}} 6$. Vor dem Bindestrich scheint $s$ zu herrschen, z. B. bildots-bat $223^{r} 32$, Ioannes-ere $164^{1} 23$, has-erc $\mathrm{E}_{\mathrm{I}^{\circ}} 3$, doch deuf-ere $\mathrm{Abc} \mathrm{B} \mathrm{v}^{\prime} 5$. Baskische Belege für den zweiten Fall gewährt das Abc; im Vorwort finden wir neben dem verbundenen $f t$ in eftimatu, ikaften u. s. w. auch das verbundene st in Christ, irakasteco (wir haben überall unverbundenes $f$ ) und in der Erklärung an den König, die hier in Schrägschrift auftritt ( $\mathrm{K}$ II f.), verbundenes $s p$ in spirituz, fuspirioac. Am häufigsten ist $j s i$ für das immerhin gewöhnlichere $\int f i$ (ohne Punkt auf $\operatorname{dem} i$ ), so crofsi-, hefsi-, $i k u / s i-$, nabu/si-, pofsible u. s. w., in der Schrägschrift $\beta i$ (was wir da wo sie bei uns entfiel - s. oben S. XXI - nicht wiedergeben konnten). Vor abkürzendem Punkt in der Regel $f f$, so coloff., theff., doch in Schrägschrift auch coloß., the $\beta$. (wir mussten $/ \mathcal{s}$ setzen) und ohne Bindung $C_{0} / \rho / s$. (304 $31 \mathrm{R}$.), und so in Steilschrift (z. B. ẽ II ${ }^{1}$ a 10. b 44). Ich gedenke schliesslich des Falles in welchem das Französische sogar im Anfang das $s$ gestattet, nämlich vor dem Apostroph; so in der Widm.: s'est, s'eftoit, s'en neben fe presentoit, fe fervir; ebenso in der Steilschrift von ${ }^{6 \mathbf{s}}$ und ${ }^{7}$, und zwar regelmässig. Der Haken des $\int$ und der Apostroph behinderten einander; man konnte aber auch den letzteren weglassen, so lesen wir im Advertissement Calvins von 1544 (gedr. bei Iehan Girard zu Genf) feft, fen neben c'est, n'en, qu'il. In dem ebenda 1545 gedruckten Katechismus Calvins hat man aber doch Beides miteinander zu vereinigen gewusst: $\int^{\prime} e s t, f$ 'il, $\int^{\prime} e n$ (doch auch fen). Da das Baskische keinen Apostroph kennt, so hat es auch kein solches $s$ '. Ueber $v$ und $u$ ist nichts Besonderes zu bemerken; jenes steht im Anfang, dieses in der Mitte der Wörter sowohl für unser $v$ wie für unser $u$. - Die Buchstabenverbindungen (Ligaturen) sind in unsern Texten ziemlich zahlreich. Leiçarraga zählt deren im Abc neun auf, von denen \& mit seinem französisch-lateinischen Lautwerth für das Baskische ganz entfällt, ct, das wir nicht wiedergeben konnten, hier nur in Fremdwörtern vorkommt (z. B. $4^{18}$ ). Die $f$-Verbindungen ( $f, f, f)$ haben auch wir (nur nicht in der Schrift von $\mathrm{A}$ ff.), nicht aber die vier Verbindungen des $\int$ (mit $i, l, f, t)$, und ebensowenig die von Leiçarraga dort nicht verzeichnete, in der franz. Widmung auftretende mit $p$. Noch 


\section{XXVI}

ist die ron $\int$ mit $s$ in der Schrägschrift hinzuzufügen, die wir wenigstens in der franz. Widmung wiedergeben konnten. Auch 8 wird, wie eben schon erwähnt, mit $t$ und $p$ rerbunden. Unter den "Diphthongen ${ }^{4}$ Leiçarragas befinden sich zwei Buchstabenverbindungen: $a$ und $\alpha$, ron denen die letztere unsern Texten ganz fremd sein dürfte (das griechisch-lateinische oe erscheint hier als e), die enstere aber nur in einer Wortform vorkommt, nämlich in høy und zwar mit monophthongischem Werthe (= e). Nicht verbundenes ae, wie in Ifrael, Nathanael hat eine andere Aussprache. Wenn wir dennoch Haey $156^{1}$ Ü., haei $234^{\prime}$ Ü., haey $359^{\circ}$ Ü. lesen, so beruht das wohl auf dem Umstand dass in dieser Schrägschrift kein $a$ zur Verfügung stand. Dem Wesen nach gehören die Vokale mit Tilde, welche bei $L$. unter den "Abkürzungen" aufgeführt werden, zu den Buchstabenverbindungen; das Tilde ist ein flüchtiges n, das aber auch das $m$ rertritt, z. B. hãbat, ilhũbe (dies löst Vinson Mark. XV, 33 in ilhunbe auf). Als allgemeines Abkürzungszeichen erscheint dieses in Gñatione i II'a31 (Gener.) und Spĩ î IV'a21 (Spiritu). Von den sonstigen an jener Stelle rerzeichneten Abkürzungen sind $9=u s, \tilde{\mathrm{q}}=q u e$ und $q=q u i$ im franz. "ganz gewöhnlich, z. B. $p l 9$, req̃fte. Das erste finden wir ein einziges Mal in unsern Texten: de $9 \mathrm{i}^{\circ} \mathrm{b} 18$, und ebenso ein einziges Mal die Abkürzung für pro- $248^{1} 28$.

Wenn $f$ und $s$, sowie (in L.s Augen) $v$ und $u$ nur verschiedene Gestalten eines Buchstaben sind, so begegnen uns nun auch Buchstaben die als wirklich verschiedene gelten, (und zum Theil auch Buchstabenverbindungen) mit dem gleichen Lautwerth unter verschiedenen äussern Bedingungen. Die fremden Wörter, vor Allem die Namen spielen hier wie überhaupt eine besondere Rolle, indem sie sich dem baskischen Laut- und Schriftsystem nicht anzupassen pflegen. $Y$, welches in L.s Alphabet enthalten ist, bedeutet nichts Anderes als $i$, tritt aber in echtbaskischen Wörtern nur als zweiter Theil eines Diphthongen auf und zwar wiederum nur und das regelmässig am Ende des Wortes oder innerhalb des Wortes vor einem Vokal, ganz im Einklang mit dem damals herrschenden französischen Gebrauch, also bay, etfay, etfayac, gayo neben baina, etfaigoa, sait - guiçoney, hodey, hodeyetan neben ceilan, cembeit, deithu, wie franz. fay, ayant, foy, royons neben faire, voir. Nur ist othoi das Regelmässige: * ${ }^{*} I^{\prime} 23$. A I $^{1} 9$. II ${ }^{\prime} 18$. 35 u. s. w. Abc A vII'1. 12. 27 u. s. w., und ebenso dei: $6^{\prime} 21.42^{\prime} 9$. $44^{\prime} 10$ u. s. w. Vor $h$ besteht ein gerrisses Schwanken: 
XXVII

eihartzen $193^{\prime} 6$.

leikorra $44^{\prime} 15$, -rrean $73^{r} 47$. eyhar $21^{\prime} 10.40$ '19. 20. 64'1. feihets Bv'6, -t/a 202'34. $204{ }^{\prime} 20.230^{\prime} 7,-t$ ean 204 '27.

Wider die Regel ist $y$ gesetzt in: boeytabat $51^{\mathrm{T}} \mathrm{T}$. sayt $324^{1} 1$. ctfaytaffun $406^{*} 4$. gaynean $38^{\prime} 25$.

inşayqueon $165^{\prime} 10$. natzayçue $293^{1} 1$.

Seynaleren DII'27, seygnale seignale $53^{1} 48$ u. 8. w. EII'26.

Und $i$ in:

baieta ${ }^{*} \mathrm{v}^{1} 25$. Abc Brir6. bayeta ${ }^{*} \mathrm{vI}^{\mathrm{r}} 31$. '27. Abc $v^{\prime} 13$.

banarreii 351 '12.

batheiatu '291'16, -atzen batheya-4'6.'11.13.14 u.s.w. F III $^{1} 36$ R.

ceguei $\gamma \mathrm{n}^{\mathrm{r}} \mathrm{b} 28$.

eiçue 88'9. $248^{\prime} 28$.

heri 41 ' 36 , haei 234 ' Ü.

maneiojo $405^{1} 17$.

moien- D I'38. '17 R. u. 8.w. moyenic D IV'36, moyen EI ${ }^{1} 34$. Sogar $y$ im Anlaut für $i$ als Konsonant:

yeraguc ẽ II'b 3 . yetzaguc A III' $23 \mathrm{R}$.

ieģaguc $139^{\prime} \tilde{5}$.

ietraguc A vi ${ }^{1} 28$.

In den griechischen Wörtern bleibt $y$, so my/lerio, Pumphylia; wird nur ganz ausnahmsweise durch $i$ vertreten:

Eutiche $247 \cdot$ Ü.

Lideaco 223 ' Û.

Falsch steht $y$ für $i$ :

Hyerapolen $358^{1} 13$.

hypocryfia $321^{\prime} 6$.
Eutyche 247 '9.

Lyddan 225'32. 35, -a '38. (franz. ": Lydde, Lidde, Lydde).

hypocrifia $129^{r} 1$, -az $45^{1} 28$. $370^{1} \cdot 2$.

$-U$ hat $\mathrm{L}$. in einem einzigen baskischen Wort durch das dem Französischen entnommene ou ersetzt, nämlich in haour "Kind", um es von dem gleichlautenden haur "dieser" in der Schrift zu scheiden. Ein par Mal ist Beides miteinander verwechselt worden : 


\section{XXVIII}

haour ** VII'21. 351'15. haur. $371^{\prime} 4$ (es geht in demselben Vers haourric voran). Brv'13.

haur 422 ' 1 .

haour.

Auch in romanischen Wörtern pflegt L. ou nur ganz ausnahmsweise zu schreiben, so pourpr- 450 个. $452^{\prime} 12$. 16 und:

recours $\mathrm{EVI}^{1} 16$.

recurfa $\mathrm{EII}^{2} 27$.

Der Laut $[s]$ wird, mehr nach dem Muster des Spanischen als dem des Französischen, durch drei Buchstaben wiedergegeben: $c$ (vor $e, i), s$ ( Konsonanten und - das ist dem Baskischen eigen - nach $t$ : etzen, etzuten). Auch vor dem bestimmten Artikel und dem Fragewort $-a$ bleibt $z$ (voza, eza), aber doch:

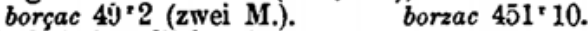

Noch befremdlicher ist:

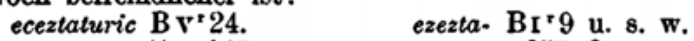

Ebenso selten kommt das Umgekehrte vor:

bazaizquio EII'13.

berze II 'T' b 31 .

ezagutzen 294' 20 , -uturic

A $\mathbf{I}^{1} 15$.

guizonéc 164 ' 19.

$Z$ wird in fremden Namen und Wörtern, wie Zacharias, zelo schlechtweg übernommen, hat aber dann eigentlich den Werth eines stimmhaften [s]. Wir finden es auch gemäss dem franz. 62 und 71 in:

Elizabeth Mm IV'a $4 . \quad$ Elifabeth $97^{\prime} 5$ u. 8. w. è vir'a 13.

Genezaretheco ã $\mathrm{IV}^{*} \mathrm{~b} 20 . \quad$ Genefaret( $h$ )co $28 \cdot 34 . \quad 73^{\circ} 53$. $108^{\mathrm{r}} 1$.

$\zeta$ erscheint einige Male für $c$ vor $e$ :

geineten $415^{r} 12$.

geraucan $68^{\prime} 10$.

serbait F vuI' 13.

ciegen $80^{1} 11$.

citeçen $32^{1} 6$.

susenéz ã I' $\mathrm{I}_{27} 2$.

egeinere $\mathrm{F}$ vIII ${ }^{3} 3$.

egen 22 ' 36 .

- Mit dieser Gleichwerthgruppe $c, \varepsilon, z$ verknüpft sich durch das doppelwerthige $c$ die andere, ebenfalls dem Romanischen entnommene $c$ (vor $a, o, u$, Kons. und am Wortschluss) und $q u$ (vor 


\section{$\operatorname{XxIx}$}

$e$, $i$ ) für den Laut $[k]$. Abgesehen von fremden Namen und Wörtern (so Quart 290'23), schreibt L. in einem einzigen Falle qu anstatt $c$ vor dunklem Vokal, nämlich in baiquaitu $100^{\prime} 78 \mathrm{u}$. s. w. und in allen entsprechenden Formen mit ai von $v k a n$, edin, içan, wie baiquaitugue, baiquaitezque, baiquaitzaque, baiquaizquio - niemals, wenn ich Nichts übersehen habe, baicaitu u. 8. w., wie zu erwarten wäre. Vielleicht ist das mit Rücksicht auf die Niedernavarrer geschehen, für die ja auch die Schriften L.s bestimmt waren; diese nämlich sagen buiquitu, freilich auch guitu. Wir können auch annehmen, in L.s eigener Sprache habe die Form baiqueitu gelautet - wir begegnen bei ihm ähnlichen Dissimilazionen (s. unten S. XLVIII) -, und er nur das ai von gaitu auch hier in der Schrift erhalten wollen. So würde es sich erklären dass wir sonst gai-, nicht guai- geschrieben finden, oder dies nur ganz ausnahmsweise: guaizquion Abc $\mathrm{H} \mathrm{VIII}^{2} 17$.

Anderseits findet sich auch einmal ezquaitzaitza (Evir ${ }^{138}$ ) für ezgai-. In baicara und in allen entsprechenden Formen mit a (z. B. baicaquidizquio Abc $\mathrm{BI}^{\prime} 19$. $\mathrm{rV}^{\prime} 5$ ) bleibt immer $c$; ein $\mathrm{Mal}$ habe ich qu gefunden:

buiquarate Abc A vil' 22 ,

wo wohl soul. baiquirate (so 400'28) vorschwebte. Dem $c$ und $q u$ gesellt sich in Fremdwörtern als drittes Zeichen $c h$ (in baskischen Wörtern $=$ vord. $[\tilde{s}]$ ) zu, wofür dann und wann die heimische Schreibung eintritt, so:

Corazin $20^{\circ} 21$.

Criften $348^{\prime} 19$.

Cuzen กิ $\mathrm{IV}^{1} \mathrm{~b}+3$. Chorazini $19^{\circ} \ddot{\mathrm{U}}$., -zin $123^{1} 13$.

Ariftarque 244 'Ü., -quec Chuz 116'3. $247^{\prime} 4$. Ariflarche 246 ' 29.

Dem $c$ und $q u=[k]$ steht zur Seite die Gruppe $g$ (vor $a, o, u$, Kons.) und $g u$ (ror $e, i)=[g]$, die mit der zugehörigen $g$ (vor $e$, i), ge, $i$ (ror $a, o, u)=[\bar{z}]$ (gende, iugeatzen) wiederum vom Romanischen geliefert worden ist. Doch eignet die letztere nur romanischen Wörtern, und der Laut selbst ist ein unbaskischer, der daher wohl schon damals in der gesprochenen Sprache eine grössere oder geringere Anpassung erfahren haben wird. - Die aspirierten Tenues welche heutzutage da wo sie noch gesprochen werden, die gleichförmigen Bezeichnungen $k h$, $p h$, th haben, finden sich bei Leiçarraga $k$, $p h$ (im Anf.) und $p p$ (zwischen Vok.), th geschrieben, bei Dechepare $q h$ (auch $c c), p h$ und $p p h$, th, bei Axular $k h$ und $c c, p h$ und $p p$, th; Voltoire (1642; BB 12b) schreibt für das erste c-h. Ganz ausnahmsweise hat auch L. $k h$ : 


\section{XXX}

khen $40^{1} 21$.

Wie befremdlich auch

daccufjagun $\mathrm{B}$ III ${ }^{\mathrm{r}} 1$.

daccarraçuen $193^{\prime} 8$.

daccarquescue $199^{1} 29$ ken $42^{\prime} 13$; u. s. w.

dacuffagun 95'36.

sein mögen, es kann sich doch nicht un $c c$ für $k$ handeln, da wie unten ersichtlich sein wird, die aspirierte Tenuis diesen Formen fremd ist. Vielleicht übte die Schreibung der Fremdwörter hier einen Einfluss aus, in denen $c c$ dasselbe bedeutet wie $c$, z. B. accordaturic $230^{\prime} 11$, accommettitu $258^{\prime} 11$. - Ph ist aber daneben in den Fremdwörtern mit dem Werthe ron $f$ herübergenommen worden. $F$ ist eigentlich ein unbaskischer Laut, begegnet uns aber in einigen haskischen Wörten deren fremder Ursprung ganz verwischt ist, wie affari. $P h$ für $f$ ein $\mathrm{Mal}$ in : prophanatu 251 '28. profanatzen $256^{\prime} 6$; u. s. w.

In Fremdwörtern hat th keinen andern Werth als $t$; daher gelegentlich beide, rerwechselt werden :

Thychique $358^{\circ} \mathrm{U}$.

Tychique 358' 7 .

- Gedehnte und rerdoppelte Konsonanten kennt die baskische Sprache nicht; wo daher in echtbaskischen Wörtern Doppelkonsonanten geschrieben sind, bezeichnen diese grösstentheils andere Laute als die entsprechenden einfachen Zeichen. Dies ist der Fall bei dem erwähnten $p p$, sowie bei $r r$ und $l$ (nach $i$ ), die mit ihren romanischen Werthen eingeführt sind. Dass man damals zwischen Vokalen $f f$ schrieb (ikuffi, heute ihkusi) ist ebenfalls den Romanen nachgemacht worden, aber ohne im Baskischen irgend welchen $Z$ weck zu erfüllen; denn wenn im Französischen und im Altspanischen neben dem stimmlosen $\iint$ das stimmhafte $\int$ steht, so kennt das Baskische den letzteren Laut nicht. Sogar :

Aitaf-| faindu G vi'21. Aitafaindutaffun G'v'3:.

Weshalb Leiçarraga immer herffi schreibt (Dechejare herfi), sieht man nicht ein; schwebte ihm etwa die sonst bezeugte Form hert $f i$ vor? Vereinzelt steht auch das regelmässige ff von affari (Dechepare afari). Endlich hat man aus etymologischen Rücksichten die Doppelkonsonanten gewahrt; doch war selten Gelegenheit dazu. Man begegnet auch der Vereinfachung:

bataffunaren $\mathbf{G} \mathbf{v I I}^{r} 3$. baltaffuna $208^{2}$ U., -un G III 32 . ceinetarico $368^{\prime} 15(=-i c+c o)$.

Auf offenbarem Versehen beruhen die doppelten Konsonanten in : additzea $\mathrm{BII}^{1} 24$. aggueri $435^{\circ} 18$.

Cenchrecco $290^{\prime}$ Schl. (o.f.Cenchreeco nach fr. Cenchree?).

Cenchreco 289'1 (vgl. Cenchren $\left.244^{\circ} 18\right)$. 


\section{XXXI}

erraitten $30^{\prime} 2$.

leinnuac $37 \times 28$.

Ebenso dreifaches $r$ und $\int$ in:

Berriz 2:1' 1 R.

ikuijßric B II'31.

In Fremdwörtern pflegen die Doppelkonsonanten beibehalten zu werden, und so wird in appacega $268^{\prime} 31$, apparaillua $262^{\prime} 19$ das $p p$ in romanischem Sinne $=[p]$, nicht im baskischen $=[p h]$ gelten. Doch in letzterem gerriss das von appaincen "bereiten "schmücken" $41^{2} 2^{\prime} 5$ u. s. W., obwohl auch dies romanischen Ursprungs ist (span. apañar, kat. apanyar ,herrichten", „bereiten “, nausbessern" ${ }^{\mu}$, schmücken $\left.{ }^{\mu}\right)$. Es kommt jedoch daneben nicht selten, zum Theil wie in der fremden Sprache selbst, der einfache Konsonant ror: acomettuturic $126^{12} 2 \mathrm{f}$. flaca $30^{\circ} 32$, -aturen $75^{\prime} 3 . \quad$ flaccataffun $*^{* *} \mathrm{~V}^{1} 23$, -nac $287^{\prime} 1$, flaccuac ebd. 29 , -ago C viri ${ }^{1} 30$, flacquesá ** VIII 19 .

Iaques $5^{\prime}$ Ü. 6r21. (so regel- Iacques 17 r 2 ; u. s. w. mässig im fr. ${ }^{68}$ und ${ }^{77}$ ). permetitzen $369^{\prime} 12$. prome-|seguin A I' 35 . quitatzen CVII' 33. raportatu ${ }^{* *} \mathrm{III}^{1} 32$. refuscita 17'8, -atu 2'2'24. $277^{\circ} 11.281^{\circ} 9$, -aturen 297 '14.

fuportatu * VIII'33, -aturè $33 \cdot 1 \%$, fuporturequin *'vi' 28 . tyraniatic D II' 19. permettitu 36 r8; u. s. w. promeffén $A \mathrm{II}^{\prime} \overline{5}$; u. s. w. quitta $\mathrm{Abc} \mathrm{BIV}^{\prime} 4$; u. s. w. rapportari $267^{\prime} 30$.

reffufcitatzen $260^{\circ} 8$ (im franz. ${ }_{68}$ und " regelmässig reff.).

.upportatu 287'1; u. 8. พ. Tyrãno $245^{\prime} 9$.

Beispiele des $\mathrm{C}_{\text {mgekehrten sind : }}$ sppollos $243^{\circ} \mathrm{C}$., $-o c \mathrm{i} \mathrm{IV}^{-1} \mathrm{a} 30$. Paullen '25\%' $\mathbf{C}$. guittarrarién $446^{1} 2$, rrác $448^{\prime} 2$.

epheff. i II 'b 47 . III $^{1}$ a 44.

Apollos $244^{1} \mathrm{~W} 4$; u. 8. w. Prulen $257^{\prime}$ Ü.; u. s. w. guitarrac $446^{1} 2$.

Ephcs. $46^{1} 4$ R.; u. s. w.

Der letztere Fall befremdet um so weniger als einerseits bei der Abkürzung $\int f$ öfter durch $f$ vertreten wird (Colof. Thef.; rgl. Philip. Pier.) und anderseits die Neigung besteht $f$, wie in baskischen, so auch in Fremdwörtern doppelt zu schreiben; so regelnässig pauffu '20'29 u. s. w., pauffaturen $123^{\prime} 6$, ferner ieloffi $326^{\prime} \cdot 2.336^{\circ} 17$. - Hier sind nun alle jene Varianten der Lautbezeichnung anzuschliessen welche die aus den Ro- 


\section{XXXII}

manischen entlehnten Wörter im Romanischen selbst schon aufweisen; der Zwiespalt zwischen etymologischer und phonetischer Schreibung setzt sich bis ins Baskische fort. So haben wir $c t$ neben $t$ :

appoinctamèdua $421^{\prime 2}$. ẽ $\mathrm{I}^{\prime}$ appointadi $7^{\prime} 24$.

b $9,-\iota m e n d u \quad 424^{\prime} 10$, -atzeco ẽ $\mathrm{I}^{1} \mathrm{~b} 11$.

licteretan $215^{\prime} 15$.

fuiect $285^{\prime} 5$.

Ferner $c i$ neben $t i$ :

tentacionetan $126^{\circ} 4$.

audiencián '259 23 .

fuiet $103^{\prime} 51.285+1$ u. 8. w., fuiette ${ }^{*} \mathrm{vI}^{*} 31$.

tentationetan $9^{1} 13$; u. 8. w.

Gewöhnlich nämlich behalten die schon in der lateinischen

Litteratur belegten und sonst unveränderten Wörter ihr $t i$, also abundantia, patientia, gratia u. 8. w. Aber regelmässig wird doch precio geschrieben. Vgl. ferner:

viciofoa A Iv'24.

vitiofoa F III'6; u. s. w.

Umgekehrt dringt ein latinisierendes $t i$ in romanische Wortfornien ein :

foberantiataco Abc Brv'12. foberancia 324'1, -atacoz 3699. trifletián ${ }^{* *} \mathrm{vIII}^{\prime} 9$.

Bei L. ist allerdings die gewöhnliche Form triftitic (z. B. $195^{\circ} 6$ ); aber wie finecia $3433^{14}$, paubrecia $323^{\prime} 2$, so sagte man auch damals schon tristecia (heute finecia, tristecia) neben flaquesa (so auch heute) u. a. - Von denjenigen Schreibverschiedenheiten welche uns nicht in demselben Wort aufstossen (z. B. compainia - Efpaignia - jeignoria; veilla - beilhaqueriazco F vIIr '21) schweige ich. Nur bemerke ich dass wenn das sog. stumme $e$ im Auslaut französischer Wörter, das ja, nicht bloss in Südfrankreich, im 16. Jbrh. noch lautbar zu sein pllegte, im Baskischen meist wie das bask. $e$ behandelt wird (digneac, iugeac, princeac; in dem letzten Wort geht es sogar vor $e$ in $i$ über: princiéc $38^{1} 25$, princiey $71^{1} 21$, princietaric $293^{\prime} 8$ entgegen reguéc, echetarat u. 8. w.), das doch wohl nicht immer der Fall ist, wenigstens heisst es zwar Pilote, aber Pilati, Pilatgana.

Die bisher vorgebrachten Varianten haben das Gemeinsame dass sie keinen Unterschied der Aussprache ergeben, wenn auch damit nicht gesagt sein soll dass sie alle wirklich auf einem Schwanken in der Rechtschreibung beruhen. Ich wende mich nun zu der zweiten weit grösseren Gruppe der Varianten, derer mit welchen ein Unterschied der Aussprache verbunden ist. Sie würden eigentlich wieder in zwei Unter- 


\section{XXXIII}

abtheilungen zerfallen, die der Varianten welche der Sprache L.s oder dem Baskischen überhaupt nicht zugeschrieben werden können, die also den Charakter mechanischer Druckfehler tragen, und die der Varianten welche als gesprochene Formen entweder nachweisbar oder doch denkbar sind. Aber da es hierbei doch ohne Unsicherheit und demzufolge ohne Willkür nicht abgehen könnte, so ziehe ich es vor die ganze Masse nach äusserlichen Gesichtspunkten $\mathrm{zu}$ ordnen, und wo mir Lautwandel vorzuliegen scheint, das ausdrücklich zu bemerken. Manches freilich was man vielleicht hier erwartet, wird erst später beim Morphologischen und Syntaktischen zur Sprache kommen; denn auch in dieser Richtung sind die Grenzen zum Theil unsicher.

1) Verwechselung zwischen einzelnen Buchstaben (auch z. Th. zwischen einem solchen und einer einen einfachen Laut ausdrückenden Buchstabenverbindung):

a) zwischen Konsonanten oder zwischen Konsonanten und Vokalen. Zum grössten Theil wird sie auf die Aehnlichkeit der Typen zurückzuführen sein; doch wirken noch andere Anlässe, z. B. wird an die Stelle des erforderlichen Buchstaben leicht einer von denen gesetzt die sich in seiner unmittelbaren Nachbarschaft befinden. $a$ für $n$ :

complimeadua ì viI' $\mathrm{b} 35$ $(a$,$) .$

$b$ für $d$ :

beabruarenác** $\mathrm{VI}^{\mathrm{r}} 3\left(b_{1}\right)$. $b$ für $h$ :

babilationea $\mathrm{D} \mathrm{VI}^{\mathrm{i}} 18\left(b_{1}\right)$. bambat DyI'27 $\left(b_{1}\right)$.

bebar $51^{\prime}$ U. $\left(b_{2}\right)$.

beb. I II' a $15\left(b_{1}\right)$.

epbes. $375 \cdot 9 \mathrm{R}$.

b für $p$ :

nolazbaita B II' 30 .

$b$ für $u(=v)$ :

aburtoin $311^{\prime} 8$ (südfranz.). auortoimbat ê $\mathrm{II}^{*} \mathrm{~b} 5$.

$c$ für $s$ :

caretencát $418^{12}\left(c_{1}, c_{2}\right)$.

cayola CIV'2.

cayoneà ** $\mathrm{II}^{*} 39$.

ciecán $147^{1} 30$.

ciecón $97^{\top} 13.99^{\prime} 34$. 


\section{XXXIV}

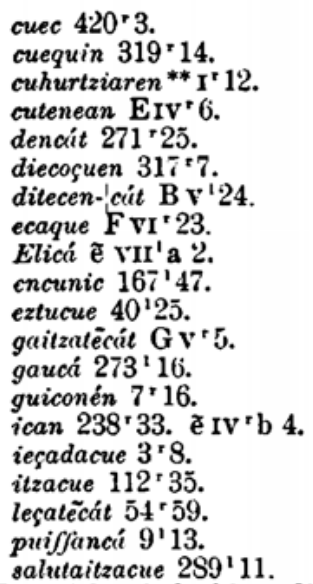

Natürlich sind hier diejenigen Fälle nicht bemerkt wo die betreffende Schrift kein $s$ besitzt (s. S. XXIV).

$$
\boldsymbol{c}=\boldsymbol{e}:
$$

conucrtitzera A $5 \cdot 13\left(c_{2}\right)$.

erideitcn ** VIII' 9 .

Iudaca $257^{1} 24$.

Es sollte stehen Iudu; ich kann nir die Form nur erklären wenn sie unmittelbar aus dem lateinischen Texte herübergenommen worden ist, wie Samaritana $165^{1}$ 7. 9, obwohl auch dann Iudea zu erwarten gewesen wäre.

$$
c \text { für } g \text { : }
$$

carcagarritaco $\mathrm{Gr}^{1} 7\left(c_{2}\right)$.

corputz $51^{1} 12$ (Einwirkung

des lat. corpus).

Amorecatic kann nicht hierher gezogen werden; es ist die regelmässige Schreibung $\left(81^{\prime} 29.351^{\prime} 7 . \mathrm{av}^{\prime} \mathrm{a}^{\prime} 23\right.$. B $\mathrm{rr}^{\prime} 10$. Crv'28. DI'18. IV'5; mit $g$ ist es mir gar nicht aufgestossen), auch das Niedernavarrische und Soulische sagen so (amorecatic schon bei Dechepare und in den westniedernav. Pregariac von 1651 [BB 23d] S. 2. 17. 22). Ich vermag dieses $r$ nicht zu erklären, da sonst überall nach Vokal wie Konsonant -gatic geschrieben und gesprochen wird; Salaberrs (BB 277) bemerkt S. 10: "Le mot propre serait amoregatik; par corruption on a substitué la lettre $k$ à celle $g . "$ 


\title{
$\operatorname{XXXV}$
}

\author{
c für $k$ : \\ rcan $303^{\circ} 5$ (unmittelbar vor \\ Iaincoac).
}

L. schreibt rkan, rkaiten, aber eduqui, eduquiten. In demselben Verbalstamme wechselt regelmäвsig $k$ mit $c$, nach Massgabe der Lautumgebung, oder wie wir vermuthen dürfen, der Betonung. So haben wir $i k u f f i$, ikuflen, ikujJac, ikufquir,ue, aber dacuffute, badiacufquiat, becuf/a, und eracutfi, eracuften; ikaffi, ika/len, aber iracat/i, iracaflen; ekarri, ekarten, ekarroçue, ekardaşue, ekatzue, aber sacarquela, dacazquet, ezpaitacarque; jedoch statt des zu erwartenden erac- tritt uns erekarri, erekarten entgegen, sogar ezterakarran $299^{\circ} 18$, also $k$ nach $a$. Aehnlich, aber nicht ganz übereinstimmend ist das Verhältniss $z$ wischen th und $t$ bei ethorri, ethorten, athor, dathorrenean, banathor, bethor - satozte, datırquela, baniatorquec.

$$
c \text { für } o \text { : }
$$

ençutecc è vilI' a $4\left(c_{2}\right)$.

jac A vi' 29 .

$$
c \text { für } r \text { : }
$$

c für $t$ :

ceruetarac $\mathrm{C} \mathrm{rI}^{\circ} 7 \mathrm{R} .\left(c_{\text {, }}\right)$.

$\boldsymbol{s}$ für $c$ :

çaiçu ** ${ }^{1}{ }^{1} 34\left(\varsigma_{2}\right)$.

cerauşan CVI'9.

communiçatzeco $426^{\prime} \mathrm{C}$.

drauşa DVI'29.

edifiçatu $386^{\circ} 3$.

gucias eे $\mathrm{IV}+\mathrm{l}$ 14.

Iainşoa $10^{1} \cdot 24$.

marudiçatu ${ }^{* *} \mathrm{VI}^{\mathrm{r}} 2$.

s, c für ch:

cekenqui 324'6 (zwei M.). chekengui ẽ vIII' a 22 (soul. chekena à viI' a 19).

sucen $4^{\prime} 3$, -na 2:24'11. chuchen- $100^{\prime} 79$ u. s. w.

$358 \cdot 1.418 \cdot 15$.

gaigo $\mathrm{B}$ VII ${ }^{1} 35$.

Eigentlich ist $c h$ für $\&$ verkleinernd; aber vielfach ist der Unterschied aufgehoben, und die sekundäre Form die herrschende.

$$
\underset{\text { tsftimonio für } e:}{* \mathrm{IV}^{-1} 2 \mathrm{~S} .}
$$




\section{$\operatorname{XXXVI}$}

s für $h$ :

be-|sarAbc A viri 29 (die vorhergehende Zeile beginnt mit şa).

$$
\begin{aligned}
& \text { s für (t)s: } \\
& \text { fantajiagru GV'24. vIII'5 } \\
& \text { (vgl. -azco } \mathrm{G} \mathrm{II}^{1} \text { 21). } \\
& \text { harricuetara } 23^{\prime} 5 \text {. 24'20. } \\
& 66 \text { '5.' } 16 .
\end{aligned}
$$

ithumbeģu $449^{1} 10$.

mẽdiçu $100 \cdot 65$.

Dieses - gut ist niedernavarrisch für lab. $t$ fiu (harritfu, menditfu, odolfu; bei L. verthutetfu, vrgulut/u, iaquinful).

$$
\text { ch für s: }
$$

charqui 403'2.

şar $15^{\prime} 16$; u. 8. w.

Wie eben gesagt, ist $c h$ eigentlich verkleinernd.

$d$ für $c, c$ :

$$
\text { daicula Cv'17. ģaicula. }
$$

Das werde ich unten, im Zusammenhang mit andern Verbalformen, zu erklären versuchen.

$$
\begin{aligned}
& \text { deçaqueagu } \mathrm{CVIII}^{1} 16 \text { (mdl.). cegaqueagu } \mathrm{C} \text { viI }{ }^{1} 35 . \\
& \text { eztitiát } 434^{\circ} 2 \text { (ditiát; mdl.). citiat } 49^{1} 20 \text {, citiagu u. в. w. } \\
& \text { d für th: } \\
& \text { aurditē }{ }^{* *} I^{1} 22 \text {. } \\
& \text { aurthiteco } 179^{\prime} 59 \text {, -iten } 406^{\circ} 5 \text {. }
\end{aligned}
$$

An der $\mathbf{z w e i t e n}$ Stelle ist die Bedeutung des Verbs: ${ }_{n}$ werfen ${ }^{4}$, wofür sber die Wörterbücher vielmehr aurthiqui, arthiqui und auch mit $d$ aurdiqui, aurdigui angeben; und so hat auch L. 73.48 aurthiquilen (franz. $\dot{a}$ tirer $=d$ tirer $\dot{a}$ la rame). An der ersten Stelle übersetzt nola bere arraga maradicaturat aurditè baitute guciéc das franz. Sentans tous leur maudite race, und an der dritten Inuidiatara aurthiten du: conuoite $\dot{a}$ enuie, und wir sehen deutlich dass das baskische Verb welches dem rom. tirer, tirar "werfen" entspricht, ihm nun auch in die übertragene Bedeutung: „(in eine Farbe) fallen “, „(einer Sache) ähneln", „(auf Etwas) hinstreben“ folgt.

$e$ für $c$ :

diaerenac ẽ VII ${ }^{1} \mathrm{~b} 6\left(e_{1}\right)$.

ieenac $^{* * *} \mathrm{IV}^{\prime} 2\left(e_{1}\right)$.

Lue. ${ }^{* *} \mathrm{IV}^{2} 16 \mathrm{R}$.

lue. i $\mathrm{rv}^{\mathrm{r}} \mathrm{b} 48$.

Der zweite Fall ist nicht wiedergegeben, weil zuerst $c$ gelesen wurde; in der That handelt es sich in diesem und im 
vierten Fall (hier aber nur im L., im St. ist ein deutliches c) um schadhafte e.Typen.

$$
f \text { für } \boldsymbol{s}:
$$

menofpreciatu ${ }^{* *} \mathrm{VI}^{1} 15$.

$g$ für $k$ :

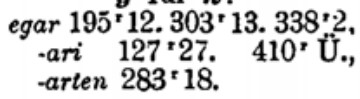

Es ist das sicherlich dasselbe Verb wie ekarri, von dem es sich hier in der Bedeutung durchaus nicht unterscheidet; ja es folgen $33^{\mathrm{r}}$ nahe aufeinander: elkarren cargác egar ilzaçue und bere cargá ekarriren du, und dem gure bekatuac ekarri $411^{\prime} 24$ entspricht das gure bekatuac egari der Kapitelüberschrift. Es ist egari eigentlich eine mundartliche Form von ekarri, die aber zum Theil für die gleichen Mundarten mit etwas verschiedener Bedeutung verzeichnet wird; bei Larramendi egartu unter "llevar", ecarri unter "traer", bei Gèze egari als "pré: Berver, garantir" und "supporter", ekharri als "apporter", bei van Eys egari lab. als "user", ekarri als "porter, apporter, amener". Dechepare BII'8 hat die vermittelnde Form egarrico, mit doppeltem $r$, wodurch freilich Verwechselung mit egarri „durstig“ (ebd. CIr²0) hervorgerufen wird.

\section{$g$ für $p$ :}

gorgutza i $\mathrm{II}^{\mathrm{*}} \mathrm{b} 39(\mathrm{~g})$.

$g$ für $q$ :

c., chekengui è IV'a 14. $15 . \quad$ cekenqui $324^{\prime} 6$. VIII'a 22.

nenguiçuencát $265^{\star} 20$, nen- guẽquinztén $239^{1} 13$. guién $328 \times 33$.

In beiden Fällen warnt die Wiederholung vor der Annahme einer Buchstabenverwechselung; der erstere ist wohl dem lab. ungui a soul. hounqui zu vergleichen, im letzteren mögen Formen von eguin sich eingemischt haben.

$g$ für $r$ :

bothege $\mathrm{Cr}^{\prime} 2 \mathrm{R}$. (es folgt unmittelbar gucitaco).

$h$ für $b$ :

gahe ${ }^{* *}$ VII'27.

heh i $\mathrm{I}^{\mathrm{r}} \mathrm{b} 21\left(h_{2}\right)$.

hetheric $212^{1} 8\left(h_{1}\right)$.

ilhühea $429^{1} 13\left(h_{2}\right)$. 


\section{XXXVIII}

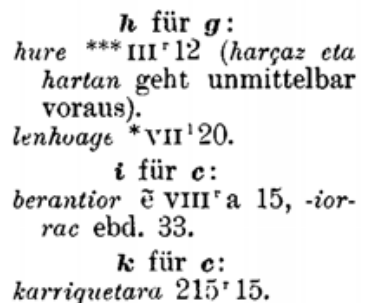

korduc $263^{\circ} 32$, -atoz $162^{1} 15$.

carrica $9^{\prime} 5$, -iquetan 21'19.

$133^{r} 26,-$ iquetara $123^{\prime} 10$. $135 \times 21$.

L. pflegt das $c$ im Beginn der romanischen Wörter zu wahren, im Inlaut $k$ für $c c$ und $c$ (nach Konsonanten) nur in ganz eingebürgerten Wörtern (bekatu, barkatu) zu setzen.

1. $\mathrm{Fv}^{+} 4$.

$\boldsymbol{m}$ für $\boldsymbol{h}$ :

$m$ für $n$ :

ceim i IV $^{1}$ a 46 (ilhumbe steht unmittelbar darüber).

Chanaam Mm III'b 21.

Iaincoarem $\mathrm{C}_{\text {III }}{ }^{1} 8$ (es folgt

Semea).

mincenetic $256^{\prime} 11$.

In den drei ersten Fällen kann man an eine falsche Auflösung des Tilde denken ebenso wie in:

$\boldsymbol{n}$ für $\boldsymbol{m}$ :

cenbat $126 \cdot 8$.

$\boldsymbol{n}$ fïr $\boldsymbol{u}$ :

Anri Kal. II ${ }^{2} 2$.

gniaizquió 217 '4.

Auri Kal. II ${ }^{1} 2$.

lnc i VIII'b 48.

$p$ für $g$ :

porputz Abc B III'25.

$\boldsymbol{q}$ für $\boldsymbol{g}$ :
equiteco $358^{17}$.

ezquitzaizte $332^{1} 5$.

ezquaitzaitza $\mathrm{EvII}^{1} 38$.

Nach ez- werden $b$ und $d$ verhärtet (nach bai- auch $c$ : baicara), aber ezgara, ezgaituzte u. s. w. 
$[\boldsymbol{r}$ für $n$ :

belhaurico $221^{\mathrm{r}} 60.225^{\mathrm{I}} 40$. belhaun $286^{1} 11.349^{1} 10.399^{1} 12$, $249^{\circ} 36$. $^{1} 5$, -rica- $33^{\circ} 14$. -netara $108^{\prime} 8$, nic $282^{\circ} 4$, $56^{1} 29 . \quad 62^{\circ} 40 . \quad 81^{\circ} 17$. -nac $342^{1} 14$.

$94^{\circ} 19.152^{1} 41$.

Van Eys hat, wie man sieht, Unrecht kurzweg zu sagen, L. schreibe belhaur für belhaun. Jenes ist allerdings eine Form die einer oder der andern Md. (so dem Südhochnavarrischen) eignet; hier aber kommt sie nur vor -ic + Vok. zur Anwendung (vgl. alt- und neusüdfranz. dimergue, morgue u. s. w.).] $\boldsymbol{r}$ für $\boldsymbol{t}$ :

Cejarearic $257^{\prime} 1\left(r_{2}\right)$. crearura $320^{\prime} 17\left(r_{2}\right)$. era ẽ VIII'b 34 .

handiraffun FVIII ${ }^{*}$ 39, -na ebd. ${ }^{1} 8$.

Eine mundartliche Form; S. Pouvreau (BB 788) gebraucht ebenfalls neben dem gewöhnlichen handitafun zuweilen handirefun, so S. 27.52 . 56 .

hilrzeracoun $\tilde{\mathrm{e}} \mathrm{VIII}^{\mathrm{r}} \mathrm{b} 32\left(r_{1}\right)$.

gorpurz i I II a $1\left(r_{2}\right)$.

Abgesehen von der Nachbarschaft eines $r$ in fast allen Fällen, ist die grosse Aehnlichkeit zu bedenken welche in diesen Schriften zwischen $r$ und $t$ besteht.

$$
\boldsymbol{f} \text { für } \boldsymbol{b} \text { : }
$$

fu/mettitu 332' 5, -itzeco submettitzen $\mathrm{GV}^{1} 29$ (lat.).

F VIr ${ }^{1} 1$ (bearn.).

$\boldsymbol{f}$ für $\boldsymbol{c}$ :

A/fenfionetico C vi ${ }^{\mathrm{r}} 12 \mathrm{R} .\left(\mathcal{S}_{2}\right)$.

f für ch:
firmendu $264^{r} 3$.

chirmendu 193'2, -uac 193'4.

5 , $-u a 193^{1} 6$. $\widetilde{\mathrm{e}} \mathrm{IV}^{\mathrm{r}} \mathrm{b} 45$.

Es verhält sich damit wie mit $\xi$ und $c h$ (s. oben). $f, s$ für $f$ :

dif-| famatzera $\mathrm{D}$ VII ${ }^{1} 26$, dis- diffamatzen $\mathrm{D}$ VII ${ }^{1} 17$. fumagale 377\%2 (span.). $f$ für $l$ :

Legiffatora A virI $8\left(f_{2}\right)$. $f, s$ für $z$ :

ikus garria $\mathrm{F} \mathrm{II}^{1} 23 \mathrm{R}$. ikuzgarria $\mathrm{FrI}^{1} 22$.

Es ist zugleich Wortverwechselung; ikusgarria bedeutet „das Sehenswerthe".

laburfqui $\mathrm{B} \mathrm{II}^{\mathrm{r}} 29$. VIII'28.

Zwar kann ich laburzqui, die im Labourdischen und Nieder- 
navarrischen herrschende Form (= soul. llabursqui) bei $\mathrm{L}$. nicht nachweisen, aber er hat arinzqui ${ }^{* *} \mathrm{II}^{r} 14$, communzqui $\mathrm{BvII}^{1} 30$, bardinzqui $\mathrm{FV}^{\prime} 15$, wo allerdings kein $r$, sondern ein $n$ dem $z$ vorhergeht. Neben laburfqui steht das Adverb der gewöhnlicheren Bildung, laburqui, so $415^{\circ} 12$. Erv'2. Abc H VIrI'22.

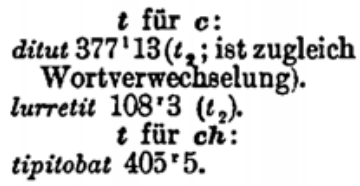

Hier hat $t$ offenbar den Werth des mouillierten $t$, der später durch $t$ ausgedrückt wurde, und es liegt eine mundartliche Variante vor. Auch das to ist so viel wie tto und wechselt mit -tcho:

haourto- 191'33. 336'19 haourtcho. 3'8. 11. 13 u. s. w. u. 8. w. („Kindlein" im („Kindlein" im eig. S.). geistlichen $\mathrm{S}$.).

Zum grössten Theil aber vertheilen sich die beiden Formen der Deminutivendung auf verschiedene Wörter: appurto, emazteto, iflato, kordato, açauto (25'30; 8. van Eys Dict.) - choritcho, gambratcho, liburutcho, ohetcho, lempletcho.

$t$ für $d:$

albeitzinteitze 111'23; vgl. cindeiztençát $316^{1} 4 . \quad 323^{1} 9$. cintezquete 193'28.

$$
327 \times 7 \text {. }
$$

L. zieht hier das $d$ vor, obwohl dadurch der Einklang mit guentecen, guentezque, guintezque und weiter mit guentuen, guintuen aufgehoben wird. Das Guip. hat guindecen, cindezten; das Lab. und Soul. guint, cint-; für die franz.-bask. Mdd. im Allg. verzeichnet Dartayet (BB 319 c) guintecen, guindecen und cintezten, cindezten, aber Salaberry gibt in der That für das Niedernav. an: ginten, cindezten.

chuchent (ceiten) 132'11.

(cedin) 13. (itzaçue) $399^{\prime} 12$.

Vgl.unten S. LII ilhund u. s. w.

$$
t \text { für } h \text { : }
$$

baitaiz $13^{1} 19.122^{1} 57$.

baihau $8^{1} 41$, baihincén $205^{1} 18$; u. s. w.

Van Eys VAL S. 30 Anm. 2 deutet die Möglichkeit an dass auch in baiheģaquete - $t$ - stände; das ginge wegen der $\mathrm{Zweideutig-}$ keit nicht (vgL. baiheşaquegu, „dich" Abc BII 25 , baiteşaquegu ,ihn“ Abc B III'19). 


\section{$\boldsymbol{t}$ für $\boldsymbol{i}:$}

antmalac $435^{\prime} \mathrm{T}$.

$\boldsymbol{t}$ für $\boldsymbol{r}$ :

arthatfean $215^{121}$ (statt arra-; Einmischung von arthat/fu).

ethot $\mathrm{DI}^{1} 27\left(t_{2}\right)$.

itubaizte i v $\mathrm{v} 36\left(t_{1}\right)$.

hartoca $11^{\mathrm{r}} \mathrm{U}$.

hertoquétan $438^{\circ} 15$.

arroca $12^{r} 24$ (harrocaren $\mathrm{TB}{ }^{28}$ ebend.).

Vgl. harroqui bei Pouvreau, worin van Eys harri-toqui erblickt. natutaren $\mathrm{A} \mathrm{IV}^{\mathrm{r}} 23\left(t_{2}\right)$.

othoit DIv'30 $1 t_{1} ;$ wir

haben aus Versehen den

Druckfehler verbessert).

Vgl. oben „r für $t^{4}$. So ptompt im franz. ${ }^{77} 304^{r} 2$, echetean in den guip.-bizc. Sprichw. von 1596 (BB 6-7) 10, 15, otoençat ebend. 30, 11 (umgek€hrt beratu für betatu ebend. 12, 5).

th für $d$ :

eritheitzebat Gv'35, „Ein-

bildung" oder "Erfin-

dung:", wenn es auf erideiten zurückgehen sollte.

beranthuren $396^{\circ} 37$.

minthuric $260^{\circ} 11$.

$\boldsymbol{\imath}$ für $\boldsymbol{n}$ :

berandua 72'35 (zwei M.).83'11. karmindu $440^{\circ} 11$, -uraz $270^{\prime} 14$, famindura $344^{1} 31$.

Spirituau ẽ $\operatorname{VIII}^{\mathrm{r}} \mathrm{a} 25\left(u_{2}\right)$.

$\boldsymbol{u}$ für $\boldsymbol{y}$ :

cauán 112:30.

cauenac $387^{\prime} 6$.

Van Eys VAL S. 47 Anm. 2 meint, das letztere (cauenenac ist Druckfehler) stünde für cerauenac. Aber ganz abgeschen davon dass es sich dann weder als eigentlicher Druckfehler noch als mundartliche Variante leicht erklären liesse, entspricht sayenac dem Wortlaut des Urtextes wie dem der franz. Uebersetzung (aufquels il a efté annoncé), während die andere Lesung bedeuten würde: ,denen er es verkündet hatte"; auch vergleiche man zu diesem denũtiatu içan çauenac: denuntiatu içan etzayenéc $288^{\prime} 21$. Ebenso zu der Stelle der ersteren von van Eys nicht erwähnten Form: Efcatzen çaún guciari emóc: 


\section{XLII}

Efcatzen gayanari emóc $8^{1} 42$. Ich werde diese Verbalformen unten, im Zusammenhang mit andern, zu erklären versuchen.

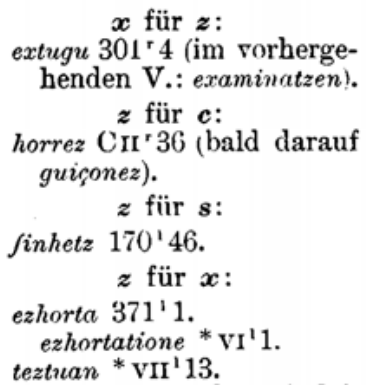

Ein Interpunkzionszeichen für einen Buchstaben:

:ro tara A VII ${ }^{\mathrm{r}} 11 . \quad=$ orotara.

Ein Buchstabe für eine Ziffer:

$r 7$ o $\mathrm{IV}^{\prime} \mathrm{b} 31$.

$i$ 80ㄱ R. (i. cor.; nicht wie- $=1$.

dergegeben). $141^{\mathrm{r}} 26 \mathrm{R}$.

$141^{\mathrm{P}}$ I R. $142^{\prime} 14$ R.

b) zwischen Vokalen. Diese Verwechselungen sind im Verbältniss weit häufiger. Die leichte Vertauschbarkeit welche \%wischen allen vokalischen Lauten besteht, erzeugt nicht nur eine Menge wirklich mundartlicher Varianten, sondern auch individueller und gelegentlicher. Wie die Vokale gern versprochen und verlesen werden, ebenso gern werden sie auch verschrieben und verdruckt. Insbesondere spielt die Vorausnahme eines zu sprechenden, die Wiederholung eines gesprochenen Vokals eine bedeutende Rolle. Setzer denen die Sprache fremd ist, werrlen sich etwas anders benehmen als Setzer deren Muttersprache sie ist; sichere Rückschlüsse auf die Nazionalität der Setzer werden sich aber aus den Drucken selbst kaum ziehen lassen. In unserem Falle möchte ich allerdings baskische Setzer vermuthen, einestheils weil ich es für misslich halte alle mundartlichen Varianten auf Rechnung Leiçarragas zu ketzen, anderseits weil hie und da, wovon schon Belege gegeben 
sind, ganze Wortformen, freilich in sinnloser Weise, miteinander vermengt zu sein scheinen.

$a$ für $e$ :

accufatzan 257 r $21\left(a_{\mathrm{g}}\right)$.

interrogatzan $195 \overline{5}\left(a_{2}\right)$.

amplega Arv'18 $\left(a_{1}\right.$; franz.

Ausspr.).

arrachqui $\tilde{\mathrm{a}} \mathrm{VII}{ }^{\circ} \mathrm{b} 32\left(a_{1}\right)$.

appartenitzen ${ }^{*}$ vinr 23 .

$344^{1} 3$. G viII 13 (franz.).

aznaguian $332^{1} 2\left(a_{1}\right)$.

baldur $372^{1} 20$.

bira $130^{1} 35$ ( $\sim$ gara, dira [de]).

berahala $257^{1} 26 . \quad 262^{1} 17$

$\left(a_{1} ;+\right.$ bera $)$.

caretala $354^{\circ} 10\left(a_{2}\right)$.

cembaitrabeit $257^{1} 24\left(a_{2}\right)$.

daclaratzen CVIII'20 $\left(a_{1}\right)$.

daftaturan $179^{\prime} 52\left(a_{\mathbf{3}} ;+\right.$ -aturán).

emparadoregoaren $\tilde{\mathrm{a}} \mathrm{v}^{\mathrm{r}} \mathrm{b} 34$ $\left(a_{1}\right)$.

ereitara $66^{\circ} 3\left(a_{1} ;+\right.$-tara $)$. vkaitara $\mathrm{E} \mathrm{VI}^{\mathrm{r}} 32$.

erra (badadi) 294'15 (und immer erratzen).

ezpaguina $326^{\circ} 14 . \mathrm{Fv}^{1} 35$ (mdl.).

ezterakarran 299: 18 .

fidal $303^{\mathrm{r}} 13$ (es folgt $d a$; oder $\sim$-al \} franz. -el?). gaituala $\mathrm{F}$ VI ${ }^{\circ} 17\left(a_{2}\right)$. yarauzquic A VI ${ }^{1} 18$. gendetzecoataric $175^{\prime} 31$. hemandic 193' 31 .

hunadrano $153^{\circ} 51.154^{\circ} 5$ (wohl für hunat-drano).

itzaradoqui $\mathrm{D} \mathrm{II}^{1} 23$.

erre (citzaten, cedin) $246^{\circ} 19$. $440^{\circ} 7$ (auch erre içaten und immer erreren).

baguine Evi' 33 .

erekarrac $378^{1} 11$; u. s. w.

guerauzcac $436^{1} 10$.

gendetzecoetaric $176^{\circ} 40$.

hunedrano Kal. $\mathrm{II}^{1} 27.28$ (neben oraindrano, noizdrano, egunerano; vgl. huneraino in andern Mdd.).

itzeradoqui $\mathrm{DvI}^{\mathrm{r}} 18 \mathrm{vIH}^{1} 34$.

E Ir ${ }^{12}$. Abc I v' 30 (nverpflichtet $\left.{ }^{\prime}\right)$. 


\section{XLIV}

mafacatzen $449^{\prime} 10(a$, ; ich kann keine andere Form dieses Verbs nachweisen).

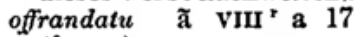
(franz.).

faluamand $u$ i v'a $9 \quad\left(a_{3}\right.$; franz. Ausspr.).

fargeantari $7^{1} 25,-\operatorname{tac} 240^{1} 35$ (südfranz.).

foffagu $14^{-2} 26.117^{\prime} \cdot 24 . \mathrm{Gv}^{2} 7$ (gleich darauf foffegatzen).

offrendatzeco ãiv'b17; u. s. w.

sergeantéc $240^{1} 38$.

joffegurequin $367 \cdot 12$, jofsega $28^{\circ} 32.73^{\circ} 51$; u. s. w.

Dieses foffagu ist jedenfalls mundartlich: Larramendi führt sosagatu, sosagua neben den Formen mit $e$ an; Aizquíbel ebenfalls sosagua und sosagutu, Beides aus Leiçarraga, letzteres mit der Zahl LXXIII (wo foffega steht), gemäss der grossen Liederlichkeit mit der dieses Wörterbuch herausgegeben worden ist (wie ja auch sosagutu in sosagatu zu verbessern ist), Fabre sosagîa unter "tranquille". Das $a$ scheint im Romanischen nicht begründet zu sein (span. sosegar, sosiego, kat. sossegar, sossego). - Neben daiteque findet sich in den bask. Mdd. auch daitaque und besonders ditaque, wohl in Folge von Dissimilazion; dieses $a$ ist auch $\mathrm{L}$. nicht fremd, ich habe es wenigstens ein Mal angemerkt:

citaqueen $213 \times 14$.

Vgl. dazu satequeen $262^{\prime} 21.387^{\prime} 8 . \mathrm{F} \mathrm{v}^{-1} 31$; dataque, sataqueen u. $\varepsilon$. $w$. scheinen auch die heutigen Mdd. nicht zu kennen. Endlich hat L. sehr bäufig an Stelle des $e$ als Vorvokals des Präteritums das eigentlich den Präsens zukommende a (über diese und die umgekehrte Vertretung 8. meine Bask. Stud. I, 5. 24 ff.). So (ich stelle die einzelnen Wurzeln voran): Lşa trans.] ģaşaten $201^{\circ} 15$, laşaten cegaten $64^{1} 6$ u. s. w., le[daf] Başadaffaten $90 \cdot 5$.

[di] andin $367^{1} 3$. ezladin $185^{1} 37.390^{\prime} 11$. ezlaite $47^{\prime} \cdot 22$, bailaite ** IV' 32 .

[gi+di] saidianean $277 \cdot 3$, naidiqueen ${ }^{*} \mathrm{VI}^{1} 18$, laidi leidioten 111'11. $181^{1} 33$, laiditenac $263^{\prime} 43$.

[quitdi] baitzaquidizquion $62^{1} 4 . \quad$ cequidizquion $117^{1} 19$. ezlaquidigu $\mathrm{F}^{1} 36 . \quad$ lequidie $\mathrm{F} \mathrm{mI}^{1} 31$. 


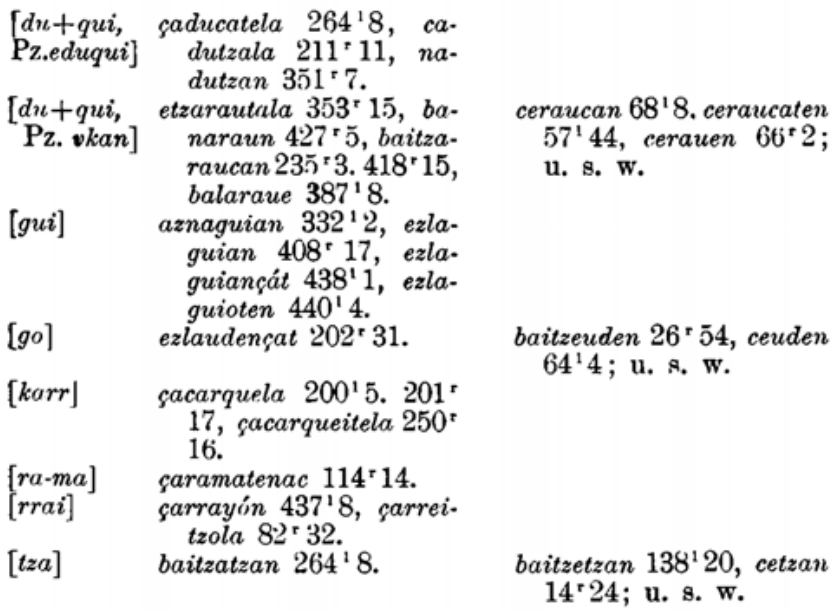

Ich habe hier auch diejenigen Fälle eingereiht in denen bei L. das $a$ im Präteritum das Gewöhnlichere, vielleicht das Alleinige ist, (ez- scheint dissimilierend $-a$ - nach sich zu begünstigen; besonders lehrreich ist $390^{\prime} 11$ ). S. auch unter $e$ für $a^{u}$ (S. XLVII). Und dahin habe ich auch die Vertauschungen zwischen $e i$ und $a i$ verwiesen.

$$
a \text { für } o \text { : }
$$

Dahatfu $402^{\prime} 12\left(a_{1}\right)$.

$$
\begin{aligned}
& \text { guiçana i } \mathrm{VIII}^{1} \mathrm{a} 11^{1}\left(a_{1}\right) \text {. } \\
& \text { halacara }{ }^{*} \mathrm{~V}^{1} 22\left(a_{\mathrm{s}}\right) . \\
& \text { iayat egunaz îIII'a } 16 \text { (von } \\
& \text { iayo; vgl. lab. sortegun } \\
& \text { neben sor-egun). } \\
& \text { Salamon }{ }^{* *} \text { vIII'2 (allg.). }
\end{aligned}
$$$$
\text { Salomon } 1^{1} 6 \text { u. s. w. }
$$

Mit dem -0- der dativischen 3 . P. wechselt bei L. vielfach $-a \cdot$; man kann nicht sagen dass das Eine älter ist als das Andere, beide sind aus -au- entstanden. So:

gaizcan $46^{1} 1$.

gaquitzan $\mathrm{B} \mathrm{rv}^{1} 23$.

drauca, draucat, draucagu

u. s. w. durchweg. saizconetaric $161^{\prime} 40$. gaquitzon çat ${ }^{* *} \mathrm{VI}^{1} 28$. draucoala $\mathrm{B} \mathrm{II}^{\top} 3$, oan $\mathrm{C} \mathrm{VII}^{1} 16$, -oán Abc B VI ${ }^{\mathrm{r}} 16$, diraucoé $377^{\prime} 8$ (also -0 - vor Vokal). 


\section{XLVI}

daritzanac 285'8 (ebd. daritzocuen), -ana 421 × 10 . daritzagu $425^{\mathrm{r}} 19$, -agunean $425^{1} \cdot 2$.

daritzaten 347 г 24 .

çarreitzate $90^{\mp} 13$.

diotfa, ate, ciotfa, ate (-ala, -atela) $33^{1} 26$ u. s. w.

$\boldsymbol{a}$ für $\boldsymbol{u}$ :

larambate $\mathfrak{a} \mathrm{vI}^{\mathrm{r}} \mathrm{a} 11\left(a_{2}\right)$. miracaluric $\mathrm{GI}^{1} 26\left(a_{2}\right)$. sainda $412^{r} 5$ (es folgt Iaincoa).

au für o :

rcpaufa- $220^{1} 49 .{ }^{* *} \mathrm{III}^{\mathrm{T}} 17$ u. s. w., repaus Abc $\mathrm{BIV}^{1} 10$.

Das au ist hier zugleich ursprünglich und südfranzösisch; nur letzteres ist es in: compaufa- $\tilde{a}^{1}$ a 14 . Abc Irv ${ }^{*}$ 18. VII $^{1} 10$.

difpaufa. $\tilde{\mathrm{a}} \mathrm{v}^{1} \mathrm{~b} 29 . \mathrm{CI}^{12} 2$. E rV ${ }^{r} 25 . \mathrm{G} \mathrm{II}^{\mathrm{r}} 28$. III $^{\mathrm{r}} 38$.

Abc BII ${ }^{7}$. $\mathrm{IV}^{1} 16 . \mathrm{III}^{1} 1$. III $^{\mathrm{r}} 18$.

e für $a$ :

aitzineratu $\mathrm{G}$ vir 8 .

geineten $415^{\mathrm{r}} 12\left(\epsilon_{\mathrm{s}}\right)$.

ceinetera $373^{\prime} 12$

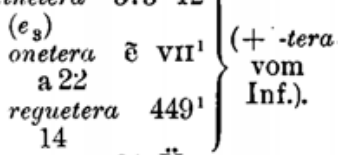

contretaco $64^{\text {r }}$ Uे. u. s. w. crefpe $454^{r} 8$ (franz.). deçadençat $172^{1} 38\left(e_{2}\right)$. daritzón $346^{\circ} 33$.

daritzogun $423^{1} 23.427^{\circ}$ ว.

daritzoteney $278^{\circ} 27.293^{\circ} 9$. carreitzola $82^{\mathrm{r}} 32$.

diotfó u. s. w. $\overline{0}^{1} 6$ u. s. w.

(zu Anfang gebraucht; unterliegt dann nach einem kurzen Ringen den -a-Formen). repofa- $72 \times 31$ u. s. w. 
Elies $7^{\prime} 4$ (wegen franz. Elias $19^{\prime} 14$ u. s. w. Elie).

elegrancarequin $* * I^{*} 31\left(e_{1}\right)$.

elkerri $190 \times 14\left(e_{2}\right)$.

ergnia $47^{1} 29$ (nach bere).

iregan $33^{\circ}: 20$ (ebend. iraganen).

perticipatzen $301^{\prime} 13\left(e_{1}\right)$.

repporta $240^{1} 38$ (südfranz.). The ffulonicerat $378^{\prime} 10$. v/egetacotz $376^{1} 21\left(e_{1}\right)$.

rapportari $266^{-1} 30$.

Theffalonicura $241^{\circ} 1$. reagetun $\left(i \mathrm{~V}^{\mathrm{r}} 12\right.$.

Für präsentisches $a$ findet sich bei $L$. einige Male das dem Präteritum entstammende $e$, no:

ezteçagut $55^{r} 72.74$, baiteşa. nuçague $161^{1} 48$, badaçagut (guz'quic 208 r24.

grercuzcac $436^{\prime} 10$. $179^{1} 55 ;$ u. s. w. garanzcac $436^{\prime} 9$, garanzquic nequionģat $333^{\prime} 19$. A vi' 18. daquionçát $161^{\circ} 31$.

In dem Präsens von ein paar Verben, wie deza, dema hat sich schon bei L. das $e$ ganz festgesetzt; immerhin finden wir:

diaçagun 52 17 (VAL S.25 dieçaçuedãçát 266'11; u. s. w. Anm. wird das Fehlen dieser Formen "le à toi" behauptet).

aģan $196^{\circ} 30$, aģanean baheg,a $8^{1} 39$, ezergadan $256^{\circ} 4$. $134^{1} 8$.

Auch derra scheint gewöhnlicher zu sein als darra:

badarracue $39^{1} 3$, eztarradan ezterraten $381^{1} 2$, ezterrala $383^{\prime} 19$. $402^{1} 13$; und sonst.

$E i$ für $a i$ ist besonders zu behandeln. Es findet sich bei L. zunächst in einigen Formen die als mundartliche Varianten zu betrachten sind, so :

beithan $419^{\circ} 8$.

ceguei $\widetilde{1} \mathrm{I}^{\mathrm{r}} \mathrm{b} 28$, ceguey $\mathrm{E}$ cegay ã I ${ }^{\mathrm{b}} \mathrm{2} 29$. VII ${ }^{r} 10$.

ceitadan $276^{\circ} 10$.

ceyin $265^{\mathrm{r}} 22$ (Praes.,

nicht Impf., wie VAL

S. 34 steht).

balitzeigrue $413^{\prime} 1$.

gueizqui 61 ' 32.

çaitadala 276 r 10 .

heineco Kal. ${ }^{1} 4$.

ezpalitzaic 201.11, balitzuicu D $\mathrm{III}^{\mathrm{r}} 27$. 


\section{XLVIII}

Da L. -guey und -gay („Stoff zu ....") braucht, so wird wohl

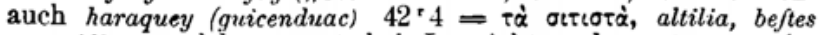
engraiffees, welches sonst bei L. nicht vorkommt, zu guip arakai, arkai "gesalzenes Fleisch" zu stellen sein. Nebenbei gesagt, befremdet es dass ein davon abgeleitetes Verb folgt: haraqueitatu dirade $=\tau \varepsilon$ Sัu $\mu \dot{v} \alpha$, occisa sunt, font tuees. Mehrfach begegnen wir bei $L$. einem durch den vorhergehenden Vokal bedingten Wechsel zwischen ai und $e i$ im gleichen Stamm. So heisst es, in Folge von Dissimilazion, regelmässig iarreiqui, darreit, garreitza u. s. w.; nur ausnahmsweise:

narrayó 351 '14.

garrayón $437^{\prime} 8$.

garraitzala B VIII 31 .

Wo aber das a der ersten Silbe dem $e$ weicht, bleibt in der folgenden das ursprüngliche ai, so berrait $31^{1} 24.77^{\circ} 34$. 121 '23. Ebenso wird wenn dem bai- der Verbalformen alvorhergeht, und zwar nur dann, das ai zu ei: albeitindoa, albeiledi, ezalbeilequi u. s.w. Gegen diese Regel verstossen und zwar in verschiedenem Sinne:

ezpeitarreicu $79^{1} 38$.

albaitzindezte $119^{1} 4$.

Unter diesen Umständen befremdet die Folge ai-ai in: buitaidigute $\mathrm{G}_{\mathrm{II}}{ }^{\prime} 8$. - Auf andern Ursachen, und zwar solchen die ich nicht zu erkennen vermag, beruht bei L. der Wechsel zwischen $a i$ und $e i$ in dem -bait der unbestimmten Fürwörter und der daran sich anschliessenden Umstandswörter. Anderswo besteht kein Unterschied: norbait, cerbait, cembait, nolazpait, noizpait, nonbait. L. schreibt norbeit, cembeit und behält in den übrigen $a i$ bei. Gegen die Regel verstossen:

norbaitec $119^{\circ} 46$.

cerbeit ${ }^{* *} \operatorname{III}^{1} 4$.

Ausserdem hat er -beit in cembaterebeit $229 \times 13$, cembatrebeit $239^{1} 12$, cembaitrabeit $257^{1} 24$.

$\boldsymbol{e}$ für $\boldsymbol{i}$ :

ahãce $403^{\mathrm{r}} 24$. ahãci $399^{\circ} 5$.

Hierzu ist zu bemerken dass L. regelmässig ohortze (139ri2.

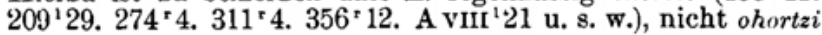
hat.

aitzetic FIV ${ }^{1}$ o.

dacazquet $339^{\circ} 17$ (oder ist es die Futurform dacazquiquet?). 


\section{XLIX}

defeguenic $232^{1} 19\left(e_{3}\right)$.

enflamma E II ${ }^{1} 20$.

inflammatu $\mathrm{E} \mathrm{II}^{1} 36$.

In den Imperfekten mit der 1 . oder 2. $\mathrm{P}$ als Subjekt (auch des passiven Verbs, also mit dem Objekt des transitivaktiven) wechseln als Vorsilbe -en- und -in- miteinander; die einen Formen bevorzugen dieses, die andern jenes. So:

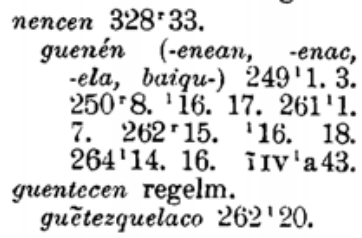

guendoacela $239^{1} 16$, -cençát $333^{\circ} 9$.

nèdutenean, bainendutén $265^{\circ} 18, \quad$ nëduqueitenic

* vI' 21 .

guentuela $260^{\circ} 13$, baiquentuen $262^{1} 20$, guentuztén $399 \times 10$.

centuzten $350^{1} 26$. nincen regelm.

guinen $335^{\circ 23}$, ezquinén $262^{\prime} 21 ;$ u. s. w.

ezguintezque $306^{\circ} 31$. cindeiztençát $316^{i} 4.323^{\prime} 9$. $327 \times 7$.

baininduen $331^{1} 15$, banindusue $191^{\prime} 7$, ninduqueçue $178^{\prime} 42$.

baguintuc A VI ${ }^{\mathrm{r}} 11$, guintuen A vi' 12 .

bacintuztét $288 \times 15$, etzintuqueiztedan $329^{1} 20$.

Auch in den Imperfekten mit der 1. oder 2. P. als Urheber (also mit dem Subjekt des transitiv-aktiven Verbs):

baguendu Abc A viI ${ }^{\mathbf{r}} 12$.

guẽtuenean $250^{\mathrm{r}} 12$.

cenduten $79^{\circ} 33$, etzendu.

tenean $335^{1} 8$, baitzen-

duten $350^{\prime} 26$, bacen.

dutén $352^{1} 10$.

centuzten $76^{1} 19.20$. $335^{1} 8$.

guendrauçuen $361^{1} 4$, ezpaiquendrauen $237^{1} 24$, cendrauzquedetela $336^{\mathbf{r}} 15$. baguindu FI'39.

guintuen A vI ${ }^{\mathrm{r}} 12$.

cindutençát $316 \times 15$, etzindutén $352^{1} 10$, bacindute $357^{\prime} 22$.

cintuzten $31^{\circ} 9.10$.

ezquindrauqueán $199^{1} 30$.

Das - $i$ - welches mit zur Kennzeichnung der Dutzformen dient, geht vor $a$ nicht selten in $e$ über, so:

baceaquiat $61^{\mathrm{r}} 24$, etzeaquie baniaquián $186^{\circ} 42$, ciaquié $155^{1} 34 ;$ u. s. w. 199 r21. 
L

ezteacu/jagu A v'28.

gaitzeaiztec A v ${ }^{1} 21$.

guinetidiquec $\mathrm{Av}^{\mathrm{r}} 30$.

badiacuffagu A vir 4 .

ciaiztec $372^{1} 24$.

$i$ für $a$ :

accommettitu $258^{\mathrm{r}} 11 . \quad$ acomettaturic $127 \times 22$.

Vgl. promettut", permettitu (span. prometer, permitir).

baiquirate $400^{\circ} 28$ (soul.). garate $363^{\circ} 17$. nitziayec $76^{\prime} 24\left({ }^{*}\right.$ natziayec

+ mdl. nitzayec).

$i$ für $e$ :

Arimathiatic $58^{\circ} 57$ (alt- Arimathea- $95^{\prime} 43$ u. s. w.

überlieferte Nebenform).

bihinere $104^{1} 17\left(i_{1}\right.$; bihia bere steht fast unmittelbar darüber).

dathorrinẽ̃ $131^{\circ} 43$.

hiriac $197 \mathrm{r} 9$.

mendicaturic $\mathrm{E} \mathrm{vi} 28$ (lat.).

permittitzen $\mathrm{CII}^{r} 9$ (lat.).

S. ,e für $i^{\prime \prime}$.

dathorrenean $130^{1} 37$.

hireac, -éć $197^{\circ} 6.10$. mêdeca $219^{r} 24$; u. s. w.

permetti $14^{\text {r }} 21$; u. s. w.

\section{$i$ für $u$ :}

ecagutiren $66^{\prime} 13$.

erait/ia $273^{5} 5,-t / i \quad 381^{1} 6$, erautfiren $209^{\prime} 17.18,-t / i$ -t: $446^{\circ} 13$, Eraitfagne $210^{\circ} 33.228^{\circ} 45$. $162^{1} 8$.

Es ist dies das Faktitiv von iautfi, das in andern Mdd. als jachi, jatzi (jaizten, jaisten) auftritt.

\section{$o$ für $a$ :}

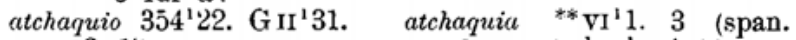
III 3 . $1 \%$.

achaque + bask. Art.).

Vgl. amorio, bozcario, laudorio, mendequio (Genefioa Gir24), in neueren Büchern defendio u. a. Auch schreibt L. regelmässig blafphemio, wohl \} spätlat. blasphemium.

dogocan $\mathrm{Bv}^{\mathrm{r}} 4\left(o_{1}\right)$.

Egyptionoéc Kal. VI' $19\left(o_{1}\right)$.

Notth. $129^{1} 11 \mathrm{R}$.

$\mathrm{S}$. „ $a$ für $o^{\prime \prime}$.

o für $e$ :

abyfimora 118 31 (span.). abyfmera $281^{\circ} 7$ und abyfme-

ejcatzon $406^{r} 3$.

überhaupt. ota $31^{1} 24 \mathrm{R}$. 
$o$ für $\boldsymbol{u}$ :

abondatu $273^{1} 15$, abondo- abund- $7^{1} 20$ u. s. w.

Jago ì II 'a 18 (franz.).

egoitetaric $248^{\mathrm{r}} 19$ (Ver-

wechselung mit egoiten).

topinaguile $55^{1} 7$.

$\boldsymbol{u}$ für $\boldsymbol{a}$ :

tupinaguile $55^{1} 10$, -leac $280^{r}-21$

(südfranz. toupino).

Irucafteco ĩ v'a 38 (kursives $u$ ).

$\boldsymbol{u}$ für $e$ :

fermutate $374^{\circ} 17$ (vgl. fer- fermetute $419^{1} 17$ u. s. w. mutajfiun).

guun|sanşut $\mathrm{CrV}^{\mathrm{r}} 23 \mathrm{R} .\left(u_{2}\right)$.

tiratzun $205^{\prime} 8$ (es folgt sutela).

$\boldsymbol{u}$ für $\boldsymbol{o}$ :

aburtoin 311'8 (südfranz.). Auortoimbat è II ${ }^{*} \mathrm{~b}$ う̄.

Apoftulu B VIII' $32\left(u_{1}\right)$.

gogueta $226^{-1} 19$.

onduan $306^{\circ} 2 . \mathrm{Ev}^{\mathrm{i}} 35$.

Vgl. unten S. LIII -au-, -eu- für -ago, -ego-.

c) Vertauschung ganzer Silben:

ça für ce:

içan CvI'4 (es steht içan

zwei Mal in der vorher-

gehenden Zeile).

ce für ça:

icen $383^{\circ} 7$.

Wären diese beiden Fälle lautlicher Natur, so würde es sich nur um je ein Element handeln.

ta für hi:

betango i viII ${ }^{\mathrm{a}} \mathrm{a} 43$.

behingo $395^{r} 10$.

2) Fehlen von Zeichen:

a) von Konsonanten und von Vokalen zwischen Konsonanten:

$-\boldsymbol{a}$ :

leçn Cvir11 (an).

Das $a(c)$ zwischen dem pronominalen Anlaut $d$ oder $g$ und dem $r$ des Verbalstamms fällt leicht aus: 


\section{III}

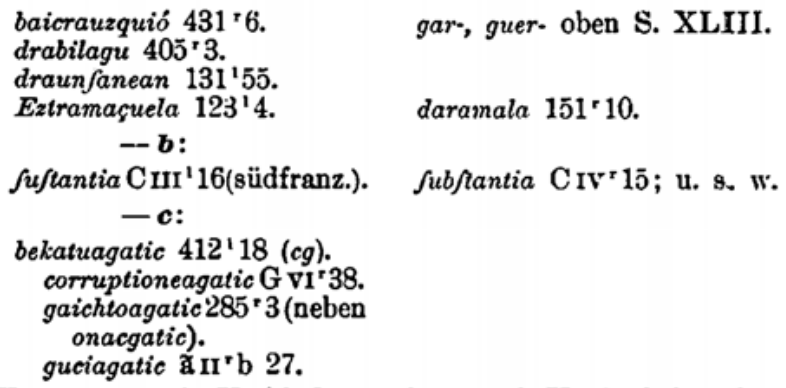

Wo guciagatic, in Verbindung mit -ere, als Konjunkzion (franz. toutesfois) verwendet wird (z. B. 53'39. 305' 11. 351'16. 429'9), lässt es sich ohne Weiteres aus dem Sing. erklären (vgl. span. con todo); immerhin ist zu bemerken dass auch guciacgatic (z. B. **'1'21. A vi'34) so vorkommt.

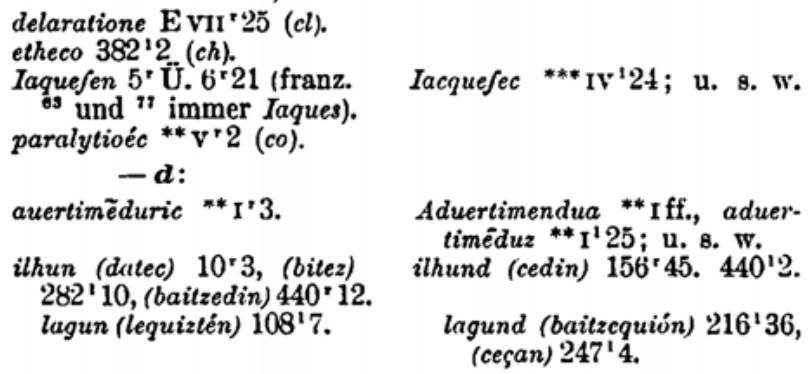
(ceşan) $247^{\prime} 4$.

Der verschiedene Auslaut des Radiksls wird meistens auf der Verschiedenheit des folgenden Anlauts beruhen; doch sollte dann vor $q$ auch nicht $d$ (vgl. noch vrrund /cedin) 152 41 , arind [cesaten] $263^{\prime} 38$ ) geschrieben werden, sondern $t$ (rgl. oben S. XL ehuchent).

- $\boldsymbol{e}$ :

bnedicatua ${ }^{*+} \mathrm{vI}^{1} 38$ (en).

çarri EvII' $29(e q)$.

dclaratu 255 × $22(e c)$.

ftomaquera $190^{1} 25$ (lat.). e eromac $206^{\circ} 20$,-cagatic $37^{\prime 2}{ }^{1} 23$.

Das inlautende präteritale $n$ wird, je nach den Mundarten, bald von dem folgenden Stammlaut durch ein e getrennt, 
bald nicht (s. Bask. Stud. I, 6 f.). Aber innerhalb derselben Mundart ist hiermit eine Funkzionsverschiedenheit verbunden, so bedeutet bei L. z. B. gueneçan: „wir hatten (hätten) ihn“, guençan: „er hatte (hätte) uns" (C ${ }^{1}{ }^{1} 32.33$ : eguin guençançat - recebi gueneçançat.). In Folge des Schwankens zwischen den Mundarten kommen leicht Versehen vor, so: guenģan FI'13. guençaque $\mathrm{FI}^{1} 10$. cinçaqueizte $17 \mathrm{~S}^{\prime} 39$.

guenetzaqueen $\mathrm{CVIII}{ }^{1} 34$.

In allen drei Fällen steht -ç- nicht für -eç-, sondern für -etz(,wir . . . sie" [Pl.], nicht ,wir . . . ihn").

$-g$ :

feynaleren DiIr 27.

feignale $53^{1} 48$; u. s. w.

Im Romanischen begründet. - Die Formen von egon behalten zum Theil ihr $g$, zum Theil verlieren sie es, wobei $o$ zu $u$ zu werden pflegt; dies beruht auf der verschiedenen Lage des Tones $(a g \hat{a}, a ̈)$. So lesen wir bei L. egon, egoiten, dago, dagoca, dagote, dagozca, ago und euqui $\left(165^{\mp} \ddot{\mathrm{U}}^{1} 170^{1} \ddot{\mathrm{U}}\right.$.), daut, dauc, nauçue, dauque, baiquinauteque $\left(\mathrm{A} \mathrm{IV}^{r} 1\right)$, çaudete, aut. Es scheinen aber Schwankungen vorzukommen, von denen ich wenigstens eine aufgezeichnet habe: diaoc $29^{1} 23$.

diagoc 224.11.

$\mathrm{Ob}$ in itzeuquiz ã III'a 22. viI'c 32 . B r ${ }^{\top} 30$ (,für Etwas haltend") $d$ ausgefallen ist oder $g$, wage ich nicht $z u$ entscheiden; dem Sinne nach wäre das Erstere anzunehmen, indessen ist das Vorhandensein eines guip. itzegoqui „angemessenes Sprechen“ nicht zu übersehen.

$-\boldsymbol{h}$ :

amorz $332^{r}$ 18. Kal. $\mathrm{I}^{1} 5$.

aflerre $3^{1} 16$ u. s. w., $a f$ :

Serreiaffunaren $344^{-26}$.

rra G viI' 34 (Wortver-

wechselung, wegen des

folgenden element).

Das $h$ - ist auch im zweiten Falle das Ursprüngliche. Im Allgemeinen wahrt L. $h$-, aber nicht wo es die 2 . P. S. bedeutet (also $a i z, a u$ u. s. w.), mit einziger Ausnahme des Imperativs habil (jedoch im Ind. abila $203^{\prime} 15$, abilala $250^{\prime} 24$ ). Die andern intransitiven Imperative, so adi, ago, athor, oha (auch im Ind. oha 195 5) haben es nicht.

abillamenduac $39^{1} 7$; u. s. w. aborritzen $\mathrm{G}$ III' 17 . hamaborz $185^{\circ}$ '18. 263 r28.

hajJjerretajJiına ${ }^{* *}$ viII $^{r} 18$. 


\section{LIV}

Wiederspiegelung romanischer Schreibweisen. naiz $17^{1} 20$ (Wortverwech-

selung).

Das $-h$ - pflegt bei L. zu bleiben; vgl. bahaiz, bahau u. s. w. $\mathrm{zu} a i z, a u$ u. s. w. In den Formen von ioan erzeugt ähnlich wie in denen von egon (s. vorherg. S.) die Betonungsverschiedenheit verschiedene Behandlung des konsonantischen Inlauts; vgl. oha und doa (badoa), ciohan und cioacen. Auch hier finden Schwankungen statt, so : baguioaçac $205^{\circ} 3$.

guihoarac 117'24. dihoaçac $372^{\prime} 24$; vgl. badihoac $113^{\prime} \mathrm{s}$, nihouc $41^{\mathrm{r}} 30$, banihoac $135^{\circ} 19$.

Weglassung des $h$ nach $p$ und besonders nach $t$ : Blaspemioa $128^{\prime}$ Ü. çorte $155^{1} 34$. garaitu $435^{r} 21$ (in der vor-

herg. Z. garaite). guerta $12^{1} 28$. menta $45^{\text {r }} 23$. Nephtalingo $5^{1} 15$. vrte Kal. I'3. II'1. ${ }^{1} 1, \quad$ vrthe $-15^{1} 20$ u. s. w. Kal. I' 6.7 Vrtén Kal. II ${ }^{\mathrm{r}} 2 .{ }^{1} 2 . \quad$ (zwei M.). II' ${ }^{1} 23.29 .30$.

$\mathrm{Zu}$ garaithu bemerke ich dass wenn es, als Radikal, das $u$ abwirft, auch das $h$ verliert, da th nicht am Wortende stehen kann, also garait $\left(127^{\circ} 22\right.$. $\left.^{2} 85^{\circ} 21\right)$, wie bat $117^{\prime} 27.167^{1} 51$ von bathu.

$$
\begin{aligned}
& -\boldsymbol{i}: \\
& \text { erd (cedin) } 444^{1} \text { ๖े. } \quad \text { erdi (cedin, -no) } 2^{1} 25.100^{\circ} 5 \overline{7} \text {. } \\
& 101 \times 7 \text {. }
\end{aligned}
$$

Allerdings bietet für das sonst übliche erditzen L. - ich bin nicht sicher ob ausnahmslos - ertzen $\left(99^{1} 57.101^{\prime} 6.7 .195^{\prime \prime} 21\right.$. $\left.336^{\mathrm{r}} 19 .{ }^{1} 27.402^{\mathrm{I}} 15\right)$.

ezluquete $194^{\prime} 22$. etzuqueten 293 r8.

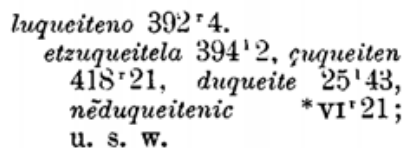

L. scheint hier -quei- vorzuziehen; so auch dacufqueiteno $77^{\prime} 1$. Aber anderseits regelmässig: deçaquete, leçaquete. Vor $z t$ breitet sich -quei- noch weiter aus:

$$
\begin{aligned}
& \text { etzintuquezten } 21^{\circ} 7 . \\
& \text { ģaituqueztenean } 7^{\circ} 11 .
\end{aligned}
$$


caitzaquezte $174^{17}$, saitzaque ztenari $290^{1} 25$.
cinçaqueizte $17 \mathrm{~S}^{1} 39$, baititza. queizte 67 '32, baitzitza- queizten $62^{1} 2.395^{\circ} 11$, litza. queizten $213^{\prime} 21$.

So findet sich ei- auch ausserhalb des Potenzials, z. B. cindeiztençát oben S. XLIX. Endlich liebt es $i$ nach denı Stammauslaut $o$ vor te aufzutreten:

diroiteno $32 \cdot 28$, ciroitenaren $67^{\prime} 33$, liroiten 109 ' 19.

cioten (-ela) $14^{\mathrm{r} \cdot 25}$ u. s. w. cioiten (-ela) $14 \cdot 29$ u. s. w.

(wie es scheint nur im

Anfang).

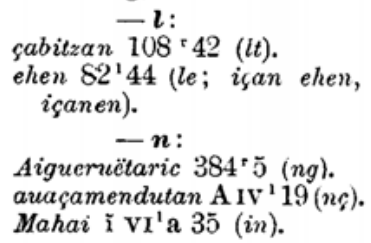

Es hat wohl hier überall Verwechselung zwischen dem einfachen Vokalzeichen und dem mit Tilde versehenen stattgefunden.

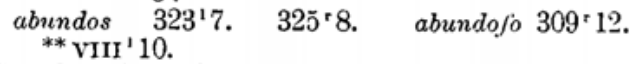

Gewöhnlich erscheint diese Adjektivendung in spanischer, nicht in südfranzösischer Form: auaritiofo, curiojo, guthiciojo, inuidiofo, mifericordiojó u. s. w.; doch abundojqui, deliciofqui perilofic $377^{\mathrm{r}} \mathrm{1}$.

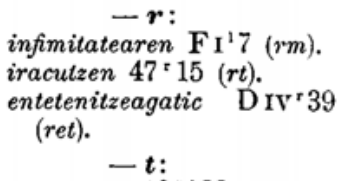

borhitzago $126^{1} 22(t h)$.

ohoitzá Abc B II ${ }^{1} 24$ (th).

Die Nachbarschaft eines zweiten $t$ hat den Ausfall des $t$ offenbar begünstigt. Alle die Fälle in denen $t$ vor einem $z, f$ oder $c h$ fehlt, müssen besonders und mit denjenigen in denen es unter denselben Umständen überflüssig ist, zusammengestellt werden; denn das Lautliche spielt hier die Hauptrolle, und 


\section{LVI}

weder das Ursprüngliche noch das Regelmässige ist überall mit Sicherheit zu bestimmen.

э) zwischen Vokalen:

cerbizatzen 350 r 22 (cerbitza. tzen in der vorherg. Z.). ** Ir22. E $\mathrm{II}^{\prime} 20$ (hier $z a$ am Zeilenanfang).

ezticaçuela 364 r20 (tza; es steht unmittelbar darüber ezteçaçuela).

gaizetfi ${ }^{*}$ VIII 120.

hizetic $280^{1}$ Ü.

menofpreciaçale $377 \times 2$. recebiçale $380^{\circ} 8.413^{\prime} 9$

(-rale ist dlas Gewöhn-

liche: difpenfugcale, tra-

dicale u. s. w.).

nazayó 333 ' 19.

gaitzet/fi ${ }^{* *} \mathrm{VI}^{1} 10$.

menofpreçatzalecic recebitzaleac 39 г $\mathrm{U}$.

natzayo $384^{15}$ u. s. w. vrthetacozat $129^{1} 19$. vziten D III ${ }^{1} 34$.

Auch hier geht meistens ein anderes $t$ vorher oder folgt. Auslautendes $t z$ wird im Inlaut oft zu $c$, 80 gatz "Salz": gaciten "salzen“; in Zusammensetzungen wird hier das Ursprüngliche gewahrt, dort nicht - gaicetsi verzeichnet van Eys als lab., gaitzetsi als niedernav. Auch sonst verhalten sich die Mundarten verschieden; so entspricht dem natzayo L.s anderswo nicayo (nitzayo). Bei vziten könnte die mdl. Variante uzten mitgespielt haben; bei cerbizatzen die Verben mit griechischer Endung, wie euangelizatzen, fcandalizatzen. Was $t f=f$ anlangt, so habe ich dafür hier keine Belege; für $-t / u$ habe ich ein paar Mal $-s u$ gefunden (s. oben S. XXXVI), $-f u$ nicht. Hingegen wechseln zuweilen $t c h$ und $c h$ miteinander, wobei nicht übersehen werden darf dass die spanische Schreibung ch und die französische tch gleichwerthig sind. So:

errachago $37^{2} 24$; u. s. w.

probechatzera ** VIII ${ }^{1} 34$.

reprochuric $97^{\prime} 6$.

uncichobat $64^{\prime} \mathrm{g}$. erratchago $15^{\circ} 5.63^{\circ} 9$, -tchenic EvII ${ }^{1} 13$.

probetchatzen $56^{12} 24$; u. s. w. reprotchu $360^{1} 10$; u. s. w. uncitchoric $171^{1} 22$ (vgl. oben S. XL).

Es gibt im Baskischen zwei Verben welche sich sehr ähnlich sehen und von denen das eine „halten“, das andere „brennen“ bedeutet. Bei L. lautet in der Partizipform jenes etchequi (etchequidurá $\left.75^{r} 35\right)$, dieses iechequi ( $\left.\widetilde{\mathrm{e}} \mathrm{III}^{\mathrm{r}} \mathrm{b} 19\right)$, die Faktitive 
dazu : eratchequi ,hinzufügen", irachequi ,anzünden". Beide Verben sind aber aus einem differenziert; eine mundartliche Lautverschiedenheit hat sich, wie das auch sonst im Baskischen vorzommt (vgl. o. S. XXXVII ekarri, egari), mit einer Bedeutungsverschiedenheit rerknüpft. Das ergibt sich insbesondere aus der Konstrukzion. Unserem transitiven Verb entspricht im Baskischen ein intransitiv-zielendes; statt "haltet ihn" heisst es ,haftet (an) ihm", "heftet euch an ihn" (so 53'48. 92'44); on denari satchetzate "behaltet das Gute" (364'21) ist eigentlich so viel wie "haltet fest am Guten". So wurde denn auch gesagt: „das Feuer haftet oder heftet sich an Etwas" (vgl. span. port. pegar), und dafür kurzweg: „es [unp.] haftet an Etwas“" $=$ "Etwas brennt" (8. Bask. Stud. I, 41 f.). Deutlich scheiden sich nun die einen Fälle von den andern; eztatchecularic buruäri $356^{\prime} 19$ ist : ,ne retenant point le chef" (,,hält sich nicht an dem Haupt " übersetzt Luther), und candela sachecana $170^{r} 35$,die Kerze welche brannte". Einmal jedoch scheint L. das Verb mit -ch- im Sinne des mit -tch. gebraucht zu haben: guciey baitacheté Iaincouren zeloa $250^{1} 20^{\circ}$,Alle hält (hat erfasst) der Eifer des Herrn" (dieselbe Form: Ene manamenduey datchetena $192^{12} 21$ „Der meine Gebote hält"); im Griechischen, Lateinischen und Französischen ist das ganz anders ausgedrückt, also keine Auskunft zu holen. Es könnte ja auch gemeint sein: "Alle entzündet der E. d. H."; würde das aber nicht eher so gesagt worden sein: guciac irachequiten baititu Iaincoaren zeloac?

B) nach einem Konsonanten. Bekannt ist wie nach $l$ und $n$ vor einem (stimmlosen) dentalen Reibelaut $t$ ebenso leicht unterdrückt als erzeugt wird und dass die Schreibung das oft in umgekehrter Weise ausdrückt. Die Infinitirendung lautet bei L. nach $l$ (ebenso wie nach $r$ ) tzen (biltzen, hiltzen, hurbiltzen), nach $n$-cen (hancen, kencen, laguncen), während man heutzutage auch im letzteren Falle -tzen zu schreiben pflegt. Die Endung -sale, -tzale (vgl. oben S. LVI) scheint sich nach den Infinitiven $\mathrm{zu}$ richten (z. B. hiltzaleacgatic $368^{\prime} 9$ ), aber wir finden doch:

salçaleac $162^{\prime} 14$, -ley $163^{\prime} 16$. Saltzaleac $39^{\prime}$ Ü. 162' Ü., saltza.

Man bemerke ferner noch:

alchatu $100^{1} 69$, -tzen $365^{\prime} 4$. altchatu $20^{\text {r. } 23}$; u. s. w.

${ }^{* *} \mathrm{IV}^{\mathrm{r}} 31$. EV $\mathrm{V}^{\mathrm{r} 2}$, -tzera

* VIII* 28.

guelsurrunac 433'23. Gueltzurrunac ì II $^{1} \mathrm{~b} 5$.

Augenscheinlich liegt hier eine Zusammensetzung vor: gueltz-, guiltz-, gultz-, guntz-, kuntz-, wie die verschiedenen Mundarten 


\section{LVIII}

bieten, + urrunac, was wohl nichts Anderes ist als das sonstige errainac, errañac vom lat. renes. Nach $n$ ist $\xi$, ch (aitzinchetic $243^{\text {r2 }}$, ebenso wie harache $53^{\times} 36$ u. ä.), $f$ (hurrenfu $261=28.29$ - Pouvreau schreibt hurrentfu -, iaquinfu $416^{\circ} 12$, vkanfuago $300^{\prime} 8$, während nach Vokal $-t / u$ steht) das Regelmässige. So auch guens-, cins- für guenetz-, cinetz- oben S. LIII. Doch umgekehrt: cintzatêçát $354^{1} 22$. erauntfiz $171^{1} 18$. cinçatençat 212 r 26 .

eraunfi $12^{1} 25$. 27 , draunfanean $131^{\prime} 55$.

Nach $r$ fällt Setzung oder Nichtsetzung von $t$ schwerer ins Gewicht: iracurçaléc ${ }^{* *} \mathrm{IV}^{1} 29 . \quad$ iracurtzen $88^{1} 14$; u. s. w.

Im Romanischen wurzelt die Schwankung zwischen: Martchantac $\tilde{\mathrm{i}} \mathrm{VII}^{\mathrm{*}} \mathrm{a} 1$, an- marchãt $26^{\mathrm{r}} 45$, -antaren ì $\mathrm{II}^{\mathrm{l}} \mathrm{b}$ dica $\tilde{\mathrm{i}} \mathrm{VI}^{1} \mathrm{~b} 46$. 31 , -andica $452^{1} 12$.

$\gamma)$ vor einem Konsonanten der Ableitung pflegen $t z$, $(t c h$,$) tf ihr t \mathrm{zu}$ verlieren, z. B. eritzi erizle, gaitz gaizqui, abrats abraftaffun, ihardetfi ihardeften. Ein paar Verstösse dagegen: anhitztajfuna $413^{\prime} 8$. çorrotzqui B III ${ }^{1} 28$. gaitzet/le $284^{1} 9.377^{\circ} 3$. ebatfleacgatic $368^{\mathrm{r}} 10$. gorrozqui $\mathrm{B}$ vir ${ }^{1} 20$. onhetfle $380^{\circ} 8.412^{\circ} 8$. onhetstẽ $380^{1} 4$. othoitztebat $\tilde{\mathrm{a}} \mathrm{V}^{\mathrm{r}}$ a 2 . gaitzej'le $267^{\prime} 30$.

onhejtea $86^{1} 33$; u. s. w. othoiztez 69 r 18 ; u. s. w.

Vielleicht eine Vermischung mit othoitzbat. Deun mit diesem Verb verhält es sich eigenthümlich. Das ursprüngliche Radikal dazu ist othoi; wir finden es in othoi citzaten $234^{\circ} 42$, und sodann als Einschaltungswort, wie unser "bitte", so A I ${ }^{1} 9$. II ${ }^{r} 18$. $35 \mathrm{u}$. s. w. (daraus ist das Fragewort othe entstanden). Das Partizip und der Infinitiv dazu: othoitu, othoitzen sind mir bei L. nicht aufgestossen (das Soulische besitzt sie), wohl aber das diesem Infinitiv zugrunde liegende Verbalsubstantiv othoitze, so othoitze $249^{r} 36$, -eac $369^{r} 1$, und in Verbindung mit eguiten: $152^{\circ} 32.217^{\circ} 6.231^{\circ} 12.249^{1} 5.347^{\prime} 4$. Meistens aber wird othoitze in diesem Falle zu othoitz verkürzt (und nicht etwa bloss vor vokalisch anlautenden Formen, z. B. othoitz daididano $53^{\circ} 36.91^{\circ} 32$ ), während es sonst gern durch othoitza vertreten wird: othoitzá $97^{1} 13.239^{1} 13$, othoitzaz $352^{1} 6$, othoitzara $239^{1} 16$. Othoitza $249^{\prime}$ Ü., othoitzac $388^{\prime} 7$ (für -ác?) sind zweideutig; othoitzetara $412^{\prime} 12$ sogar mehrdeutig. Im Anschluss an dieses othoitz eguiten (in rascher Aussprache othoitziten) wandelte sich das ursprüngliche othoitzen in othoizten um (Umstellung von zt zu $t z$ ist nicht ganz selten; vgl. bei L. ikvtzen zu ikuci, 
heute ikuzten), und dazu stellte sich nun regelrecht als Radikal othoitz, so othoitz cegan $108^{\prime} 12$, othoitz citzaten $240^{\prime} 39$. Anderseits wurde othoizte ganz so wie othoitze, othoitz mit eguiten verbunden: $208^{\mathrm{r}} 24.227^{1} 30.232^{\circ} 3$.

$\delta)$ am Wortschluss :

borthiz 126 ' 21 .

dohain gaiz 149'23. borthitz 22 × 29 ; u. s. w.

dohain-gaitz 47'19, dohaingaitz $88^{1} 17$.

In allen drei Fällen folgt emazte. Umgekehrt:

hunelacotz $\mathrm{ArV}^{1} 6$.

Verwechselung mit der so häufigen Endung -cotz.

Endlich ist $t$ am unmittelbaren Wortende weggefallen:

duçuença $170^{\circ} 40$.

Das ist zwar ein wirklicher Druckfehler; doch es muss dabei auf einige Gruppen mundartlicher Varianten hingewiesen werden. Das Suffix $-r a t$, welches sich aus $-r u+t$ zusammensetzt, hat sich mit dem Suffix -co zu -cotzat verbunden. Neben diesem besteht nun -cotz (für *-co-tza), welches mit Unrecht als eine mechanische Verkürzung von -cotzat betrachtet wird, denn beide haben ursprünglich, wie zum Theil noch heute, verschiedene Bedeutungen. Bei $\mathrm{L}$. sind sie gleichwerthig; doch ist -cotzat bei Weitem das Seltenere, vielleicht das Alterthümlichere; ich gebe ein paar Beispiele davon: bidecotzat, át $17^{\circ} 10.71^{\circ} 8.119^{\prime} 3$, egunecotzat $291^{\prime} 8$, eternaleracotzat $368^{\prime} 16$, hartacotzat $108^{\mathrm{r}} 43$. $\mathrm{a}^{\mathrm{r}}$ a 12 , ohorztecotzát $51^{1} 12$. Ganz entsprechend ist das Verhältniss von -rat und -rat (-gana und -ganat). $\mathrm{Ob}$ das folgende Wort mit einem Vokal oder Konsonanten und mit welchem anlautet, hat dabei keine Bedeutung; wir finden sogar nora-ere $73^{\prime} 56.90^{\prime} 14$ neben norat-ere $13^{\prime} 19.12^{\prime}{ }^{\prime} 57$. $33^{1}$ 6. $447^{\circ} 4$. Immerhin mag hier die Form mit $-t$ die häufigere sein, wie sie das in manchen andern Fällen ist, z. B. guibelerat, cerurat. Aber bei den meisten Wörtern überwiegt ersichtlich die Form ohne $-t$ die mit $-t$; ausschliesslich herrschend ist sie vielleicht nur bei den Infinitiven (emaitera), selbst die fremden Ortsnamen verschmähen das $-t$ keineswegs, und so haben wir z. B. Cyprerát, Damajcerat, Phenicerát neben Cyprera, Damajcera, Phenicera, so ein paar Mal Ierufalemerat neben häufigstem Ierufalemera. Bei Antritt von -co wird -rat- bevorzugt, z. B. baitharatco $248^{1} 21$, batetaratco $\mathrm{CrV}^{1} 32$, Ierufalemeratco $133^{\mathrm{r}} 22$; selten fehlt $-t$, z. B. baitharaco $350^{\prime} 30$. Ein gleiches ist das Verhältniss zwischen -ganatco und -ganaco; letzteres z. B. in gureganaco $\mathrm{B}$ Vin' ${ }^{1} 22$. F $\mathrm{I}^{ } 16$.

$-z$ :

eliçate EvII ${ }^{*} 1.2$ (zl; soul.). 
Ich stelle die Fälle besonders in denen, offenbar während des Druckes, der erste Buchstabe eines Wortes und zugleich der Zeile geschwunden ist:

$u c$, oun $26^{1} 57$ R. (l-, $i$-).

ongit $73^{\circ} 45(c-$; der Platz

dafür ist $d a)$.

uc $199^{1} 33$ R. (l-).

guna ã vi $\mathrm{r} 14(e-)$.

Weil in derselben Weise entstanden, sei hier der Wegfall der Zahlen (26. 7 - 12. 3) erwähnt in:

Mat )

berr. $184^{\mathrm{r}} 2 \mathrm{R}$.

b) von Vokalen neben Vokalen. Hier lässt sich zum grossen Theil an gesprochenen Vokalschwund, bez. Vokalzusammenziehung denken.

- $a$ :

bacequiagu $85^{1} 14.181^{\prime} 24$. baguitza $423^{1} 20$ (mdl.; oder $u$ für $a$ ?).

ezquitzaizte $332^{1} 5$ (mdl.). baititezque $441^{1} 20$ (mdl.); vgl. baditez $324^{\prime} 4$. cerbitzun $151^{1} 27$ (ua). Chanango $\mathrm{D} \mathrm{vi}^{1} 13$. extrordinario $\mathrm{G} \mathrm{VI}^{\mathrm{i}} 25$. guça $323^{\circ} 16$ (au; es folgt unmittelbar gucietan).

hourrey $\mathrm{DIV}^{\mathrm{r}} \mathrm{13}$ (aon). ioiten 8' 32 . 12' 53 (oai; Verwechselung mit ioiten "schlagen").

Ionnefgana $\mathrm{i}^{\mathrm{T}} \mathrm{a} 2 . \mathrm{\sigma}^{\mathrm{I}} \mathrm{I}^{1} \mathrm{~b} 32$ (oa).

nuqueano 191'38 (au; wohl mdl.).

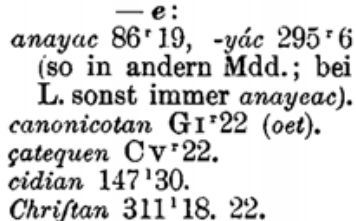

baceaquiagu $42^{1} 16$ u. s. w. ezgaitzan 324 ri20.

gaitzaizte $295^{\prime} 9$. daitezque $148^{\circ} 36$, -quec $372^{1} 25$.

Chanaango $232^{1} 19$.

extraurdinarioqui $\overline{\mathrm{a}} \mathrm{vII}^{5} \mathrm{~b} 6$. çatequeen $262^{\prime} 21$.

ciedián $147^{1} 32$. 
Offenbar für das sonstige Chriftean, also die Form mit dem Artikel = franz. en Chrift, während ausserdem auch le Chrift vorkommt; für Chriftan, nach Analogie von Adamtan ebenda, Moyfeftan $302^{\prime} \cdot 2$, wird es nicht stehen; ohne Artikel sagt L. Chrift baithan.

ditzaquela $\mathrm{G} \mathrm{vI}^{\mathrm{r}} 40$ (ee; $\mathrm{s}$. unten S. CIX).

Ifralen $13^{*} 10(a e)$.

$-\boldsymbol{i}$ :

compania- $114^{\mathrm{r}} 9$. ã $1 \mathrm{II}^{\mathrm{r}} \mathrm{b} 28 . \quad$ compainia. $109^{1} 29.400^{\mathrm{r}} 22$. $41 \bar{r}^{\mathrm{r} 9}$. a $_{\mathrm{II}}{ }^{\prime} \mathrm{b} 33 . \mathrm{v}^{\prime} \mathrm{a} 29$. VI'b 21 .

dethua $299 \times 22$ (ei).

ejcunean $92^{1} 62$ (ui).

ezpetarreicu $79^{1} 38$ (ezpei-

tarreicu geht kurz vorher). iarrequi $64^{1} 7$ (ei).

menofprę̧atzaleác $234^{\circ} 41$.

menofpreciaçale $377^{\mathrm{r}} 2 ;$ menofpreciatzen regelm.

$-\boldsymbol{u}:$

difcipuléc 184 ' 12 (ué).

dracun $423^{\prime} 23(a u)$.

Ist doch vielleicht nicht, wie van Eys VAL S. 40 Anm. annimmt, ein wirklicher Druckfehler, sondern eine mundartliche Form; Axular ersetzt in diesen Formen au durch $a$, wenn die folgende Silbe $u$ hat, also deraut, derauca, derauzquigute, aber deracu, deratçu, baleratsu, und ähnlich haben die westniedernav. Pregariac von 1651 (BB 23d) darauciet, darauzquicien, aber darucogu, daratçogun (und sowohl daragute als baitaraucu). liburän $334^{\mathrm{r}} 10(u \ddot{a})$.

paffutara FviII ${ }^{1} 15$ (auf;

Wortverwechselung).

veztimendetaco $44^{15}$ (uet).

nié (eznié) $218^{\mathrm{r}} 7.256^{1} 12$. niaué $378^{1} 16$; rgl. niauc $257 \times 18$ (soul.). $224^{1} 17.375^{\prime} 17$.

c) Vereinfachung von Doppelkonsonanten: $\boldsymbol{l}$ für $\boldsymbol{\imath l}$ :

muthilén $209^{\circ} 18$.

Solennelagatic a $\mathrm{VII}^{\mathrm{r}} \mathrm{a} 5$.

muthillén $48^{\prime} 45$; u. s. w.

$\boldsymbol{r} \boldsymbol{r}$ für $\boldsymbol{r}$ :

beldurequin $\mathrm{D}^{1}{ }^{1} 6$.

bigáren $393^{\prime} 13$. 


\section{LXII}

egari $212^{r} \ddot{\mathrm{U}}$.

egari s. S. XXXVII.

eraitera ${ }^{* *}$ VIII ${ }^{*} 30$.

ethori ẽ VII ${ }^{\mathrm{b}} \mathrm{b} 41$.

gorharats $444^{\circ} 3$.

haourér ã VII'a 44.

gorrhatsbat $437^{\circ} 4$.

Iareiquiteco ĩ $\mathrm{IV}^{\mathrm{r}}$ a 40.

interoyatzè $146^{\circ} \mathrm{U}$.

muraillác $457 \times 14$.

murraill- $224 ' 25 . \quad 45612$.

$457 \times 15.17 .18 .19$.

Das $r r$ ist hier echtbaskisch ebenso wie in murru- $328^{\circ} 33.398^{\prime} 30$.

d) Schwund ganzer Silben:

$$
\text { - } \boldsymbol{a b} \text { : }
$$

amorz $332^{r} 18$. Kal. $I^{1} 5$ hamaborz $185^{\circ} 18.263^{\circ} 28$.

(vgl. hamortz Rev. de ling.

III, 432).

$$
\text { -ct: }
$$

circonfioncaren $\quad 272^{\circ} 12$. circoncifionecoén $272{ }^{\circ} 12$. $287^{\circ} 8$.

vitzen $275^{19}$ (ici).

$$
\text { -de: }
$$

batberac B III ${ }^{1} 27$ (ede).

$$
\text { -ga: }
$$

hobeagatic ${ }^{* * *} \mathrm{VII}^{\mathrm{r}} 1$.

hobeagoagatic A II'34. B VII ${ }^{1} 5$. - ha:

çarra ẽ vi'b 17.

çahara $455^{\circ} 2$.

Hierzu ist zu bemerken dass çar allerdings eine mundartliche Form von gahar ist, doch, wie das auch sonst im Baskischen vorkommt, neben ihr in etwas anderer Bedeutung gebraucht wird. Salaberry gibt für das Niedernavarrische şhar "alt" i. e. s. an, sar, alt" im verächtlichen Sinne (,très-commun, de peu de valeur, vieux, use" "); van Eys hält diese Unterscheidung für willkürlich, aber sie findet sich bei L. bestätigt - man

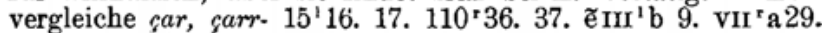
ivi a 43 und çahar, şaharr- $26^{\times} 52.110^{r} 39.274^{\circ} 6$ u. s. w. An den beiden oben angeführten Stellen ist die kürzere Form im gleichen Sinne gesetzt wie die längere, oder doch auf das Gleiche bezogen; denn "die alte Schlange" verträgt eine mehrfache Auffassung.

- ta:

chariteari $308^{1} 1$ (Einmisch- charitatea $47^{r} 12$; u. s. w. ung von franz. charité).

libertatera FVII' 15 (teta).

Wird nicht die best. Dekl. sein, da cerbait pajutura vorhergeht. 
3) Zutritt von Zeichen:

$$
+a:
$$

aincen 22: $4^{\prime} 17$ ( + entspr.

Praes. aicen).

auen $214^{1} 4$ (+ Praes. auen ,dich ....".).

bethau $214^{1} 4$ (+ Pracs. bahau ,dich .....").

bainoa $190^{*} 16\left(a_{2} ;+\right.$ bainoa von ioan?).

egunean $257^{\prime} 24$ (unmittelbar vor buruän).

işanean 212 ri23 (ea; +icenean?).

gauciaren $242^{\prime} 26\left(a_{1} ;+\right.$ gau. garen; vgl. guça S. IXX). nahaiz $109 \cdot 18\left(a_{2}\right.$; vgl. naiz S. LIV).

Es ist möglich dass daccurraçuen 193' 8 eine mundartliche Form für *dacarçuen ist; ich weiss $z$ war nicht ob dies bei $\mathrm{L}$. vorkommt, aber er sagt wenigstens ekarçue $78^{1} 19.205^{5} 10$ neben ekarrac $122^{r} 41.378^{1} 13$, wie ecarçue $149^{\circ} 14$ neben eçarrac $198^{\prime} 11$. $204^{1} 27.448^{\circ} 18$. Im Allgemeinen begünstigt er allerdings den „Bindevokal"; er hat nabila, dacuffat für das nabil, dacust anderer Mundarten.

$$
+c \text { : }
$$

gathibatuac G II '26 (unmit-

telbar vor non I(iincoac).

guciacgatic $\mathrm{Abc} \quad \mathrm{B} \mathrm{v}^{\circ} 26$

$\left(c_{2} ;\right.$ nach eure populu;

vgl. -agatic für -acgatic

S. LII).

hitzaric $249 \cdot 32$ (unmittel-

bar vor cein).

Iehofuach ** VII' 37.

prefumitzceco $\mathrm{Av}^{\mathrm{r}} 20\left(c_{1}\right)$.

Kein überflüssiges $c$ steckt, nach L.schem Sprachgebrauch, in guigonec batec-ere $373^{1} 16$, beroc batec-ere $439^{1} 16$, wenn auch $\mathrm{TB}^{28}$ es in guiçon batec ere, bero batec ere umgeändert hat.

$$
+d \text { : }
$$

cindeçaquete $177^{1} 19$ (niedernav.; es geht vorher baninduçue).

cineşaquete $326^{1} 4$. baitzineçaqueten $293^{\prime} 2$. 


\section{LXIV}

$$
+e:
$$

baceaquian $56^{\mathrm{r}} 18.94^{\circ} 10$, baçaquĩa $163^{\mathrm{r}} 25$, laquiançat baceaquitela $108^{\circ} 41$, ba- $144^{1} 15$; u. s. w. ceaquizquian 111 \% (mdl.). çayene ** I'24.

Es folgt unmittelbar remõftrançaz; sollte das dem $r$ vortönende $e$ hier zum Ausdruck gekommen sein? cembaterebeit $229^{\prime} 13$. cembatrebeit $239^{\prime} 12$.

Zwar ist bat-ere das Ursprüngliche, aber batre ist die durchweg herrschende Form. eduqueiten $\mathrm{C}_{\mathrm{III}}{ }^{*} 34$ (wohl eduquiten $73^{1} 3$ u. s. w. $\sim$ erideiten u. s. w.).

Ieoean Kal. VI' 16 (vgl. Concilioan ebend. 11, Virgoan ebend.' 14).

sichele $444^{17}$.

reguelá $35 \overline{5} 5^{1} \overline{\text { J. }}$.

$+\boldsymbol{h}$ :

Michel $429^{\circ} 9$.

regla $338^{\prime} 16.351^{\prime} 16$.

arthatjean s. oben S.XLI.

azphian $\tilde{\mathrm{e}} \mathrm{III}{ }^{\mathrm{r}} \mathrm{a} 21$.

baithitu $342^{\circ} 9$ (bethidanic, baithan vorher in demselben Vers).

gaithu $299^{\text {× } 15}$ (unmittelbar nach deithu). gaithuán Abc $\mathrm{B} \mathrm{IV}^{1} 17$

(unmittelbar vor othoi). gau-herditã $49^{\circ} 6$, gauherditan $89^{\prime} 35$, gauherdi $247^{1} 7$.

onherizte $\mathrm{D}_{\mathrm{VIII}}{ }^{1} 4$. ĩ $\mathrm{VIII}^{1} 6$. On eritzi ĩ vIII'12; u. s. w. 30 ; u. s. w. onherranen $112^{\mathrm{r}} 26$.

Es könnte sich bei gauherdi um ein ,hiatustilgendes“ $h$ handeln; aber wie ist es nach on zu erklären (wir haben auch onhetfi, onhaffun)?

Phamphyliara $236^{\circ} \cdot 24\left(h_{1}\right)$.

Vgl. noch oben S. XLI hartoca für arroca. $+\boldsymbol{i}:$

aitzaitadan $71^{1} 23$.

alegranciázco $323 \times 2$. puiffanciaco $365^{\circ} 7$.

Latinisierend; vgl. S. XXXII.

çaitzaizqueten $430^{\circ} 24$. saitzaquezte $174^{17}$.

\section{atzait $31^{1} 23$.}

alegrançan $49^{\prime} 21$; u. s. w. puijłança $340^{\text {r21 }}$; u. s. w. 


\section{LXV}

S. oben S. LV und unten S. LXVIII. cembaitrabeit $257^{\prime} 24$. cembatrebeit $239^{\prime} 12$.

daichecanera $25^{\prime} 42.26^{\circ} 50$, dachecan $456^{\prime} 8$, irachequiten iraichequiten $7{ }^{\circ} 15$. $405^{\mathrm{r}} 5.6$; u. s. w.

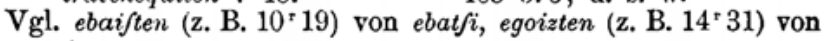
egotzi.

dieçaqueo $10^{1} 27$ (mdl.).

gaiztigamendua $433^{1} \mathrm{U} .\left(i_{1}\right)$.

deçaqueodano $132^{\prime} 8$, deçaqueogu $361^{1} 9$, gueneçaqueoguiciuc $270^{\circ} 9\left(i, i_{1}\right)$.

Hilitzecíz $6^{1} \ddot{U}^{\prime}\left(i_{2}\right)$.

Hilitzera i $\mathrm{III}^{1} \mathrm{a} 36$.

ihardieften $80^{1} 5$ (+ ar. ihardeften $80^{1} 4$ u. s. w. diejten).

iairreiquiten $83^{\circ} 52\left(i_{2}\right)$.

maintenitzen $437^{1} 9$,

D III' $^{\prime} 10$ (franz.).

ohortziten GIV'13.

On eritziteco ĩ viII 'b 15.22 ,

-coago ebd. 25 .
Umgekehrt gebraucht $\quad$ L. regelmässig viziten, das wenigstens naren $250^{\circ} 14$; u. s. w. heute weiter verbreitete uzten habe ich bei ihm nicht gefunden. Saincluariotic $449^{1} 7$ (franz.). Sanctuarioco $392^{\circ} 2$; u. 8. w. $+\boldsymbol{l}:$

chilkiratu $36^{\prime} 12$.

ilkuffiren $288^{\prime} 21$.

chikiratu, -uac ebend. (vier Mal).

lieģal-|gunçat A VIII ${ }^{1} 37\left(l_{2}\right)$.

$$
+\boldsymbol{n} \text { : }
$$

guẽquinztén $239^{\prime} 13(n)$.

Wie van Eys VAL S. 60 Anm. 3 dies als ,flexion familière feminine $e^{i t}$ bezeichnen kann, verstehe ich nicht. - Wenn er ebend. S. 31 guinande und guinade gleich setzt, so ist das ein Irrthum; das erstere ist Nebenform von guinate Kond., hingegen guinaden von guinen Impf., baguinade von baguina Supp. Impf.

inharroffiric $234^{1} 51$ (mdl.). iharros $17^{1} 14.71^{\mp} 11.120^{\circ} \overline{5}$.

Ian $\mid$ incoare $\tilde{\mathfrak{a}} \mathrm{v}^{1} \mathrm{a} 7\left(n_{1}\right)$. $240^{\circ} 26$.

$+\boldsymbol{p}$ :

compuntquide $374^{\mathrm{r}} 18$.

Dass es comuntquide heissen muss, ergibt das lat. communicare, franz. communicatifs dieser Stelle; es hat sich compondu ein- 


\section{LXVI}

gemischt, das in der Bedeutung mit comundatu zusammentrifft (vgl. componde, compondura $=$ comunde, comundança ,composición, acomodamiento").

$+\boldsymbol{r}$ :

hayr 303'11 (Einmischung

von soul. hayer).

Spriritu B vI ${ }^{1} 10\left(r_{1}\right)$.

finhefls ${ }_{* * *}^{+S^{\prime} \mathrm{II}^{1} 18.25 \text { (Ein- }}$

mischung von finheften).

vnguenftutzen $115^{1} 38$.

Sonst pflegt in diesem Sinne unctatzen zu stehen. Natürlich liegt lat. unguentare zu Grunde, doch schwebte ein * unguentuztatzen vom Subst. unguentu vor, nach Analogie von damuztutzen, ahalqueztutzen, gueçurzlatzen, odolztatzen.

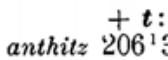

$+u:$
actu. $\tilde{\mathrm{ov}} \mathrm{a} 40$ (unmittelbar

nach refufcitatu).

$+\boldsymbol{y}:$

ezliqucye 171:7 (niedernav. eztiquee $443^{\circ} 9$; vgl. citzaqueé soul.). $256^{\prime} 13$.

peituya ${ }^{* *} \mathrm{I}^{\mathrm{r}} 24$ (niedernav.). peituan ${ }^{* *} \mathrm{vin}^{1} 11$; u. s. w. $+z$ :

etzcheco, $89^{\text {r }} 35$ (unmittelbar nach noiz).

ezgaiztecela $\mathrm{B}$ IV' $28 \quad\left(z_{2}\right.$; wegen des $z$ der vorhergehenden Silbe).

b) Verdoppelung von Konsonanten: $\boldsymbol{b b}$ für $b$ :

BBaina $\mathrm{Dv}^{1} 7$.

$\boldsymbol{l}$ für $\boldsymbol{l}$ :

Afflligituey a viII' $\mathrm{b} 22$.

Gentillén $411^{\circ} 12$.

Gentiléc $17^{1} 18$; u. 8. w.

hillebetheac Kal. II'30. hilebetheã Kal. I'3; u. s. w.

Bei L. scheint die Mouillierung des $l$ nach $i$ nicht durchgeführt zu sein; er schreibt regelmässig muthilla, aber| dabila u. s. w.

$\boldsymbol{p p}$ für $\boldsymbol{p}$

chippiéc $446^{\prime} 16$.

luppecoén $349^{1} 10$.

lupeã $\mathrm{D}$ III ${ }^{\ulcorner} 7$. 
$\boldsymbol{r} \boldsymbol{r}$ für $\boldsymbol{r}$ :

bateturra $71^{\circ} 10$.

error $303^{r} 12$, erroriren $\tilde{\sigma}$

$\mathrm{I}^{\mathrm{r}} \mathrm{b} 43$.

eurrorrec $112^{1} 42\left(r r_{1}\right)$.

itfajforraco $5^{1} 15$.

lekorrerat $49^{1} 15$.

Gesprochen wurde das $r$ in fierretaco ${ }^{* * *} \mathrm{II}{ }^{\prime} 11$, von franz. fier, fière; auch heute noch fierra da, il est fier".

c) Hinzufügung von Silben: $+a c:$

badietzacogu D vi' 11 (mdl., sonst auch -akiogu, -ayogu).

$$
+c a:
$$

Apocacalypsea *** IV'31. $+e n:$

hurrenègo '234'44. 248'15

(= hurrengo + hurreneco, dies $234^{\prime} 42$ ).

(vicitze) $+\boldsymbol{r a}$ :

$167^{*} 36=(v$.) eternale. cotzat, z. B. $166^{\mathrm{r}} 14+$ (v.)

eternalera, z. B. $51+46$.

gorharats $444^{\prime} 3=$ gorrhats 437 ' 4.

Ich halte jenes für eine umgekehrte Schreibung (nach ara, gespr. $a a, \bar{a}$ ); wie pherdats $437^{\prime} 8 \mathrm{zu}$ pherde, so steht gorrhats (Fabre hat: gorrast) zu gorri. Indessen ist eine wirkliche Formerweiterung nicht undenkbar; vgl. marrumalari $414^{1} 8$ neben sonst üblichem marrumari (zu marrumatzen; bei L. marrumaz EviI $\left.{ }^{1} 5\right)$. $+\boldsymbol{r i}$ :

dauritzan F VIr'18.

Dies steht für dautzan $=$ dagotzan (dagoca „er bezieht sich darauf"); ganz ebenso haben wir bei Dechepare AII'18 darauritzut für darautzut, ich habe sie Euch". Das $i$ stammt aus dem Pluralzeichen -it- \}-ti- (8. Bask. Stud. I, 32 f.); das $r$ ist „,hiatustilgend“", wie 80 häufig im Baskischen; vgl. loquerẽ bei Voltoire (1642) für luqueen $\mathrm{S}$. $146 \mathrm{f}$.

4) Umstellung von Buchstaben:

a) unmittelbar nebeneinander stehender : at:

diadigula $\mathrm{DI}^{*} 17$. 


\section{LXVIII}

$$
\text { ce : }
$$

$R E$-|ecbilu Abc H VII ${ }^{*} 4$.

eu:

cueróc $288^{\circ} 14$ (unmittelbar

nach guec).

$$
\text { gn: }
$$

Jtang 455 r20 (franz.).

$$
\text { ic: }
$$

Guehienci ã VII $^{1}$ c 24.

Miferciordia i VII $^{\text {r b } 43 .}$

ih:

ohiu $56^{1} 23$.

it :

tti. I II'b 25 .

$\boldsymbol{l t}$ :

eftatlzen $14^{r} 24$.

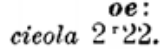

$$
\text { ft: }
$$

abratfeagatic ${ }^{* *}$ VIII $^{\mathrm{r}} 10$.

Vielleicht sprach L. so; vgl. abratfale $321^{1} 10$ und unter, ,zt". $t z$ :

baztu $372 \div 15$.

Es könnte eztitiát $434^{\circ} 2$ für das regelmässige etzitiat stehen; wahrscheinlicher aber ist mir ein Anderes; s. oben S. XXXVI. ze:

eçagutezco $\mathrm{CI}^{1} 16$.

$z t$ :

erautzen $354^{1} 23$.

$\mathrm{Zu}$ erauz $\mathrm{Gv}^{\mathrm{r}} 29$, erauciz $237^{1} 24$; vielleicht ist es aber für $\mathrm{L}$. die regelrechte Form, wie ikutzen zu ikuci, hertfen zu herffi, (,bedrücken" = erften zu ertfi ,schliessen“) gegenüber sonstigem erauzten, ikuzten, hersten.

vitzen $\widetilde{\mathrm{e}} \mathrm{III}^{1} \mathrm{~b} 27$. vizten $136^{1} 8$.

b) voneinander getrennter:

adiquifde $154^{1} 12$, adiquifdea adifquide(a). $184^{1} 11$.

baigunegui $\mathrm{D} \mathrm{II}^{1} 24$.

çaitzaizqueten $430^{\circ} 24$.

baguinegui.

çaitzaquezte $174^{1} 7, \quad$-tenari $290^{1} 25$.

S. oben S. LV. LXIV. Die Trennung des $z$ von dem $t$ durch das que vollzieht sich in der lab. Mundart welche Prinz Bonaparte im VB darstellt; man vergleiche: 


\title{
L.: çaitzaquezte dituqueizte çaituquezte
}

\author{
VB: gaitzazquete \\ dituzquete \\ saituzquete.
}

Aber man bemerke dass die der zweiten entsprechende Form mit der 3. S. als Urheber bei $\mathrm{L}$. dituque, im VB dituzque lautet, sodass dort $z+t$ als Pluralzeichen des Urhebers, hier $z$ als das des Subjekts, $t$ als das des Urhebers erscheint. şohian $113^{1} 2.18^{1} 42$ (vgl. *ciohan; cioacenéc $83^{1} 9$, cioabizc. joilan).

eyarthu $23^{1} 6.78^{1} 18$. eyarrha $64^{\mathrm{r}} \ddot{\mathrm{U}}$. işaganatic $\mathrm{B}^{\mathrm{r}} 3 \overline{5}$. prometattu $30^{\prime} \mathrm{U}$. Thabita $223^{1} \mathrm{U}$.

Man beachte noch:

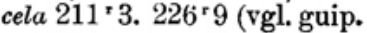
cioan).

eyhartu 40 r $20.84^{\text {r'2 }} 2$. eyharra $111^{-8} 8$. içanagatic. promettatu.

Tabitha $225^{1} 36.40$ (vgl. franz. 77 Thabita V. 36, Tabilha V. 40$)$.

baguetaco $350^{\circ} 15$ (bague ge- gabetucouc $376^{1} 23$; gabe immer. hört dem Süden an). bidebaguezcoa $\mathrm{Bv}^{1} 34$.

Venn L. regelmässig, wie es scheint: diarocat 13 г9. $368^{\circ} 12$. diraucagu, diraucac u. s. w. $428^{\circ} 9.433^{\circ}=1 .{ }^{12} 23$.

schreibt, so vermag ich das ebenso wenig zu erklären wie van Eys Gr. S. 336, bemerke aber dass jenes nach meiner Auffassung die ältere Form ist, für d-i-a-du-ca-t.

Während die bisher aufgezählten mundartlichen Varianten sich in ihrem Aeussern von den wirklichen Druckfehlern nicht unterscheiden, und manche derselben sich von diesen nicht mit völliger Sicherheit abtrennen lassen, gibt es eine Anzahl anderer die aus dem Kreise der Druckfehler ganz herausfallen, bei denen es sich nämlich nicht um einzelne an sich bedeutungslose Laute oder Silben, sondern um Worttheile mit bestimmter Funkzion handelt. Vor Allem in den Konjugazionsformen. Zunächst sehen wir Gleichwerthiges miteinander vertauscht. Für ,sein" gelten als Stämme ça und $a$, und sie werden sogar innerhalb derselben Flexionsgruppen verwendet, wobei zum Theil die Mundarten verschiedene Wege gehen. Besonders mannigfach gestaltet sich das Verhältniss zwischen "wir sind“ und "Ihr seid" mit dem Dativ (s. Bask. Stud. I, 36 oben, wo übrigens „(Liç.) zait" zu streichen ist). L. bildet die letzteren Formen, so viel ich sehe, nur von $a$; die ersteren bald von $\varsigma a$, bald von $a$ : 


\section{$\operatorname{Lxx}$}

gaitzaizquic $37 \cdot 27.81^{1} 28$. $143^{2} 28$.

ezgaitzaitza $399 \cdot 9$, ezquaitzaitza EvII' 38, gaitzaitza

* VII' 19. Erv' 23.

gaitzaizle $295^{1} 9$, ezquitzaizte

gaizquionac 274 × 2 , guiaizquio $320 \cdot 13$ u. s. w. (s. unten S. LXXII). $332^{\prime} 5$.

Höchst bemerkenswerth ist das schon oben S. XXXVI verzeichnete:

daicula $\mathrm{C}$ vi 17 .

saicu, z. B. C II'3. 10.

An der ersteren Stelle heisst es: ${ }_{n}$ dass die Thür die früher verschlossen uns war (faicun), jetzt geöffnet uns ist (daicu-)". L. pflegt den Unterschied 2 wischen dem Imperfekt und Präsens des zielenden işan nicht wie in andern Fällen am inlautenden Vokal auszudrücken (vgl. soul. ceicun, qaicu), und auch nicht durch die Vorsilbe ci- (die Form citzaicun hat van Eys VAL S. 33 unrichtig aus balitzaicu erzchlossen), sondern nur durch das auslautende $n$ : şayon, şayo - saizten, şaizte, also in manchen Fällen gar nicht. Das obige saicun kann an sich eben so wohl als saicu- $n$ wie als gaicun-n gefasst werden; da es nun aber dort darauf ankan Imperfekt und Präsens deutlich zu scheiden, so hat wohl L. zu einer mundartlichen, sonst allerdings nicht direkt belegten Form des letzteren gegriffen. Es würde sich caicu mit dem anlautenden Konsonanten an die entsprechende Form des transitiren Hülfsverbs (in cispyr. Mdd. daucu, daicu, bei L. draucu) angelehnt haben, wie es das mit dem inlautenden Diphthongen in niedernav. saucu gethan hat (s. Bask. Stud. I, t8). Lnd wenn wir auch nicht dies gaucu selbst bei L. haben, so doch das dazu gehörige sauc ( $\sim$ dauc), nämlich:

çarán, s. oben S. XLI, statt şayin.

Damit hängt dann wieder şaue in:

gauenac ebend.

zusammen, welches im Niedernavarrischen allerdings nicht vorkommt; eine Analogie dazu bietet nur das weit entfernte roncal. cabei $\sim$ trans. dabei (aber salaz. caye: dabe). - Man könnte daran denken in daicula die transitive Form selbst zu erblicken: ${ }_{n}$ er uns hat ${ }^{\mu}$; aber der Zusammenhang und der Wortlaut der französischen Vorlage lassen das kaum $z \mathrm{u}$, auch wäre die Entlehnung einer Form die von der bei L. herrschenden so abliegt, nicht weniger befremdend. Das intransitiv gefasste daicu stützt sich gegenseitig mit:

baitaucu $\mathrm{Abc} \mathrm{Bv}^{2} 10$,

welches $\mathrm{ja}$ an sich eine mundartliche Nebenform von baitraucu 
ist. Allein dem Sinne nach ist dies nicht am Platz; als transitive Form wäre baitraucuc zu erwarten, und so ist es vorzuziehen dieses baitaucu intransitiv, d. h. = baitzaicu zu nehmen. Und den beiden hier besprochenen Formen wird nun endlich auch anzuschliessen sein:

baitaye $429^{\prime} 13$ für baitzaye,

in dem ich anfangs mit van Eys einen Druckfehler zu setzen geneigt war, nicht mit dem Prinzen Bonaparte eine gesprochene Form.

Es muss hier darauf aufmerksam gemacht werden dass die mit -te oder -teque einerseits vom Stamme di, anderseits vom Stamme " gebildeten Flexionsgruppen ursprïnglich und auch heute noch verschiedene Bedeutungen haben (Potenzial - Futur), aber schon früh leicht miteinander vermischt werden; so sind bei L. nach ahal und ecin miteinander gleichwerthig:

daite $7^{\mathrm{r}} 14.163^{1} 4.5 .277^{\circ} 7$. date $135^{\prime} 26.27 .161^{\mathrm{Y}} 46.279^{\mathrm{r}} 6$. Das Pluralzeichen für das Subjekt + Dativzeichen hat bald die Gestalt $t z$ bald die: $z q u i$, $z c$; so:

$$
\text { baitzaitza } 413^{\prime \prime 2} \text {.2. } \quad \text { etzaizcala } 31^{1} 18 \text {, çaizquio }
$$

$293^{1} 14$, etzaizquionéz $\mathrm{i} \mathrm{VII}^{ } \mathrm{a}$

çaquitzate 406 ' 8 , gaquitzoncat ${ }^{* *} \mathrm{vi}^{12} 28$, bagaquitzu *** III $^{1} 1$.

drautza $5^{\prime} 8$, -
28 , buzaizquio E II ${ }^{1} 13$. saquizquiotc $7 \mathrm{~S}^{\circ} 7$, daquizquión $32{ }^{r} 11$.

druzea 169 20, drouzquionari 312 r28.

\section{(weitere Beispiele bei van Eys VAL S. 46.)}

Manche Formen scheinen nur mit $t z$ rorzukommen, wie carreitzate. Auch als blosses Pluralzeichen für das Subjekt wechselt Beides miteinander; der transitive Imperativ wird gewöhnlich mit itzac, itzague gebildet (so erraintzac $\mathrm{Abc} \mathrm{H}_{\mathrm{vn}}{ }^{\prime} 30=$ erran itzac), aber L. hat doch auch: emainzquiçue $364^{\prime} 18$ ( $=$ eman izq.).

Neben dadutza u. s. w. kommt, so viel ich sehe, daduzca u. s. w., welches sonst neit und breit herrscht, bei L. nicht vor. Einer eigenthümlichen Form dieses Verbs gedenke ich hier nebenbei; "er hält ihn ihnen" sollte, so erwarten wir, *diaducate (guip. dancaye) lauten, aber L. hat diadutela $\mathrm{C}_{\mathrm{II}}{ }^{\prime} 19$, wo also das -qui von eduqui sich gar nicht findet; die Form ist mit draue, lab. guip. diote materiell gleich, und ebenso * dadu, welches nach Ausschaltung der Dativelemente übrig bleibt, "er hält" mit bizc. dau, guip. lab. $d u$ "er hat".

Ferner gibt es quantitative Varianten. Ein pleonastisches Pluralzeichen für das Subjekt erscheint in : 


\section{LXXII}

baicurade $\mathrm{FVIII}^{\circ} 9$.

baiquinaden $247^{\prime} 8 . \mathrm{Av}^{\prime} 15$, guinadelaric $326^{\mathrm{r}} 15 \mathrm{~F}$. F

II ${ }^{\mathrm{r}} 35$, baguinade $325^{\prime} 2$. 327'21 (van Eys VAL S. 31 stellt guincude und lirade fälschlich $\mathrm{zu}$ den -te-Formen).

caiteztez $312^{\circ} 34.330^{\prime} 11$, ezpasaiteztez $236^{\prime} 1$.

çaquiztez $216^{1} 38,-\dot{e} z 401^{\circ} 17$.

baçaituztez $327^{120}$ (drei M.) (saituztéz $358^{1} 9.10$ ist $=$ nsie haben euch").

badaudez $298^{1} 8$, daudecẽçat 400 '27.

Ein pleonastisches Dativzeichen (s. Bask. Stud. I, 57 f.) in : guiaizquio (-onac, -onçat). gaizquionac 274.2.

$217^{\circ} 4 . \quad 320^{\circ} 13.385^{\circ} 3$.

$396^{\mathrm{r}} 39.411^{1} 24 . \mathrm{A} \mathrm{v}^{\mathrm{r}} \mathrm{j}$.

G vir' 6 .

Es geht nicht wohl an, den Anlaut aus der entsprechenden Dutzform guiaizquioc $\mathrm{CIV}^{1} 37$ abzuleiten; auch wäre an Stelle dieses ein gaizquioc möglich, vgl.: eznitzayoc $260^{\prime} 19$.

Unterdrückt ist das Dativzeichen in :

drauztán 1976 (soul. deiz- drauzquidán 1977. 8 (lab. tan).

cequizten $72^{\mathrm{r}} 33$.

Die erstere Form stimmt zu $216^{1} 38$. 401' 17 , caquiztençat $411^{1} 21$.

Wenn wir ein vereinzeltes cerezten $210^{1} 45$

finden, so ist das von cerauzten (etzerauztela $320^{1} 19$; Praes. drauzte) nur mundartlich unterschieden, und zwar durch die (dynamische) Wahrung des Dativzeichens (die Form çaizteten wird noch gebraucht, ostniedernav. cezteeten). Van Eys VAL S. 48 verzeichnet es als Dutzform, was es nicht ist, am wenigsten zu cerauccen, das er hier wie drauece (auch dirauc̈agu) irrig als ,les à eux..." erklärt (es ist, „le à eux ..."). Er ist dazu durch die Dutzform dirauecé $443^{\mathrm{r}} 10$ verführt worden, in der allerdings ,les à eux" steckt; sie gehört zur bestimmten Form drauzte (so $303^{1} 20$ ), welche van Eys für einen Irrthum statt drauece hält.

baicara Fir11; u. s. w.

aiquinen, guinen, bagu:na

regelm.

caquiztençat $411^{1} 21$.

caituzte $60^{1} 8$; u. s. w.

dauzquidan). cequizquien $208^{*} 3$ (für*-qui-te-).
aquiztén $287^{\mathrm{r}} 19$, çaquiztez, -éz 


\section{LXXIII}

Doppelt gesetzt ist das Dativpronomen der 3. P. (s. Bask. Stud. I, 56) in:

cieçoyon $44^{\mathrm{r}} 46$.

dietzoyoten $41^{1} 41$.

cieçon regelm.

cietzoten regelm.

Hingegen fehlt es, offenbar indem das stammhafte $o$ missdeutet wird, wie in guip. diago (Larramendi unter ,pertenecer"; s. Bask. Stud. I, 57), so in:

dagoten $289^{\mathrm{r}} 2.369^{\mathrm{r}} 10$ für

dagocaten zu dagoca (mit

Plural des Subjekts:

dagozca, z. B. $380^{1} 1$ ).

Bei dieser Gelegenheit will ich bemerken dass wenn „es sei ihm" den Sinn hat von ,er habe es", der Dativ überhaupt nicht am Verb ausgedrückt zu werden pflegt: nicht çayola,

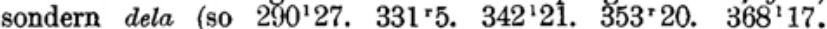
$379^{\circ} 18$. 419'18); hier liegt also nicht, wie man gemeint hat, ein Versehen vor. Das TB ${ }^{28}$ hat nur an einer dieser Stellen içan baiquio, an den übrigen $i_{c} a n$ bedi, was nicht gerechtfertigt zu sein scheint, da ja hier der Imperativ als Hülfsverb auftritt. Vgl. eztén (nicht eztaquión) $337^{\mp} 13$.

Ich erwähne hier noch die pluralischen Formen von etzan "liegen", welche nach Larramendi und Lardizabal $a u(e u)$ statt $a(e)$ haben, bei L. aber ausserdem ein schwer erklärliches $n$ : datza, cetzan - daunça, ceunçan. Dasselbe ist in der 1. und 2. Pl. Impf. allgemein und begründet: gueunçan wie gueunden (von egon); solite es von da ins Praesens und zugleich in die 3. Pl. eingedrungen sein? Ganz besondere Schwierigkeiten bereiten gewisse imperativische und konjunktivische Formen zu eman ,geben", welche auf einen andern Stamm zurückzugehen scheinen. Seine Stelle nimmt grossentheils ein -i- ein, in dem wir das Dativzeichen vermuthen könnten; dann läge es nahe den geschwundenen Stamm für den gleichen wie den von „haben“ zu halten; eztidala $339^{\circ} 17$, lidan $442^{1} 8$, iguc $9^{1} 11$, ligungat ${ }^{* *} \operatorname{vIII}^{*} 16$, diçuela $287^{1} 5$. $325^{r} 10$, diçuençát $340^{\mathrm{r}} 17 \mathrm{u}$. s. w. stimmen in der That zu guip. dit, digu, diçue, hier könnte man egun on diçula Iaincoac übersetzen: "guten Tag habe Euch Gott". Anderseits nähern sich die Formen mit der 3. Pl. als Dativ, wie eyec $72^{1} 36.240^{1} 35$, ey-, eieçue $27^{\prime} 16.88^{\circ} 9.248^{\prime} 28.351^{\prime} 2$, ezteyeçuela $11^{\prime} 6$, deyegunçát $160^{1} 22$, deyençát $131^{\text {"}} 42$ den soul. deyec, deyeçu, deyegu, deye von "haben". Vinson Rev. de ling. XXIX, $214 \mathrm{f}$. denkt an eguin "machen", und in der That liegen yquedac "du wirst mir ihn geben" und eyquec ,du wirst ihn machen", wie in den Sprichwörtern von $1596 \mathrm{zu}$ lesen ist, nicht weit auseinander. Aber die Bedeutungen 


\section{LXXIV}

stimmen weniger gut zusammen, - man könnte vielleicht daran erinnern dass der Baske für ,einen Kuss geben" lieber sagt pot eguin als pot eman, aber das ist ein ganz besonderer Fall. Mir scheint als ob das ieyncoac drugaçula bei Dechepare EIV' 1 in Berücksichtigung gezogen werden müsse; Stempf hat es durchaus missverstanden, es wird aufgeklärt durch Iaincoa trugatçula (trogatcula) „Dieu vous gard“ bei Voltoire (1642) S. 243. 278. Wie dem auch sein mag, mit dem -id- ,geb- mir" der obigen Formen lässt sich das ind- des Imperativs: indan $165^{17}$. 10, indac 166'15 schwer vereinigen, welches sich in allen Mdd., und zwar von den frühesten Denkmälern an findet. Voltoire hat zwar idaçu S. 236, aber da es hier von Druckfehlern wimmelt, so ist darauf nicht fest $\mathrm{zu}$ bauen. Vinson a. a. $\mathrm{O}$. setzt für iguçu an: (egu)inguçu, aber wie kommt das -n des Partizips in den Imperativ, d. h. in den einfachen (vgl. guip. eguiguçu) ? Der zusammengesetzte eguin eģaguçu lässt sich hier doch nicht wohl zu Grunde legen. Stehen nun hiermit die beiden Formen bailinguzquet „er wird mir sie (PJur.) geben“ $54^{\circ} 53$ und eztinguzteçuen, ,dass ihr sie (Plur.) ihnen nicht

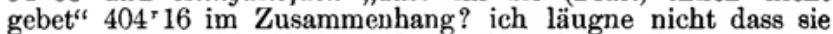
mir durchaus räthselhaft sind. Rathlos fühle ich mich auch dem ianagaçue $304^{\text {× }} 25.27$ für sonstiges ian eçaçue (z. B. 305²4) gegenüber.

Ausserhalb der Konjugazion findet sich der auffälligste Nebeneinandergebrauch mundartlicher Formen bei den Possessivpronomen, und zwar nicht bloss in der Sprache L.s, sondern in der vieler andern Schriftsteller verschiedener Zeiten und Gegenden, von Dechepare angefangen. Für die 1. und 2. P. Pl. hat L., so viel ich sehe, nur gure und şure, nicht auch, von gueuroc, ceuroc u. 8. w. abgesehen, gueure und ceure (vgl. z. B. Axular ${ }^{2}$ S. 19, 2: ,suc ceure moldera, eta çure herrian"), aber für die 1. und 2. P. S. gebraucht er ene und neure, hire und eure mit ausserordentlicher Willkür. Ich sage nicht, mit völliger; denn es werden Verbindungen nachzuweisen sein in denen die eine Form vorwiegt oder allein herrscht, vielleicht z. B. hic eure, nicht hic hire. Auch pflegen in den Parallelstellen dieselben Formen $\mathrm{zu}$ stehen; so in den sonst etwas voneinander abweichenden Fassungen des Vaterunsers immer hire. So Matth. VII, 3.4. 5: eure anayeren, eure beguico, eure anayeri, hire beguitic, hire beguian, eure beguitic, eure anayeren $=$ Luk. VI, 41. 42: eure anayeren, eure beguico, eure anayeri, hire beguian, hire beguian, eure beguitic, eure anayeren; so Matth. VIII, 8. 9: ene atharbean, ene muthilla, neure azpico, neure cerbitzariari $=$ Luk. VII, 6. 7. 8: ene atharbe pean, 


\section{LXXV}

ene muthilla, neure meneco, neure cerbitzariari. Aber innerhalb dieser Stellen vermögen wir kein Gesetz zu erkennen; ja, nicht selten zeigt sich in ganz gleichen Verbindungen eine fast regelmässige Abwechselung, z. B. Iefus Chrilt eure Seme $\mathrm{AI}^{1} 10 .{ }^{3}$. II $^{1} 12$. IV ${ }^{1} 10$, Iefus Christ hire Seme $\mathrm{AI}^{12}$ 20. II ${ }^{\mathrm{r}} 21$. III' 16. 27. IV ${ }^{\mathrm{r}}$ 9. Diese Possessivpronomen sind eigentlich Genetive, denen nur der Auslaut $-n$ abhanden gekommen ist, ebenso wie denen der 1. und 2. P. Pl., gure, sure, und dem der reflexiven 3 . P., bere. Im Nordosten gilt bere für beide Zahlen: „sein" und „ihr", im Südwesten nur für den Singular, für den Plural meistens beren. Van Eys Gr. S. 99 bemerkt: „On se serait plutôt attendu à une seule forme dans les dialectes basques espagnols, puisque la langue espagnole dit su pour ,son' et ,leur'." Er hätte hinzusetzen können dass während die französisch-baskischen Mdd., wenigstens die soulischc, hierin am wenigsten zum Französischen, auch dem Südfranzösischen stimmen, die am weitesten davon entfernte bizcaische am meisten dazu stimmt: bere "sein", euren "ihr" $\sim$ son, leur. Indessen entspricht doch euren (eureen) strenggenommen nicht einem illorum, sondern einem ipsorum, es ist der Genetiv von eurak "sie selbst". Und so ist nun beren, oder genauer berén der Genetiv von berac "sie selbst", also nicht eine lautliche Variante von bere ,sein". Von den labourdischen Texten setzen für ,ihr" (Pl.) die einen bere, die andern beren. Z. B. jenes das Evangelium von Haraneder (BB 268), dieses das von Duvoisin (Ausg. von Haristoy 1898), sowie das TB ${ }^{28}$. Bei L. nun ist zwar bere die herrschende Form; es findet sich aber auch keineswegs selten beren, so berén fynagoguetan $26^{\circ} 54$, berén ogui $72^{1} 36$, berén abillamenduac $83^{1} 7$, berén Iainco $98^{\circ} 16$ u. s. w. In dem letzten der angeführten Beispiele bezieht es sich sogar auf einen grammatischen Singular, der freilich pluralischen Sinn hat (anhitz....dic); im $\mathrm{TB}^{28}$ steht hier merkwürdigerweise bere neben dem grammatischen Plural (hainitz.... ditu). Manchmal folgen berén und bere oder bere und berén unmittelbar aufeinander, so: berén bihotzetaco guthicietara .... bere gorputz propriey $267^{\prime} 24$, bere bihotzetan, berén confcientiác $269^{\circ} 15$. - Auch nominale Suffixe treten zuweilen in mundartlicher Verschiedenheit auf. Dem lab. -taffun entspricht niedernav. soul. -tarçun; das letztere erscheint vereinzelt bei $L_{\text {. }}$ so neben dem gewöhnlichen eritaffun zwar nicht das niedernav. soul. eritarçun, aber doch erharçun $61^{\prime} 34$, -nac $168^{\circ} 5$ (unmittelbar vorher, V. 4 eritaffunec), welches sich in den westniedernav. Pregariac von 1651 S. 12 findet, und wofür van Er̄s als lab. verzeichnet erhasun; vgl. ontaffun "Güte", 


\section{LXXVI}

onhaJfun "Gut" (= „Besitz") bei L., sowie offaffun a mi'b 1. . Ferner lesen wir fabeldarşunac $264^{1} 8$; auch Larramendi hat unter "disenteria" merkwürdigerweise - als ob sie auch transpyrenäisch wäre - die Form sabeldarguna (auch sabeldarçunez dagoana). - Unter den Verben begegnen uns nicht nur solche mit verschiedener Ableitung als gleichwerthig, sondern auch das abgeleitete und das einfache. Eine interessante Form ist deitha $422^{\prime} 1$. Ich dachte zuerst an einen Druckfehler; aber deithu, wie Dodgson The Basque Verb found and defined I, 23 lesen will, geht nicht an; neben gaitecen hat nicht das Partizip, sondern das Radikal zu stehen (z. B. etzaiteztela dei $44^{1} 10$ ). Deitha als Druckfehler für dei lässt sich wiederum kaum erklären. Deithatzen war mir sonst unbekannt, bis auf das von Larramendi verzeichnete deitatua "famoso". Nun aber lese ich in den westniedernav. Pregariac von 1651 S. 10 bere gana deithatu ,zu sich gerufen“; hierzu gehört jenes deitha L.8 als Radikal. - Neben gabe findet sich, aber unter hundert Fällen kaum zwei Mal, gaberic $369^{\mathrm{r}} 8.393^{\mathrm{r}} 7$, häufiger gaberico neben gabeco, und zwar ganz in den gleichen Verbindungen, so:

altchagarri gaberico $52^{\prime} 17$. altchagarri gabeco $90^{\circ} 12$. $150^{1} 1 . \quad 151^{\mathrm{r}} 7.230^{\mathrm{r}} 3$. $247^{1} 6.296^{\prime} 8,-$ coen $89^{\prime} 1$.

hypocryfia gaberico $321^{\circ} 6$, fictione gabeco $374_{j}^{1} 5$. fictione gaberico $368^{\mathrm{r}} 5$.

(auch gabetaco kommt vor; s. oben S. LXIX.)

Aehnliche Verhältnisse finden sich öfter; vgl. z. B.:

bekatuzco atjeguinén $397^{1} 25$. Atfeguin bekatutacoez ẽ $\mathrm{II}^{\mathrm{r}} \mathrm{a}$ 36 (bezieht sich auf die andere Stelle).

hirazco mahatfarno $454^{\prime} 15$. hirataco mahatfarnoac 447 r8. hiraco mahatfarnotic $447^{\prime} 10$.

bihotzezco tauletan ** VII ${ }^{1} 34$. bihotzeco taula $317^{1} 3$. bihotzezco fimplicitatere- bihotzeco fimplicitaterequin quin $210^{1} 46$. $357^{1} 22$.

Die grösste Menge von Varianten weisen die Fremdwörter auf; das ist natürlich, sie sind aus verschiedenen Quellen geschöpft, aus lateinischer, schriftfranzösischer, südfranzösischer, spanischer, und dann wiederum in verschiedenem Grade baskisiert. Dafür sind schon oben genügende Beispiele gegeben worden; hier folgt noch einiges Ergänzende und Erläuternde. In Bezug auf die Schreibung der ganz fremden Eigennamen herrscht kein festes Prinzip; auch die gleichen werden bald so bald so geschrieben, z. B.: 


\section{LXXVII}

Publiufen $264^{\circ}$ Ü.

Antiochian $377^{\prime} 11$.

Corinthion $243^{\mathrm{r}} \mathrm{U}$.
Publioren $264^{1} 8$.

Antiochen $229^{1} \mathrm{C} 0$; u. s. w.

Corinthen $245^{\mathrm{r}} 1$; u. s. w.

Im TB ${ }^{28}$ ist die Buntheit auf die Spitze getrieben; wir finden z. B. zwar immer Corinthia, Antioche, aber Sardea(n), Sardas(en), Sarda(co) - Ephese(rat), -(tic), Ephesea(n), Ephesia(rat), -(n), -(co), Ephesio(rat). Die mundartlichen Gestaltungen der Suffixe vertheilen sich meistens in fester, wenn auch nicht immer leicht erklärlicher Weise auf die einzelnen Stämme, so wird z. B. regelmässig heredero, aber officier, prefoner u. s. w. geschrieben; vgl. oben S. XXXII -antia, -ancia, (-ança) und -itia, -etia, -ecia, - eşa. Von den unwesentlicheren Varianten abgesehen, haben wir auch solche wie:

benediçoin $339^{\prime} 3$.

benedictione $389^{1} 7$ u. s. w., jenes nach Analogie vollständig eingebürgerter Wörter wie cantoin, raçoin u. s. w. Ich schalte hier die Bemerkung ein dass auch bei romanischem Stamm und romanischem Suffix das baskische Wort nicht immer seine Entsprechung im Romanischen hat. Die Basken verwenden die romanischen Suffixe vielfach in selbständiger Weise, und sind sogar geneigt schon vorhandene romanische Ableitungen von Neuem zu bilden; vgl. fermutate für fermetate oben S. LI und erretate für *erreguetate (gegenüber romanischem regalitate), welches bei $\mathrm{L}$. allerdings nur als soulisches Wort mit verändertem Endvokal: retatuia ã VII ${ }^{1}$ c 40 vorkommt. Da die Mozion dem Baskischen fremd ist, so werden, wo sie sich schwer ersetzen lässt, die romanischen Wörter mit ihrer doppelten Endung beibehalten:

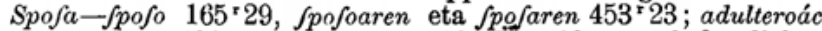
eta adulteríc $406^{\mathrm{r}} 4$; Samaritanari $165^{\mathrm{r}} \mathrm{U}$. Aber noch deutlicher wird das weibliche Geschlecht für den Basken durch die romanische Endung (i)/ja: bekatore $\int \rho a 113^{1} \ddot{U}$., di/cipuluffa $225^{1} 36$, beim Adjektiv : fidelec - fidelfac $372^{\mathrm{r}} 16$, und bei einem baskischen Wort: iainco/fä 247 r 37 . Das auch bei Larramendi verzeichnete vitança $\left(48^{\prime} 4 \overline{5}\right.$. $\left.373^{r} 8.404^{r} 15\right)$ scheint aus span. vito $=$ victo neu abgeleitet zu sein. Auch im Stamme der Fremdwörter zeigen sich hier und da stärkere Varianten, so:

Excommicu ẽ VIII ${ }^{1} \mathrm{~b} 1$ (auch Excommunicatione ẽ $\mathrm{VIII}^{1} \mathrm{~b} 3$.

heute escomicatu), -icatzẽ B III'13.

ingea $-37^{\circ} 28.115^{\prime} 43$ u.s.w. iudica- $11^{\prime} 1,112^{\prime} 37$ u. s. w. An das zweite Variantenpaar schliesst sich unmittelbar an, freilich mit Endungen verschiedenen Ursprungs:

iugemendu- $11^{\ulcorner} 2.21^{1} 18.20$ iudicio- $7^{\prime} 22.17^{\prime} 15.20^{\circ} 22.24$

u. s. W.

u. $\mathrm{s}$. W. 


\section{LXXVIII}

Solche Synonymen deren etymologischer Zusammenhang mehr oder weniger verdunkelt ist, führen unmerklich zu solchen Synonymen hinüber bei denen gar keiner besteht. Eine Synonymik L.8, an welche erst nach der Herstellung einer vollständigen Konkordanz gedacht werden kann, wird zu prüfen haben inwiefern das was wir Synonymen nennen, mögen es nun Wörter oder Wortformen sein, wirklich unter ganz gleichen Bedingungen miteinander vertauschbar sind oder ob sie nur innerhalb eines bestimmten Kreises sich decken und worin dann der theilweise Unterschied zwischen ihnen besteht and ob nicht auch gelegentlich dieser Unterschied vernachlässigt wird. Es scheint sich das grundsätzliche Verhalten im Lauf der Uebersetzungsarbeit mehrfach geändert zu haben. Bei diesen Untersuchungen muss uns der griechische, der lateinische und vor Allem der französische Text auf Schritt und Tritt begleiten. Die Parallelstellen verdienen besondere Beachtung; aber auch die Ausdrücke des Sachverzeichnisses (ã ff.) müssen mit denen des N.T. selbst verglichen werden; so entspricht z. B. dort $\mathrm{iv}^{\mathrm{r}} \mathrm{b} 20$ haztamuca (von haztatzen, wohl \} lat. tastare) dem manu tentans der Vulgata von Pet. II, I, 9, während das N.T. selbst $416^{\circ} 9$ deus vrrundanic eztacuffalaric $=$ ne voit goutte de loin $=\mu \nu \omega \pi \alpha ́ \zeta \omega v$ bat. Im Vaterunser steht $9^{1} 13$. 126r4. BII'22 gaichtotic im Einklang mit franz. ${ }^{63} d u$ malin, aber $\mathbf{A} \mathbf{V I}^{1} 30 . \mathbf{A b c} \mathbf{A ~ I V}^{1} 13$ gaitzetic $=a$ malo als Neutrum $=$ franz. ${ }^{~}$ du mal; die Randbemerkungen lassen Beides $\mathrm{zu}$ : ,ou, du mal, c. de peché, ou, du mefchant, du diable", "ou, mefchant, ou mal". Auf dem Titel des Abc zitiert L. Matth. XIX,14 nicht ganz so wie er im N.T. selbst übersetzt hatte; er sagt haourrac $=$ haourtchoac, horrelacoén $=$ hunelacoén. Dodgson, indem er annimmt, L. habe sich nur an den französischen Text gehalten, kommt $\mathrm{zu}$ manchen falschen Behauptungen. So sagt er The Basque Verb found and defined I, 16, L. habe Joh. I, II, $25\left(422^{r}\right)$ "vous" in "nous" geändert: draucun; aber

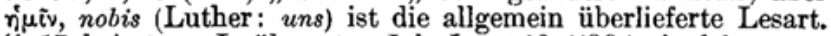
S. 17 heisst es, L. übersetze Joh. I, v, $10\left(426^{r}\right)$, le fait" statt "l'a fait"; die Vulgata aber hat facit (Luther: macht). Ebenso verhält es sich Joh. I, III, 1 (422'), wo nach Dodgson a. a. O. S. 24 L. ,infidèle" ist, wegen ezgaitu; vgl. niucus, nos: vous (Luther: euch). Ebend. II, 6 sagt er von Tim. II,III, 14 (377'): „Leicarraga ne serre pas le texte français" - wohl aber den griechischen und lateinischen. Auch zeigt sich Dodgson mit dem franz. Sprachgebrauch des 16. Jhrhs. nicht vertraut: II, 7: „s'amusans ne traduit pas behatzen" $\left(370^{1} 1\right)$; II, 14 zu probetchua ( $377^{\circ}$ Ü.): "Leiçarraga ne traduit pas l'usage." In andern Fällen handelt 


\section{LXXIX}

es sich um ein Missverständniss des baskischen Sprachgebrauchs; so ist nach Dodgson a. a. O. II, 5 neben aquió $\left(371^{\mathrm{r}} 13\right)$ das franz. attentif unilbersetzt geblieben - aber aquió

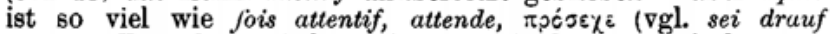
aus). - Zunächst würden wir uns mit den romanisch-romanischen Synonymen zu beschäftigen haben, welche häufiger sind als man erwarten sollte. $Z$. B. wird das lateinische mysterium durch Secretu und myfterio wiedergegeben; zuerst folgt L. dem französischen Texte, der überhaupt nur $2 \mathrm{Mal}$ myjtere, $15 \mathrm{Mal}$ fecret hat, indem er secretu schreibt: $24^{\circ} 11$.

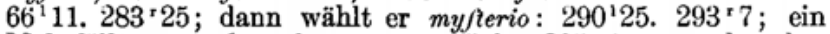
Mal fällt er noch auf fecretu zurück: $295^{\circ} 1$, von da aber bleibt er fest bei myfterio. Streng genommen haben wir hier ein griechisch-lateinisches Synonymenpaar, wie wir deren häufiger finden; man vergleiche noch hypocryfia gaberico $321^{\mp} 6$ und fictione gaberico $368^{r} 5$. Weit grösseres Interesse besitzen für uns die romanisch-baskischen Synonymen. Man begreift nicht recht wie $L$. seine Uebertragung mit so vielen romanischen Wörtern durchsäen konnte für die er an andern Stellen echtbaskische verwendete. Wenn bei ihm dem lat. confiteri, franz. confe/fer bald confe/fatzen, bald aithortzen entspricht, so scheint damit doch kaum eine Bedeutungsschattierung verbunden zu sein, mag auch vom Bekennen der Sünden nur ersteres gebraucht sein; beide Verben treten in den gleichen Verbindungen auf, und das confe/fatzen von $349^{1} 11$ wird im Sachregister $\tilde{e} I^{r}$ a 29 durch aithortzen ersetzt. Ganz ebenso wie in diesem Falle verhält es sich mit charpanter $26^{1} \overline{5}$. $70^{1} 3=$ çuharotz $\widetilde{\mathrm{e}} \mathrm{v}^{1} \mathrm{~b} 28$. Bei einem und dem andern romanischen Worte bedauern wir dass uns kein echtbaskisches Synonym dazu überliefert ist, so bei conciertoac $245^{1} 12=\sigma e \mu$ :xiv $`\left(\alpha\right.$, semicinctia, deuantiers (das $\mathrm{TB}^{28}$ hat loihalac); diesem liegt concertar im Sinne von ,zusammenfügen" oder von ,schmücken“ (so im älteren Spanisch; vgl. port. concerto "Schmuck") zu Grunde. Am Allerwichtigsten sind für uns natürlich die baskisch-baskischen Synonymen. Hier werden wir freilich meistens irgend einen Bedeutungsunterschied ermitteln können. Ikar eçaçue $72^{1} 38={ }^{\prime} \delta \varepsilon \tau \varepsilon$, videte, regardez scheint ganz dasselbe zu sein wie ikuffaçue, und dieses könnte auch hier stehen (so hat Duvoisin); aber ikartzen hat genau betrachtet eine engere Bedeutung, die von "nachschauen", und kommt anderswo, theilweis in der Form ikhertzen mit den Bedd. "untersuchen", „prüfen", „visitieren“, „(einen Kranken) behandeln" vor. Damit stimmt es aber wiederum gar nicht dass $58^{1} 3$ ikartze ganz so gebraucht ist wie sonst $i k u f i e$, nämlich im Sinne von "Antlitz". 


\section{$\operatorname{LxXX}$}

Wenn wir uns an die Vulgata halten, so sind or (or-a) und chakur Synonymen für canis; aber der griechische Text zeigt uns dass letzteres als Deminutiv gilt (was es ja auch seiner Form nach, neben cakur, von Hause aus ist, heute jedoch kaum mehr) - es vertritt xuvápıov, welches (im Pl.) im französischen T'exte nur an der ersten Stelle (Matth. XV, 26. 27) mit petis chiens wiedergegeben ist, an der zweiten (Mark. VII, 27.28) mit chiens schlechtweg. Das ninicatzen (vielleicht romanischen Ursprungs) von $48^{\mathrm{r}} 32$ neben dem vftertzen von $89^{\mathrm{r}} 28$ beruht auf dem franz. eftre en feue neben eftre tendre, welches an beiden

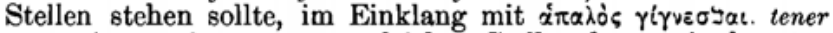
esse. An zwei sonst ganz gleichen Stellen lesen wir das eine Mal 24'15 gogorqui, das andere Mal 265'27 gothorqui,,schwer" „hart" vom Gehör; Beides ist möglich, und doch möchte man dieses in jenes verbessern.

Neben den Formen und Wörtern gleicher Bedeutung oder wenn ich so sagen darf, den morphologischen und lexikalischen Synonymen gibt es nun drittens syntaktische Synonymen. Es sind dies ebenfalls Formen, nämlich solche von verschiedener Funkzion, welche nicht im Allgemeinen, sondern nur in gewissen Verbindungen miteinander vertauscht werden dürfen oder wenigstens gelegentlich, durch Mundartenmischung vertauscht werden. Die der ersten Klasse sind, soweit sie nicht auf Lautveränderung beruhen, aus solchen der dritten hervorgegangen; was ursprünglich verschiedenwerthig war, indem es aus Elementen verschiedener Art oder $\mathrm{Zahl}$ bestand, ist gleichwerthig geworden, wie bere und beren, date und daite. Daher mögen sich in dem einen oder dem andern Fall bezüglich der Zuordnung Zweifel erheben. Dem ausgedehnten Gebiete der syntaktischen Vertanschungen entnehme ich zur Erläuterung nur wenig Beispiele, für welche die Grammatiken anderer Sprachen allgemeine Analogieen darbieten werden. Fast nirgends ist die gleiche Verwendung verschiedener Kasus unbekannt; so auch im Baskischen nicht. Gabe hat den Partitiv oder den Nominativ vor sich; bei L. meist den letzteren. Vgl.:

macularic gabea $391^{1} 26,-$ gabe $393^{\prime} 14$.

-. praulfuric gabe $360^{1} 13$.

reprochuric gabe $97^{1} 6$. macula gabea $400^{\prime} 4.403^{\mathrm{r}} 27$, - gabe $419^{r} 14$.

pauffiu gabe $266^{1} 9 . \quad 359^{1} 3$. $364^{r} 17.374^{1} 3$.

reprotchu gabe $360^{1} 10.362 \times 13$. $373^{\prime} 14.419^{\prime} 14$.

Guin hat den Genetiv oder den Nominativ vor sich; jenen meistens wenn der Artikel, diesen wenn er nicht steht: Se- 
mearen gainera - etche gainera, doch auch z. B. dorreac gainera $132^{\mathrm{r}} 4-$ Apolloren gainera $295^{\mathrm{r}} 6$. Wir haben hier sogar sehr eigenthümliche Genetive der unbestimmten Deklinazion nämlich auf -n statt auf -ren:

şuen burun gain $243^{\prime} 6$.

efcun gainean $257^{1} 26$ (vgl. niedernav. escungain

Ebenso in: ,avantmain"). haren buru gainera $51^{1} 7.89^{1} 3$, - gainean $56^{1} 29.203^{\mathrm{r}} 7$, bere buru gainera $285^{\mathrm{r}} 20$.

gogongaraitic ${ }^{* *} \mathrm{\Psi}^{\mathrm{r}} 15$.

gogoz garaitic $302^{\mathrm{r}} 17$,

da garai den Genetiv, Instrumental und Nominativ regiert. Beldur wird regelmässig mit dem Genetiv verbunden; ausnahmsweise mit dem Dativ: hari beldur cayona $227^{\prime} 35$ (auch $71^{\prime} 20$; s. Dodgson Rev. de ling. XXXI, 142). Das Futur wird von den Partizipen auf $i$ in der Mehrzahl der Mdd. mit -co (,lokaler Genetiv") abgeleitet, in manchen mit -en ("possessiver Genetiv"). L. begünstigt das Letztere; doch hat er ausnahmsweise jenes: ethorrico dirade $46^{\top} 36$, -ico ethorriren dirade, da u. s. w. da $47^{\times} 14$. $13^{\mathrm{r}} 11.15^{1} 15.32^{\prime} 11$ u. s. w.

Dieses ethorrico $d a$ ist nicht $z u$ verwechseln mit ethorteco da, das auch durch unser Futur wiedergegeben werden kann; man beachte: ethorteco dena ethorriren da $396^{\mathrm{r}} 37$ (qui venturus est, veniet $=$ qui doit venir viendra). Mancherlei Wechsel von minderer Bedeutung stösst uns noch auf; so übersetzt L. „s'apparut à luy par fonge" (in somnis, x $\alpha \tau^{\prime}$ "ovap): aguer cequion ametfetaric $2^{2} 20$, aguer cequión ametjetan $3^{1} 19$. Im Westlabourdischen (insbesondere zu Saint-Jean-de-Luz) herrscht eine ganz unbaskische Konstrukzion: ,schuldig sein" mit doppeltem Nominativ (im Sinne unseres Akkusativs), welche als Romanismus zu betrachten ist, indem man das enklitische Personalpronomen des Dativs für das des Akkusativs hielt. So hat schon Voltoire, der sich, wie er selbst sagt, an das Baskische von SaintJean-de-Luz hält: Eftutufté hay ñ berçe sorfeytudela „Ie ne pense pas vous deuoir tant" (S. 225), ebenso (BB 788) Pouvreau um dieselbe Zeit: Etçaitut hura çor (S. 25). Eine Spur davon findet sich bei L.: baldin cerbait . . . . sor bahau $383^{1} 18$; Dodgson "Euskara" S. 88 a bemerkt richtig dass es ģor badrauc heissen müsse; so steht ja in der That im folgenden Vers: niri çor drautadala. Ganz ähnlich verhält es sich mit eznauçe ihardetfiren $153^{\prime} 68$, wofür wir eztrautaçue $i$. erwarteten. Noch befremdlicher, und ebenfalls aus romanischem Einfluss zu erklären ist der doppelte Nominativ neben einem intransitiven 


\section{LXXXII}

Verb. Es handelt sich dabei nicht etwa um einen Prädikatsnominativ, sondern um einen Objektsnominativ, an dessen Stelle ein Dativ oder anderer Kasus zu erwarten wäre. Iruditzen "ähnlich sein" ist im Baskischen transitiv, so gewöhnlich auch bei L., und zwar das Präsens entweder dirudi oder in perfektischer Form irudi du; daneben aber auch, ohne dass die Konstrukzion geändert wird: irudi da, so nor irudi den $113^{\prime} 47$, iģanen naiz guec irudi $179^{\prime} 55$, anayeac irtdi licén $386^{\prime} 17$. Irudi da heisst eigentlich ,er ist ähnlich“, und es sollte neben dem Adjektiv der Genetiv (Semearen irudico $390^{\prime} 3$ ) oder der Direktiv (hetarát irudi 9'8) stehen: aber wie im Spanischen parecido nicht anders verbunden wird als parecer, so folgt nun auch irudi gelegentlich dem Verbum finitum. Wir könnten uns auch so ausdrücken: nor irudi den ist eine Vermischung von noren irudi den und nor irudi duen. Einen solchen Nominativ liebt neben sich auch das intransitive efcatzen "verlangen", mag die Person von der man etwas verlangt, genannt sein (im Dativ) oder nicht, z. B. arrain efca badaquió $11^{\prime} 10$, cer-ere efcaturen baitzarete $192^{\prime} 13$. Weit seltener kommt daneben der Genetiv vor, und zwar unter den gleichen Bedingungen : efca cequión ..... gorputzaren $95^{1} 43$, cerè-ere ...... efcaturen baitzarete $84^{12}$. Doch ist dies wohl das Ursprüngliche, und somit efca eigentlich ein Substantiv; dann wirkte das Romanische ein; der L.sche Gebrauch bildet nur den Uebergang zu dem der heutigen Mdd., in denen das Verb ganz transitiv ist. Bei bilha ebillen "Buchen gehen", bilha ethorten "suchen kommen" besteht ein "ähnliches Schwanken; der Genetiv erscheint dem Charakter der Sprache angemessen, so Noren bilha şabiltzate $1198^{\circ} 4 .^{\prime} 7$, aber häufiger ist fast, offenbar im Anschluss an bilhatzen, das auch bei L. transitiv ist, der Nominativ, so aicina bilha çabilan $52^{\prime} 16$. Ja, sogar die vom Verbalstamme gebildeten Instrumentale efquez und galdez (,verlangend") regieren ebenso gern den Nominativ als den Genetiv, z. B.:

$$
\begin{aligned}
& \text { figno efquez dago } 22^{\prime} 39 \text {. ceren efquez şaudeten } 38^{1} 22 \text {. } \\
& 30^{1} 4 \text {, figno .... esquez } \\
& \text { şaizcan } 126^{i} 16 . \\
& \text { figne galdez dago 76'12. fignoren .... galdez saizcala } \\
& 76^{r} 11 \text { adoraçalén galdez } \\
& \text { diagon } 166^{1} 23 \text {. }
\end{aligned}
$$

Auch ohne die angedeuteten Begleiterscheinungen ist die Scheidelinie zwischen transitiver und intransitiver Form, welche ja im Baskischen eine viel tiefere und festere ist als in unsern Sprachen, bald von der einen bald von der andern Seite her 
überschritten worden. Vor Allem ist ein solcher Wechsel bei behar und nahi auffällig. Beide Wörter sind eigentlich Substantive: „Nothwendigkeit" und "Willen". Behar du eman (oder eman behar du) bedeutet:" "er muss geben", behar da eman: „es ist nöthig zu geben", „man muss geben", und dann auch persönlich: „er muss gegeben werden" (wodurch noch keine $Z_{w}$ weideutigkeit veranlasst wird: „es ist nöthig den Ring zu geben" = „der Ring ist nöthig gegeben zu werden"), und dazu behar naiz eman: "ich muss gegeben werden" (behar dut eman „ich muss geben"). Das im Partizip stehende intransitive Verb wird zunächst mit dem transitiven Hülfsverb verbunden: behar du ioun; daraus wird durch Attrakzion: behar da ioan, welches auch bedeuten kann: "man muss gehen", während behar naiz ioan wieder eindeutig ist. L. nun kennt hier beide Verbindungsweisen: die erstere z. B. 31'21. 91'31. $144^{\circ} 5$, die letztere z. B. $32^{1} 10.149^{\circ} 9.163^{\prime} 7.362^{\circ} 1$. Anderseits greift bei ihm das transitive Hülfsverb in die Sphäre des intransitiven hinüber, indem es sich bei dem passiv gedachten Partizip einstellt. Behar nincén ... laudatu, ich musste gelobt werden" heisst es $329^{\circ} 11$; aber behar diat ..... batheyatu ,ich muss getauft werden“ $4^{1} 14$, behar du handitu ,er muss grösser gemacht werden" $165^{\mathrm{r}} 30$ (vgl. auch $\left.236^{1} 5.443^{\circ} 5\right)$, was ja auch bedeuten könnte: ,ich muss ihn taufen“, ,er muss ihn grösser machen". So entsteht denn eine Satzverknüpfung wie behar diradela flacuac fupportatu, eta Iefus Iaunaren hitzéz orhoit içan $249^{\mathrm{r}} 35$, wo zu dirade verschiedene Subjekte gehören (vgl. behar ditugu ..... flaccata/fiunac fupportatu $287^{\top} 1$ ). Bei nahi schwankt, soviel ich sehe, L. fast gar nicht; er sagt nahi $d a$ ioan, nur ganz ausnahmsweise $n$. $d u$., so:

nahi baitu .... ethorri ethorrinahibada 31'24.121'23. $77 \cdot 34$.

Verschiedene Verben werden, auch in ganz gleicher Bedeutung, transitiv und intransitiv konśtruiert, so:

bere buruä complacitu 287' 3 . garait badesa $127^{\circ} 22$, garait equa $285^{\circ} 21$, garaithuren citic $443^{\mathrm{r}} 7$, ezpaita garaithu içan $\mathrm{Cv}^{\mathrm{r}} 34$.

Macedonia iragan duquedanean $313^{1} 5$ (so regelmässig).

Das Radikal erscheint statt des Infinitivs neben dem Präsens : aithor dituzté $254^{\circ} 8$. gure buruey complacitu $287^{\prime} 1$. garaithu natzayo $196^{1} 33$, garaithu içan çayó $\mathrm{CIV}^{125}$; u. s. w.

Macedonian iraganen naiz $313^{1} 5$ (vgl. 239:4).

aithortzen baitu $422^{\mathrm{r}} 23$; u. s. w. 


\section{LXXXIV}

Der Konjunktiv des Imperfekts steht ein paar Mal für den Indikativ desselben Tempus:

lioitela $254^{1} 12$ (es folgt un- cioitela 4 r 29 ; u. s. w. mittelbar ezlutela). ezlaquiala $67^{\prime} 27$.

Etzaquiala 121'33; n. s. w.

Nach ez- beginnt die neue Seite; es kann sich hier also um irgend eine äusserliche Irrung handeln. Und auch in den folgenden Beispielen ist der Konjunktiv als das Unregelmässige $\mathrm{zu}$ betrachten, da in den indirekten Fragesätzen cen und çuen zu stehen pflegen:

nor licén, eta cer eguin şuen $251^{\prime} 33$.

cer herioz hil behar luen cer herioz hil behar çuen $188^{1} 33$, $199^{1} 32$.

c.h. glorificaturè çuen $205^{1} 19$ (an allen drei Stellen geht aditzera emaiten çuela vorher).

In befremdlicher Weise vertritt das Imperfekt das Perfekt in: hil cituán $147^{\prime} 31$.

hil ci(e)dián $147^{1} 30.32$.

Das hil içan duc des vorhergehenden Verses als die Erzählung einleitend begreift sich besser, und wiederum auch das ebendaselbst für das Imperfekt stehende içan dituc. In der entsprechenden Stelle 86 20 findet sich in der 'That das Imperfekt cituán (Dodgson Rev. de ling. XXXII, 70 wundert sich dass L. nicht baciraden schrieb, was er doch nicht schreiben konnte, da er sich der Dutzform bedienen musste - und verwechselt cituán mit cituen, sodass er L. den Vorwurf macht: ,il pense en erdara", d. h. auf Romanisch). Statt des Imperfekts setzt L. nicht selten das Präsens zum Theil im Anschluss an die Texte in den andern Sprachen (so besonders bei „sprechen"), zum Theil auf eigene Hand, so z. B.: dacarqueitela $109^{\prime} 18$. ekarten şutela $62^{1} 3$.

Beide Stellen sind inhaltlich gleich; doch mag das Präsens durch das vorhergehende huní begünstigt worden sein. Uebrigens ist dacarqueite eigentlich ein Futur (so ezpaitacarque $135^{\prime} \cdot 27$ in futurischem Sinne; in rein präsentischem dacarque Abc Hvirr '28). Wie in der heutigen sprache, so findet sich schon in der L.s bei diesem Verb das -que auch in präteritalen Formen, so in den Imperfekten çacarquela $200^{1} 5$. 201 ${ }^{\circ} 17$, çacurqueitela $250^{\mathrm{r}} 16$. - Gegen die Konkordanz verstossen: gaitz daritzuëney $8^{\prime} 44$, on on daritzoteney $278^{\circ} 27$. daritzueney $8^{1} 46$.

Sie würden buchstäblich übersetzt lauten: „eis qui vos odit - diligit". 


\section{LXXXV}

Insofern dem Sinne nach mehr oder weniger miteinander übereinstimmende Wörter oder Wortformen sich auch ganz ähnlich sehen, nur in einem Buchstaben voneinander abweichen, können sie von denselben Druckfehlern betroffen werden, wie ähnliche aber ganz sinnfremde Wörter (vgl. z. B. içan und icen oben S. LI). Das würden dann Buchstabendruckfehler sein, wenn sie auch durch die Aehnlichkeit der Wörter hervorgerufen worden wären. Wortdruckfehler rein mechanischer Art, wie die Verdrängung eines Wortes durch ein kurz vorher vorgekommenes, habe ich bei $L$. nicht entdeckt. Zu Wortdruckfehlern führt auch falsche Ausführung der Korrektur, indem zwei Schreibungen miteinander verschmolzen werden. Vielleicht liegt ein derartiger vor in norc cer ioan legaqueen 94'24; obwohl mir ein Baske versichert, es lasse sich so sagen, so verstehe ich doch die transitive Konstrukzion des intransitiven ioan nicht; ich möchte glauben, L. habe zuerst entweder norc cer eraman leçaqueen oder nori cer ioan lequion (vgl. nori cer erorico citçaioen bei Haraneder, und niri heltzen gaitadan partea $136^{\prime} 12$ ) geschrieben und das Eine in das Andere verbessern wollen. Noch einfacher allerdings scheint es anzunehmen dass eroan, welches die beiden ersten Buchstaben mit dem vorhergehenden Worte gemein gehabt hätte, in ioan verdruckt worden sei; aber obwohl eroan wie eraman dem franz. emporter ( $q u$ 'en emporteroit chacun) entspricht, so weiss ich doch nicht ob L. es mit Beziehung auf Kleidung8stücke gebraucht haben würde. Sicherlich aber steht manchmal bei L. ein falsches Wort oder eine falsche Form dank einem von ihm selbst begangenen sachlichen Missverständniss oder Verschen. So muss Capitainari $253^{\prime 25}$ durch Centenerari ersetzt werden, so deabruaren copá $303^{\prime} 21$ durch deabruén c., wie ja gleich darauf richtig deabruén mahainean steht. Irrigerweise hat $L$. die letzten Worte von $165^{1} 9$ ebenfalls der Samaritanerin in den Mund gelegt und eztié statt eztute geschrieben. Wollte ich diesen $\mathrm{Pfad}$ weiter verfolgen, so würde er mich mitten in eine sehr umständliche und schwierige Kritik des ganzen Uebersetzungswerkes führen. Ich bemerke hier nur so viel dass wenn L. zureilen recht frei übersetzt, das nicht immer geschieht ohne für uns eine Dunkelheit oder Schwierigkeit zu erzeugen welche in den Kreis der gegenwärtigen Betrachtungen gehört. Wir lesen $23 \bar{j}^{r} 3$ conflantqui minçatzen ciradela, Iaunaren

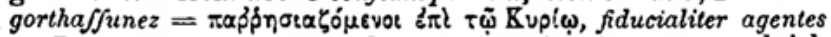
in Domino, se portans hardiment en noftre Seigneur, und ich wenigstens weiss mit dem letzten Worte Nichts anzufangen; denn gortaffun „Taubheit", gogortaffun „Härte", gorataffun. 


\section{LXXXVI}

„Höhe“ passen nicht hierher. Vielleicht darf man an eine Ableitung von gordatzen denken, welches auch "beschützen“ bedeutet, aber unbedenklich ist das keineswegs. Schmiegte sich die Uebersetzung knapp an die andern Texte an, so würden wir wahrscheinlich den Schlüssel für das Wort in der Hand haben. Freilich ist mir gu bay 408\%17 dem Sinne nach ganz klar, es ist so viel wie $=g u$ beçala; aber so kenne ich es sonst nicht (vgl. hiri bay becembat $\left.38^{\mathrm{r}} 14\right)$.

Wie bei den Buchstaben, lasse ich auch bei den Wörtern auf die qualitativen die quantitativen Varianten und Fehler folgen. Was als Pleonasmus und als Ellipse zu bezeichnen ist, ist nicht immer leicht festzustellen; wir können oft die Norm ebensowohl auf die eine wie auf die andere Seite legen. Das gilt hauptsächlich für den Gebrauch des (bestimmten) Artikels. Neben dem Possessivpronomen wird derselbe bei L. bald gesetzt, bald nicht, z. B.:

bere anayea $423^{r} 12$.

bere anayeari $425^{\mathrm{r}} 20$.

neure buruäz $169^{\prime} 31$.

gure Iaincoaren $444^{\prime} 10$.

bere anaye $426^{\circ} 16$.

bere anayeri $423^{\mathrm{r}} 15$.

neure buruz $169^{1} 30$.

gure Iaincoren $444^{\prime} 10$.

Ich habe den kaum übersehbaren Stoff auf die Häufigkeitsverhältnisse hier nicht näher untersucht. Das Prädikat pflegt, wenn es Partizip (ausserhalb der zusammengesetzten Tempus) ist, den Artikel zu haben, wenn Substantiv und Adjektiv, nicht; sehr auffällig zeigt sich das z. B. in : egoiten baçarete . . . . fundatuac eta fermu $354^{\prime} 23$. Ausnahmen kommen vor; vgl. z. B.:

hayen obrúc gaichtoac bai- haren obrác gaichto diradela tirade $164^{1} 19$. $174^{1} 7$.

Wie es beim Possessivpronomen ist, weiss ich nicht; es findet sich Beides, so:

hirea duc refumá $9^{1} 13 . \quad$ gure içanen da heretagea $85^{1} 7$. In einer Reihe von Fällen bin ich geneigt das Fehlen des Artikels als einen wirklichen Druckfehler zu betrachten, so in:

Iefus Chriften fedez $270^{1} 22$. Iefius Chriften fedeaz $333^{\circ} 16$. $335^{r} 22$.

gaineracoz $292^{r} 16$.

Gaineracoaz $378^{\prime} 8 . \quad 430^{-24}$. $\mathrm{G} \mathrm{v}^{\mathrm{r}} 1$.

haragui guciren gainera haraguiguciaren gainean 196'2. $209 \times 17$.

Iaincoren templean $40^{\mathrm{r}} 12$. Iaincoaren templean $365^{\mathrm{I}} 4$

Iainco baithan $425^{\mathrm{r}} 16$. u. s. w.

laincoa baithan $424^{\prime} 15$ u. s. w. Und ebenso in andern Fällen das Stehen des Artikels, so in vicitzearen $169^{1} 26$, da vicitze vorausgeht und der griechische 


\section{LXXXVII}

wie der französische Text den Artikel nicht haben. Schwankungen wie der Artikel, unterliegen auch andere Wörtchen; vgl. z. B.:

ceren .... hautfi suen ceren.... erran baitzuen ebd. $169 \cdot 18$.

badaquiçue vdá hurbil dela badaquigue ecen vdá hurbil dela $48^{\mathrm{r}} 32$. 89 .28.

Zuweilen bleibt bei L. ein Wort des Urtextes ohne Entsprechung, und zwar auch so dass im baskischen Text selbst eine Lücke, formaler oder sachlicher Art, fühlbar wird:

vicitze [emaiten] draucana $172 ' 33$.

emazte [Iudu] fidel 238'1.

[eta] Iefus $248^{\prime} 24$.

Hierzu füge man:

[Areopagus] Marfen cofk $459^{1} \mathrm{a} 2 \cdot 2$.

gaua [9] Kal. vi'1.

In afto eme vztarrico batec $418^{\circ} 16$ ist das zweite Wort ron úro-

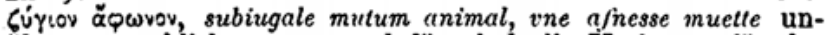
übersetzt geblieben (mutu); dafür sind die Varianten für das erste nebeneinander wiedergegeben worden. - Dass ein Wort wirklich zu viel ist, kommt begreiflicherweise seltener vor. Die sinnlose Wiederholung eines Wortes wie wir sie in: erruniçã içũ 7 '21

wahruehmen (wo in den verschiedenen Exemplaren das eine içã gestrichen ist), stellt einen Druckfehler dar wie er überhaupt nicht oft begegnet. [Ich schalte hier die Erwähnung ein dass die drei letzten Worte von 183'26, welche ihr Entsprechendes in der Vulgata nicht haben, im Leipziger Exemplar gestrichen sind, also wohl von der Hand eines katholischen Basken; die Tinte ist aber eine andere, dunklere als die mit welcher auf dem Titelblatt unter dem Wappen geschrieben steht $\grave{i}$ L'vjage de fr. franşois de Toloze und über demselben Huguenot, was wiederum mit dunklerer Tinte getilgt ist.] Ein Worttheil ist um eine ganze Zeile verschoben worden:

tzea eta bere . . . . . . für: tretenitzea eta bere. . . . . . . treteni creatzea $\left(\mathrm{Cr}^{\mathrm{i}} 25 \mathrm{f}\right.$.) creatzea?

Endlich ist noch zu erwähnen dass das Baskische eine gewiese Freiheit der Wortstellung kennt*), dass also bei $L$. Varianten auch dieser Art bestehen, z. B.:

*) Ich benutze diese Gelegenheit um auf die Wichtigkeit hin. zuweisen welche die Wortstellung in den des Hülfsverbs ermangeln- 


\section{LXXXVIII}

ez nekatzen dirade, ez iruten eztirade nekatzen, eta eztute duté $10^{1} 28$. irutẽ $130^{\mathrm{r}} 27$,

dass er aber in ein paar Fällen diese Freiheit überschritten zu haben scheint, so in Iaincoaganic magiftru ethorria $163^{1} 2$ für m. I. e., in landaco egun belhar dena $10^{1} 30$ für b. e. l. d. und in der entsprechenden Stelle egun landán belhar dena $130 \times 28$ für b. e. $l$. d. oder auch e. l. den belharra.

Druckversehen welche ganze Sätze zum Gegenstand haben, kommen überhaupt nur ausnahmsweise vor; am Ehesten noch wird ein Satz ausgelassen. Bei L. ist mir Nichts dergleichen aufgestossen. Zu dem Stern $295^{\circ} 1$ fehlt die Angabe der Parallelstelle; die franz. Uebersetzung hat zwar hier keine (auch keinen Stern), wohl aber die Vulgata.

Ausser den schon oben S. XVII zusammengestellten Zahlendruckfehlern, die sich auf die Seiten beziehen, kommen verschiedene am Anfange der einzelnen Verse vor:

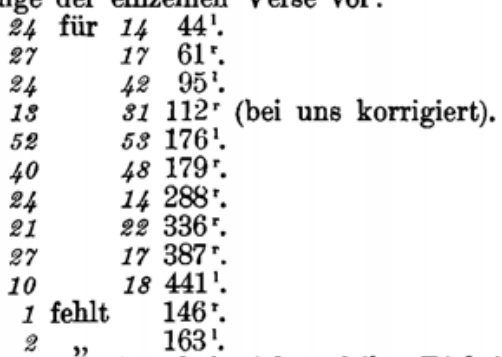

Die Verszahlen der Ueberschriften habe ich auf ihre Richtig-

den Sätzen der Ueberschriften und des Sachverzeichnisses für die Bestätigung des passiven Charakters des transitiven Verbs und des instrumentalen Charakters des Aktivus besitzt; vgl. z. B. Bi Aingueru Mariac ikuften $203^{\text {× }}$ Ü. „Zwei Engel von Maria im Gesehenwerden", Diacreac ordenatzen $216^{1} \dot{U}$. "Les diacres ordonnez", bi teflimonio beftiác hil $442^{1}$ Ü. ,zwei Zeugen von den Thieren ge-

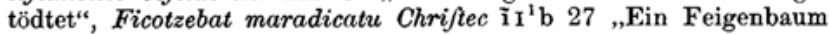
von Christus verflucht". Ein Beweisstück andrer Art für die gleiche Thatsache ist das bäufige Iaincoac manu beçala (z. B. B III $^{1} 30$. vi $\left.{ }^{1} 30\right)$,gemäss dem Befehl von Gott", wobei allerdings nicht zu überschen ist dass manu (span. mando) in andern Formeln geradezu in der Rolle eines Partizips zu mandatzen erscheint, so hic manu drauzquiguanac Abc A vII ${ }^{\mathrm{r}} 24$ (vgl. das unmittelbar vorhergehende hic debetu drauzquiguan; debetu ist \} span. deviedo). 
keit hin nicht untersucht; jedenfalls finden sich darunter solche die schon der Reihenfolge nach falsch sein müssen, so:

$\begin{array}{llll}27 & \text { für } & 25 & 223^{1} \\ 18 & 28 & 276^{1} \\ 19 & 10 & 414^{\circ} .\end{array}$

Ebensowenig habe ich mich auf die Zahlen der Parallelstellen eingelassen. Bei den Signaturen fehlt oder steht $i$. manchmal gegen das Prinzip der ganzen Reihe, so $i .,(k$. $i .)$,$l ., (m. i.,) n.,$ $(o . i .$,$) p., (q . i .$,$) r., in den Oth. eccl. (E.,) F . i .,(G)$.

Die Hülfszeichen lassen sich bequem in drei Klassen zerlegen: phonetische (Trems, Akzent; von den Abkürzungszeichen, insbesondere Cedille und 'Iilde wird hier abgesehen), morphologische (Binde- und Theilungsstrich), syntaktische (Interpunkzionszeichen: Beistrich, Punkt, Doppelpunkt, Fragezeichen, Ausrufungszeichen). Sie geben reichen Anlass zu Bemerkungen über folgewidrigen oder fehlerhaften Gebrauch.

Das Trema bei L. schliesst sich auch in seiner Anwendung an das französische jener Zeit (veuë, fouët, aduouëra) an, wenigstens im Allgemeinen. Es wird nämlich auf einen Vokal, und zwar auf $a$ oder $e$, nur nach $u$ gesetzt, um auszudrücken dass dieses $\mathrm{zu}$ einer andern Silbe gehört, entweder den einzigen Vokal derselben bildet oder den zweiten Theil eines Diphthongen. Aber beide Fälle sind wiederum beschränkt. Der erstere auf ein dem $u$ vorausgehendes $g$ (damit man nicht an ein stummes oder halbvokalisches $u$ denke) und einfaches $r$ : mainguäc, malguëc $\left(297^{1} 10\right)$, burıä, cervä, buruën, ceruëtan. u. s. w.; hingegen difcipulua, cerbitzua, defertua, bekatua, jaindua, difcipuluey u. 8. w. Doch nach beiden Seiten hin fehlt es nicht an Ausnahmen; so finden wir einerseits z. B. liburuan $86^{1} 26$, liburua $455^{1} 12$, desperiuruacgatic $368^{\circ} 10$, anderseits z. B. daritzuëney $8^{1} 44$ (daritzueney $8^{1} 46$ ), deabruäc $14^{\mathrm{r}} 31$. Im zweiten Falle handelt es sich um $a$ und $e$ nur nach au. L. schreibt gauä, gauären und daneben auch gaua, gauaren, aber regelmä8sig gauaz; auch sonst kommt auä vor, so nauäla $53^{3} 34$. Auë dürfte vor $a$ das Herrschende sein: drauëan, drauëagu, nauëanagatic u. s. w., doch auch diraueat $\left(33^{\circ} 16.197^{5} 6\right.$. 14). Vor Konsonanten und am Wortende dünkt mich aue ohne Trema häufiger zu sein als mit Trema; doch z. B. arauëzco $373^{r} 3$, dauëney $38^{1} 23$, erauën $50^{r} 27$, hauëc ${ }^{* *} \mathrm{III}^{r} 15$, baitrauë $\widetilde{a} \mathrm{II}^{1} \mathrm{~b} 6$, ezpalitzaue ${ }^{*}$ VIII ${ }^{1} 36$. Ganz ausnahmsweise steht das Trema nach o: Circoncifionecoëtarat $333^{\circ} 9$. Ein paar Mal ist $i$ mit dem im Baskischen unverwendbaren $i$ verwechselt worden: pïer $381^{\circ} 9 \mathrm{R}$. (nicht wiedergegeben; der zweite Punkt ist sehr schwach), -gatïc ã IV ${ }^{\prime} \mathrm{b} 24$, $\ddot{i}$ çan $\mathrm{a}^{\prime} \mathrm{v}^{1}$ a 24 (der Doppelpunkt in 
ircater i vi ${ }^{r} \mathrm{~b} 35$ zeigt sich bei scharfer Betrachtung im St. als ein in zwei Theile auseinanderfallendes $\sim$; im $L$. als der Schimmer eines solchen).

Dass der Akzent, und zwar ist es der Akut, bei L. den stärkst betonten Vokal des Wortes bezeichnen soll, darüber kann kaum ein Zweifel bestehen. L. hat ihn zu diesem Zwecke eingeführt - wenigstens Dechepare kennt ihn vicht - und einen umfassenden Gebrauch von ihm gemacht. Die Akzente L.s bilden somit einen werthvollen Beitrag zu dem wichtigsten, aber bisher ganz vernachlässigten Theil der baskischen Sprachstudien, dem der sich auf die Betonung bezieht. Ich enthalte mich hier mit besonderer Strenge aller Vergleichungen und Schlussfolgerungen; ich bemühe mich nur zu erkennen welche Regeln L. befolgen wollte. Es ist dies deshalb nicht leicht weil er sie jedesfalls nicht mit genügender Sorgfalt befolgt hat; es ereignet sich dass unter ganz gleichen Bedingungen der Akzent ebenso oft fehlt wie vorhanden ist. Wir werden den vorhandenen Zeichen eine grössere Beweiskraft beimessen als den fehlenden; nur wenn die positiven Fälle zu sehr in der Minderheit sind, werden wir geneigt sein sie auf Rechnung des Irrthums zu setzen. Die folgende Zusammenstellung bitte ich nicht anders denn als einen vorläufigen Versuch zu betrachten.

1) Der Akzent steht meistens auf der letzten Silbe, und zwar:

a) auf einem aus zwei zusammengezogenen Vokal:

a) in der Deklinazion:

a) auf dem $a\} a$ des Stammauslautes $+a$ des Artikels. So alculsa "Tochter", alabá ,die Tochter"; alabaz „durch Tochter", alabáz "durch die Tochter"; alabac "von Tochter", alabác "von der Tochter" und ,die Töchter". "Es dient also der Akzent dazu die bestimmten Formen von den unbestimmten zu scheiden, vgl. z. B. Eliçá ezten Eliça G v1'15 „dass die Kirche nicht Kirche sei“ (doch eignet er auch dem Lokativ: gloriún, obwohl der unbestimmte gloriatan lautet); innerhalb jener fallen der Akt. Sing. und der Nom. Plur. zusammen. Unbeabsichtigt ist die damit verbundene Scheidung der auf $a$ ausgehenden Nomen von den nicht auf $a$ ausgehenden innerhalb der bestimmten Deklinazion (guiçon "Mensch“, guiçona „der Mensch", guiçonaz ,durch den Menschen", guiçonac "von dem Menschen" und "die Menschen"), aber sie ist für uns Fremde oft sehr nützlich; denn da das baskische Substantiv nur selten im artikellosen Nominativ vorkommt, so entstehen leicht Zweifel über seine Gestalt. Herdoil z. B. gibt van Eys als 
Wort für "Rost" an; aber bei $\mathrm{L}$. weniggtens lautet es herdoilla (entsprechend dem gask. arroudilho), wie aus herdoillác $10^{r} 19$ neben cerrenac hervorgeht, deutlicher allerdings noch aus dem herdoillac des folgenden Verses neben cerrenec. Der Akzent fehlt nicht selten da wo er stehen sollte, z. B. hiraca $24^{1} 25$. 25r26. 27 = hiracá $25^{\prime} 30$. ' 38 . 40, eguia 179'45 = eguiá 179 '46, und steht nicht selten wo er fehlen sollte, z. B. iguiteyá $67^{1} 29=$ iguileya $447^{\prime} 15.448^{\prime} 16$ (iguitey $447^{\prime} 14 .^{\prime} 448^{\prime} 17.18 .19$ ), principalá B VIII $^{r}$ 9. Das Erstere ist natürlich häufiger; so findet sich z. B. unter 21 Fällen copá und copa je 7 Mal richtig, $6 \mathrm{Mal}$

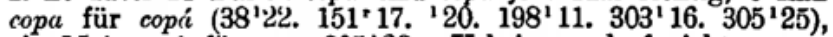
ein Mal copá für copa $305^{1} 28$. Uebrigens darf nicht ausser Acht gelassen werden dass ähnlich wie im Romanischen und in andern Sprachgruppen, der Artikel zuweilen mit dem Substantiv verwächst und umgekehrt wiederum das stammhafte $-a$ als Artikel behandelt wird. Van Eys führt in seinem Wtb. Substantive auf -go an, wie bekaizgo, erhogo, etsaigo, und zitiert dafür auch Leiçarraga. Aber bei diesem finden sich diese und die entsprechenden Substantive nur mit der Endung -goa, wie sich sowohl unmittelbar aus den artikellosen Formen (80 292'18. 23. $293^{1} 14.294^{1} 19 .{ }^{*} v^{\prime} 14 .{ }^{* *} v^{1} 22$ u. 8. w.) als aus dem Akzent der mit dem Artikel rersehenen ergibt. Und so werden sie, für die franz.-bask. Mdd., überall da verzeichnet wo der Artikel nicht hinzugefügt wird; ausdrücklich wird -goa als die Endung angegeben z. B. bei d'Abbadie und Chaho (BB 216) S. 21 mit den Beispielen: handigoa, chipigoa, edergoa, mouthilgoa, und bei Salaberry S. 188: ,goa, titre ou qualité; Aita saindurgoa, papauté.... erior-goa, cure.....nausi-goa qualité de maître." Dennoch glaube ich dass die ursprüngliche Endung -go ist (Manterola [BB 495] schreibt im Wtb. etsaigo-a); nur unterliegt die von van Eys und Manterola angenommene Gleichheit derselben mit der Adjektivendung - $c o$ gewissen lautlichen und begrifflichen Bedenken. In manchen Wörtern erscheint mundartlich - ๓sa für -goa, so in beknizcoa; und auch L. hat ahacoa $218^{\prime} 14$ (vgl. ahaide und zu diesem wiederum aurhide), welches auch bei Larramendi gebucht ist. Ich vermuthe dass -go(a) zunächst zur Bezeichnung einer Würde gedient hat, entsprechend dem altspan. -azgo, so hat L. embachadoregoa $\left({ }^{* *} \mathrm{III} 20\right)$, emperadoregoa $\left(\widetilde{\mathrm{x}^{\prime}} \mathrm{v}^{\prime} \mathrm{b} 34\right)$, iaurgoa $\left(\mathrm{B} \mathrm{v}^{\prime} 2\right.$. $\mathrm{CVI}^{\prime} 32$; von iaun, wie auch iauregui mit $r$ fïr $n$, vgl. belhauric- S.XXXIX), mandatalgoa $\left({ }^{* *} \mathrm{IV}^{1} 7\right)$, facrificadoregoa $\left(410^{1} 5\right.$. бIII' $a$ 39); Bildungen die auf Adjektive zurückgehen, wie aufurtgoa $\left({ }^{*} \mathrm{~V}^{\prime} 14\right)$, gogorgoa (** ${ }^{*} \mathrm{VII}^{\prime} 17$. EvII '9), ielofgoa $\left(326^{1} 2\right)$ scheinen mir jünger zu sein. Wie leicht. der Artikel sich 


\section{XCII}

einstiehlt, bezeugt algoaric $\mathrm{B}$ vir ${ }^{1} 35$, in dem ich nichts Anderes als das span. algo "Etwas" wiederfinden kann, das also für *algoric steht.

bb) auf dem $e$ der pluralischen Kasus, indem dieses

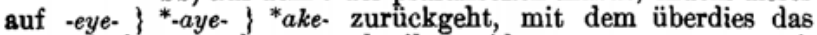
stammhafte $-e$ und $-a$ verschmilzt. Also von guiçon: guiçonéc „von den Menschen“, guiçonén „der Menschen“, guiçonéz „durch die Menschen"; von emazte: emaztéc ,von den Frauen" u. в. w. Hier scheidet der Akzent von den unbestimmten Formen : guiçonec "von Menschen" (ab homine), emaztec "von Frau". Ebenso wird alabéc, alabén, alabéz geschrieben, obwohl hier keine Verwechselung möglich ist. Ảber der Dativ Plur. guisoney, emaztey, alabey trägt meistens keinen Akzent auf der Endung (z. B. Bl. 7-1619 Mal -ey, $2 \mathrm{Mal}$-éy), da diese überhaupt kein unbetontes Gegenstück hat; dass er immerhin nicht selten gesetzt wird, daran ist die Analogie mit den andern Kasus schuld. Man bemerke: çuhurréy eta adituey $20^{\circ} 25$. 124 21 . Dass gelegentlich auch in den andern Fällen der Akzent weggeblieben ist, bedarf keiner weiteren Belege. Das -én hat sich auch in derjenigen Form festgesetzt durch deren Zusammenziehung es erst entstanden ist: hayén „illorum" für *ha-k-en; ebenso horién für "kori-k-en ,istorum", hauén „horum" für *hau-k-en. Auch dieses hat Zusammenziehung erfahren: -ón, z. B. in ceurón $31^{\circ} 8$ (ceuron $88^{\circ}$ ), gución 11'32 (gucion 350.17), dazu -óc\} hauec, z. B. in ceuróc $72^{r} 31.80^{\circ} 50$, ducuenóc 12r23; der Akzent fehlt z. B. in itfuoc 16r28, gucioc ${ }^{* *} \mathrm{I}^{\mathrm{r}} 34$, bes. in den entsprechenden Dativen, so duçuenoy $67^{\circ} 24$, abratfoy $111^{1} 24$, çaretenoy $111^{1} 25$, çaudetenoy $112^{\mathrm{r}} 25$, dançusuenoy $112^{\mathrm{r}} 27$. Die unbestimmte Deklinazion des Relativpronomens galt ursprünglich auch für die Bezeichnung des Plurals; L. hat noch cein auch als Nom. Plur. (z. B. $241^{\prime} 10$. $365^{\circ} 5$. 9), dann sind wohl ceinéc, ceinén, ceinéz (ceinéz E VII ${ }^{1} 12$ dürfte singularisch sein) als pluralische Formen, da es nur einen bestimmten Plural gibt, aus den unbestimmten ceinec, ceinen, ceinez differenziert (andere Mundarten kennen allerdings, wohl nach romanischem Muster, auch einen bestimmten Singular: ceina, ceinac u. s. w., und L. hat wenigstens ceinagatic), und ihnen hat sich ceiney gegenüber dem ceini des Sing. angeschlossen. Bién, himurén $131^{1} 52$ zu den ebenda belegten Nominativen biac, hirurac sind ganz in der Ordnung; aber warum biguec $34^{1} 19$. $48^{\circ} 41$, nicht biguéc zum Nom. biga $=$ biac?

$\beta$ ) in der Konjugazion, und zwar auf dem -a- ? *aka-, in welchem zunächst $k$ als Zeichen der 2. P. S. des Urhebers enthalten ist; so z. B. deşán $11^{1} 5$, ditzán $69^{\circ} 23$, dieģadán $72^{*} 25$, 


\section{XCIII}

drauztán $197^{\circ}$ 6. Diese Formen sind nur durch den Akzent von andern geschieden, die die 3. P. S. als Urheber haben (descin „dass du ihn habest", deçan „dass er ihn habe"); daher fehlt bei ihnen der Akzent kaum. "Sodann kann das in dem $-\dot{a}$ - enthaltene $-k$ - auch den eigentlichen oder den ethischen Dativ darstellen, z. B. dieçán E $\mathrm{vI}^{\prime} 10$, cieçán $147^{\prime} 31$, guiniabiltzán $103^{\circ} 48$, welchen keine Formen ohne Akzent gegenüberstehen. - Ich habe diese Fälle aus rein äusserlicher Rücksicht hier eingeordnet; es ist mir sehr wahrscheinlich dass sie mit den unter c) $\alpha$ ) zu besprechenden ursächlich zusammenhängen, mit andern Worten dass die Zusarnmenziehung von -aa- * $-a k a-^{*}$ für die Akzentsetzung bedeutungslos ist, indem L. auch wenn er beide Vokale nebeneinander geschrieben hätte (wie das auderswo wirkiich vorkommt) den Akzent nicht gespart haben würde: -aá-.

b) im Anruf. Der Akzent bildet wiederum Scheideformen : a) im Vokativ. So zunächst bei Eigennamen, wie $\mathrm{Abra}$ hám 139r24. ${ }^{1} 30$, Mariá $203^{1} 16$, Philippé $192^{\circ} 9$, Simón $91^{\prime} 37$. Doch keineswegs immer; vgl. z. B. Martha, Martha $125^{1} 41$ und sogar häufiger Simon $33^{1} 25$. $^{1} 1^{1} 40$. (doppelt) $152^{\mathrm{r}} 31$. Ferner bei artikellosen Gattungsnamen, so Aitá 20'25. ${ }^{12} 26$ u. s. w., Semé $41^{\prime} 28.62^{1} 5$ u. s. w. (doch auch jemé wo es nicht Vokativ ist: $98^{\prime} 32.148^{\prime} 44.335^{\prime} 7$ ), anayé $112^{\prime} 42.224^{\prime} 17$. $383^{\prime} 7$, o Regué 260'13. Bei solchen mit Artikel, so Magi/truá 52' 25 . $53^{\prime} 49$ u. s. w., o guiçoná $268^{\mathrm{r}} 1$, Ene haourrá $399^{\mathrm{r}} 5$, deabruaren femeá $232^{\prime} 10$, Dauid-en Jemeá $143^{1} 38$ (Dauid-en Jemea $143^{1} 39$ ). Endlich bei Pluralen, so anayeác $209^{\prime} 29.210^{r} 37$ u. s. w., HAourrác $346^{*} 1$, Iuduác, guciác 209¹4, guiçonác 209²2, Emazteác, Senharrác, Haourrác, Cerbitzariác $357^{1} 18 \mathrm{ff}$.

ß) im imperativischen Ortsadverb. So huná „siehe hier": huna "hierher", horrá ,siehe da": horra "dahin", hará „siehe dort": hara „dorthin". Man findet z. B. huná und huna in beiden Bedeutungen nebeneinander $14^{r} 29.435^{\prime} 1$. Zuweilen fehlt der Akzent wo er stehen sollte, so horra $20^{\circ} 19$, huna $23^{\circ} 41.128^{\circ} 41$ u. s. w., huna hemen, hara han $140^{1} 23$ (huná hemen, hará han $\left.140^{1} 21\right)$. Seltener findet sich das Umgekehrte, so huná $443^{\prime} 12$.

ү) im Imperativ. Hier vermuthe ich nur dass z. B. ohí "gehe" und oha "du gehst" unterschieden werden sollen. Das Letztere kommt selten vor $\left(195^{r} 5\right)$, das Erstere ungemein häufig und wird dann meistens ebenso geschrieben $\left(13^{\mathrm{r}} 9.34^{1} 15 \mathrm{u}\right.$. s. w.); doch ohá $13^{1} 13.62^{*} 44.109^{\circ} 14$. So wohl auch aicén, sei" $3^{r} 13$. 7'25. $13^{r} 3$. $62^{\circ} 41$. $109^{\circ} 13$. A vir 27 . VII 2 . Div' 30 zum Unterschied von aicen ,der du bist" oder „du seiest". 


\section{XCIV}

Auch eznaicén 328 29 (ebenda und ebenso eznaicen) ist imperativisch (,,ich sollte nicht sein $\left.?^{\prime \prime}\right)$, naicén $175^{1} 28$. $308^{\prime} 12$ allerdings nicht. Vgl. ezgarén DVII ${ }^{1} 26$, garén $\mathrm{EnI}^{\mathrm{r}} 13$ und rein imperativisch GOacén Cvi' 7 .

c) auf pronominalen Elementen der Verbalformen :

a) auf -(k)a- und -na- der 2.P.S. als Urheber oder Ziel (die Bezugsformen inbegriffen), z. B. duán $13^{\prime} 13$, cituán $86^{\prime} 20$, nián $50^{\circ} 24$, diát $50^{\circ} 25$, drauzquiát $20^{\circ} 25$, drauzquioán $196^{1} 2$, ezquindrauqueán $199^{1} 30$, çateán $20^{\circ} 23$, çauán $112^{*} 30$, ciedián $43^{\circ} 27$, eztioffát $35^{\circ} \cdot 22$, baniaquián $186^{\circ} 42$; dunán $29^{1} 28$, Eztinát $93^{r} 68$, dionán $55^{r} 70.93^{r} 68$. Sehr häufig fehlt der Akzent, z. B. diat $60^{\mathrm{r}} 2.378^{1} 11.12 .379^{1} 20.381^{1} 8$, citiat $36^{1} 20$, draut $31^{\circ} 18$, dirauzteat $197 \times 8$, dioffat $63^{\circ} 11$; draunat $71^{1} 22.23$, Etzeaquinat $55^{\circ} 70.93^{\circ} 68$; vielleicht in gewissen Formen regelmässig, wie denen mit -auea-, so diraueat $33^{r} 16$, dirauëagu $126^{r} 4$.

B) auf dem $-o(-a)$ und dem $-e \operatorname{der} 3$. P. S. und Pl. als Ziel, z. B. çayó $3^{\mathrm{r}} 13$, cequión $3^{1} 19$, badaquió $11^{i} 9$, cieçón $4^{1} 15$, ieģoc $8^{1} 39$, emóc $8^{1} 42$, diotfó $5^{1} 6$, diotjă $92^{\circ} 45$, baderró $74^{r} 11$, daritzón $346^{\circ} 33$, daritzí $425^{\prime} 1$; cequién $30^{\prime} 5$, cieçén $3{ }^{\circ} 8$, iecéc $38^{\circ} 8$, lequiztén $108^{1} 7$, çaquiztéz $401^{\circ} 17$, darizté $44^{\prime} 6$. Zahlreich sind die Fälle in denen man den Akzent vermisst, z. B. cieşon $5^{1} 7$, ieşoc $8^{1} 40$, ciecen $15^{1} 15$, cequien $59^{r} 9$, çaquiztez $216^{1} 38$.

Y) auf dem te $(-e)$ der 3. P. Pl. als Urheber, z. B. dieçaquedé * vI ${ }^{\mathrm{r}} 27$, duté $2^{1} 23$, dié $33^{\mathrm{r}} 16$, nauté $59^{\circ} 10$, dituzté $7 \mathrm{r} 12$, citié $13^{1} 20$, gutén $30^{1} 7$, lutén $\mathrm{F} \mathrm{VII}^{1} 9$, ceguitén $92^{\mathrm{r}} 50$, cioé 434' 9 ; dagegen auch z. B. baitute $6^{\prime} 5.14^{\text {r }} 27.25^{\prime} 40$, ezpaitute $15^{1} 14$, dituzte $24^{\circ}$ 7. $25^{1} 41$. 42. Es kommt der Akzent auch auf dem te als Pluralzeichen für das ç- der 2. P. vor: etzinaquitén $103^{\prime} 49$, bacindarizté $193^{\prime} 28$, caituztéz $353^{\circ} 2^{\prime} 2.358^{\prime} 9.10$, daneben freilich çaituztez $314^{1} 19.20$. $^{1} 330^{\prime} 12.353^{r} 21.358^{1} 14$, baçaituztez $327^{\prime} 20$. Nur ausnahmsweise wohl auf dem -de der 3. P. Pl. als Subjekt bei Intransitiven: ciradén 164'23 (neben ciraden).

d) in Postposizionen :

a) auf -at in den Verbindungen -rát, -şát oder -tzít, -ganát, wie Babylonerát $1^{1} 12$, harát $46^{\circ} 39$; arimẽcát $20^{1} 29$, dençát $13^{\circ} 4$, Dathorrençát $46^{\mp} 35$, bidecotzát $17^{\times} 10$, doctrinatzát $28^{\prime} 9$; Aitaganát $195^{1} 16$. Die Formen ohne Akzent sind sehr häufig, z. B. Babylonerat $1^{\prime} 11$, harat $54^{1} 64$; dèçat $9^{5} 4$, erratzecotzat $25 \times 30$; harenganat $81 \div 21$.

B) auf dem distributiven -na, -ra, so baná $38^{\mathrm{r}} 10$, hirurná $162^{\prime} 6$, hamarná $72^{\prime} 40$, ehuná ebd., birá $17^{\prime} 10$.

e) in gewissen Formen von içan. Zunächst im Imperfekt, 
welches das einzige ist wo der Stammvokal dem - $n$ unmittelbar vorhergeht, z. B. nincén $50^{1} 36.43$, incén $54^{1} 69$, guenén $264^{\prime} 14$, und natürlich auch bacén $\widetilde{a} \mathrm{IV}^{1} \mathrm{~b} 26.27$, baitzén $67^{1} 37$, etzén $84^{\circ} 13$, licén $89^{\circ} 34$, baguinén $263^{\prime} 37$, baiquenén $262^{\prime} 18$, ezquinén 262' 21 . Es kommen aber auch Formen ohne Akzent vor, so guinen $335^{\mathrm{r}} 23$. Auf cegoén $70^{1} 6$, nenguién $328^{\mathrm{r}} 33$ hat er kaum etwas zu bedeuten. Nicht anders aber wie mit etzén wird es sich mit dem entsprechenden Präsens eztén verhalten, welches allerdings vielfach imperativisch oder konjunktivisch ist (so $47^{\circ}$ 20. $337^{r} 13.412^{\prime} 3$. $^{* *} \mathrm{vI}^{\prime} 39, \mathrm{D}^{2} \mathrm{vII}^{1} 3$ ), sodass es dann unter b) $\gamma$ ) Platz finden könnte, aber doch wohl nicht minder oft relativ (Bo ẽ $\mathrm{VI}^{r} \mathrm{~b} 30$. BII 36 . VII ${ }^{1} 14$. CVI ${ }^{\mathrm{r}} 21$. F vII' 3$)$.

f) Vereinzeltes, Zweifelhaftes, Irrthümliches. Herén wird meistens so geschrieben, z. B. $440^{\circ} 11.12$ vier Mal, etwas seltener heren $58^{\prime} 64$; die andern Ordnungszahlen lehen, bigarren u. 8. w. sind nicht akzentuiert (doch Lehén ã vIII ${ }^{r} 3$ ); sollte dort $e$ aus zwei Vokalen zusammengezogen worden sein (von der Grundzahl hirur)? Wie es mit afjereré $42^{r} 7$ (af/erre $3^{1} 16$. $344^{r} 26$. $445^{\mathrm{r}} 17$ ), beránt $225^{1} 38$, bethé $193^{1} 11$, ezquér $249^{1} 3$, gabéz $73^{1} 2$ u. a. steht, darüber erlaubt das beschränkte Vorkommen und der Mangel an Analogieen kein Urtheil. - L. hatte gewiss nicht die feste Absicht die fast durchgängige Endbetonung der mit einem Konsonanten schliessenden Fremdwörter zu bezeichnen, er hat sich nur hie und da dazu verleiten lassen, so conjeillér $95^{\prime} 43$ (onfeiller $1^{5} 6^{\prime} 50$ ), prejónér $5^{\prime} 12.257^{\prime} 27.342^{\prime} 1$ (prejoner $56^{\circ}$ 16. $343^{r} 1$ ), eftrangér $341^{1} 19.397^{r} 13$ (eftranger $\left.341^{\circ} 12\right)$, defpenjér 131'42. 138'2, paftór 400' Ü. (Pajtor 343'11. 414'4), fuccejJór 256'U. (fuccejJor 257'27), differént Cv'39R., obediént $\mathrm{DVI}^{\mathrm{r}} 16$, burgés $136^{1} 15$, tornés ã $\mathrm{II}^{1} \mathrm{~b} 19$. Sollen wir nun etwa auch in gendarmés $255^{r} 23.376^{\circ} 3$. व̃ III $^{r} \mathrm{~b} 30$ eine Wiederspiegelung der baskischen Aussprache sehen? 226 7 steht honmedarmes. - Als Versehen sind aber gewiss oder mit grösster Wahrscheinlichkeit zu betrachten Akzentsetzungen wie behá D vII ${ }^{r} 15$. EIV $^{r} 20$ (neben beha ebend. 21), beré $33^{1} 25$, biribilgaturén $384^{\prime} 12$, erranén (du) $404^{\prime} 18$, Core-erén $429^{\circ} 11$, harén $\mathrm{E}^{1}{ }^{14}$. 26, munduarén $47^{\mathrm{r}} 21$, repoftarén $\mathrm{FIV}^{1} 32$, bozcarióz $26^{\circ} 44$ (das Akzentzeichen ist schwach und unregelmässig), contrá $337^{\prime} 17$ (in demselben Vers zwei Mal contra), delá Crv'21. G ${ }^{\prime} 30$, denáz $\mathrm{C} \mathrm{vi}^{{ }^{2} 27}$ (denaz ebend. 28), harçáz CvII'6, deteftutzén Giv'25, eguitén (du) $\mathrm{DrV}^{\mathrm{r}} 19$, ethortén $\mathrm{GV}^{\mathrm{r}} 5$, Thardefién (diát) $256^{1} 10$, diradelaric $\mathrm{GvI}^{1} 13$, hartaric 14²8, Ecén 333r12. Eir'33, emán Cvir31. Fvir24 R., eracutfaçué 324'24, erorcór A III' 39, guiçón 92' 51 , hurú $57^{\prime} 36$, libré 


\section{XCVI}

$275^{\circ} 20$, neceffarió $125^{\prime} 42$, oráin $290^{\prime} 26$, vkán $366^{\prime} 10$. Andere sind schon früher erwāhnt worden. Wenn eguitecotán $\mathrm{DI}^{1} 35$, errotán $48^{\circ} 41$, hunetán * ${ }^{*}{ }^{1} 30$ (gleich darunter hunetan), halatán CIII'16. V'22. G II'13, haourretán FII'19 R., itzultzecotán $144^{1} 12$ durch nolatán $90^{\prime} 11.98^{\prime} 18.309^{\prime} 16 . \mathrm{Em}^{\prime} 30$ gestützt zu werden scheinen, so steht diesem doch ein ausserordentlich häufiges nolatan entgegen: $21^{\prime} 14$. $22^{\prime} 26$. $^{1} 34.40^{\prime} 20$. 54'54. 65'23. $66^{1} 13$ u. 8. w. In gucietán $321^{\circ} 4$ hat natürlich das Zeichen den Platz verfehlt (guciétan). - In báy 293'10 und éz 323'10 steckt vielleicht die Bezeichnung des Satztones. Ebenso wird dén $A v^{\prime} 9$ als (erran)dén zu deuten sein wie ja Andere in einem Worte schreiben. Man bemerke noch céin $\mathrm{BI}^{\prime} 30$.

2) Der Akzent steht ausnahmsweise auf der vorletzten Silbe und zwar soweit sich mit Sicherheit erkennen lässt, im Anschluss an die Setzung auf der letzten Silbe; doch nur in zwei Hauptfällen:

a) auf dem -a. \}-aka- und -ka- (s. oben 1) c) a)), vor Allem vor -la, z. B. duála $42^{\prime} 16$, dituála $34^{\prime} 8$ (dituala $34{ }^{\prime} 9$ ), ezteçála $8^{1} 42$, ezgaitzála $9^{\prime} 13$, dacuffála $55^{\prime} 4$. Vor dem Artikel, so duána $312^{\prime} 36$; doch gayana $224^{\prime} 17$, drauzquidanac 197 9 . 11. 12 („welche du mir gegeben hast", könnte auch bedeuten: "welche er mir gegeben hat"); vor einem Pronomen, so diaçágun 52¹7; vor einer Postposizion, so deçánçat 90 12 . $97^{\prime} 4$. 359 '17. Dieses Letzte ist besonders bemerkenswerth, da -çat den Akzent zu tragen pflegt (8. oben 1) d) a)); aber deçan. cát bedeutet: „suf dass er habe".

b) auf dem -e-der Pluralendung (s. oben 1) a) a) bb)) vor einem silbigen Suffix, besonders vor -tan, z. B. ceinétan $66^{1} 15$, herfturétan $321^{\circ} 4$ (und étan noch sechs $\mathrm{Mal}$ im folgenden Vers), infolentiétan (und étan noch fünf Mal) 413'3. Sonst z. B. putéquin $137^{1} 30$, héquin (so) $164^{1} 22$, declarationéquin $\widetilde{I}^{\prime} 6$. Der Akzent dient zum Theil der Scheidung von den unbestimmten Formen: regionétan 327.10 ist "in den Gebieten", regionetan würde sein "in Gebiet". Ficotzétic $48^{\circ} 32$ hat den Akzent mit Unrecht(vgl.89:28); doch ist hier die Verwechselung ausgeschlossen, die unbestimmte und die pluralische Form dazu lauten ja ficotzetaric.

c) Vereinzeltes, Zweifelhaftes, Irrthümliches. Jenes Prinzip das sich in a) und b) offenbart könnte ja von L. in weiterem Umfang geltend gemacht worden sein; aber Schreibungen wie cietzóten $52^{\prime} 15$, ciesóten $145^{\prime} 39$, cioféla $84^{\prime} 17$, sachetéla $431^{1} 15$ steht die überwältigende Menge unakzentuierter Formen gegenüber. Am Ehesten liesse sich vermuthen dass er das -á. des Nominativs, des Aktivs, des Instrumentals in den Kasus mit 


\section{XCVII}

silbigem Suffix habe fortsetzen wollen in: Aitáren 354'19. CIII ${ }^{\top} 35$, aitári $136^{1} 12$, amáren $163^{1} 4 .^{2} 235^{\circ} 8$, Iaincoáren $\mathrm{B}$ VII ${ }^{1} 3$. Evir' 6 R. Aber gewöhnlich fehlt der Akzent: Aitaren $160^{\prime} 18$. $206^{1} 4.274^{\circ} 4.349^{\top} 11.427^{\circ} 3$, aitari $74^{\circ} 11.12 .311^{1} 24$ u. s. w., wobei ich ron den Verbindungen mit dem Possessivpronomen ganz absehe, da hier der Artikel stehen kann oder nicht (s. oben S. LXXXVI), also bere amaren zu bere amáren sich ebenso verhalten würde wie bere anayeren zu bere anayearen. - Ein Seitenstück zu dem oben erwähnten gendarmés bildet erréfa $340^{\circ} 14, \mathrm{er}$. réfac $316^{r} 22.320^{\circ}$, welches - ohne Artikel auch nach A. Chaho (BB 281) und Salaberry erres - dem franz. arrhe, gask. erro entspricht (kommt auch in der Bed. "Geissel" vor); Chaho kennt daneben die Form arrasa, das heutige Soulische hat arra. Wahrscheinlich ist eine Vermischung mit erresuc ,Ueberbleibsel" eingetreten. - Aus Versehen findet sich der Akzent auf der vorletzten Silbe mancher Wörter denen überhaupt kein Akzent zugedacht war, so bérac $268^{\mathrm{r}} 1$, bigáren $393^{1} 13$ (hier ist wahrscheinlich ein eingeschaltetes $r$ verlesen worden), çaréte $337^{\top} 13$, declára ${ }^{* *} \mathrm{IV}^{1} 13$, denuntiátzen $420^{1} 5$, érran $25^{1} 37$, eter. nála $\mathrm{CI}^{\circ} 5$, icéna ĩ $\mathrm{IV}^{\prime} \mathrm{b} 46$. In ein paar sollte er auf der letzten Silbe stehen, so anayéac $292^{\circ} 26$ (ein Mal, 306 ' 1 ist durch unsere Schuld 80 gedruckt, während der Urdruck anayeác hat), ani. málen $436^{1} 6$, celejtiála A IV 36 (Vok.). Aber écen $48^{\mathrm{r}} 33$ gehört nicht hierher; denn ecén (s. oben S. XCV u.) ist ebenso vereinzelt. Mit hámbat $26^{*} 54$ und hambát $82^{\circ} 30$ dürfte es sich kaum anders verhalten, obwohl der voneinander abweichende und zugleich ungewöhnlichere Gebrauch an beiden Stellen die Möglichkeit einer beabsichtigten Akzentsetzung zulässt.

3) Wo wir auf der drittletzten Silbe oder darüber hinaus einen Akzent finden, da liegt wohl meistens ein Versehen vor, so: alliángzzco $\mathrm{A} \mathrm{IV}^{2} 27$, contradictióneric $\mathrm{A} \mathrm{III}^{1} 11$, ezpáitute $63^{1} 18$, gáinean $\mathrm{A}^{\mathrm{\top}} 11$, gáitecen $67^{\mathrm{I}} 35$, paffiónea $32^{\mathrm{r}} \mathrm{U}$., sogar Jynágogán $64^{\mathrm{r}} 1$ mit zwei Akzenten. Indessen scheint duánaren $\mathrm{A} \mathrm{vI}^{\mathrm{r}} 13$ nicht ohn* Grund gesetzt zu sein (-a- \}-aka-), und so gehört vielleicht such in gaitualáric A IV 33 der Akzent eigentlich auf die drittletzte Silbe. Vgl. noch bércetan $\mathrm{Cv}^{1} 1$ (= bercétan $\left.\mathrm{Cv}^{1} 3\right)$ und défiréaric $\tilde{\mathrm{i}} \mathrm{Im}^{*} \mathrm{~b} 29$ (= defirétaric ?). Ganz allein stehen dürfte ézgeituztela $\mathrm{EvI}^{\mathrm{r}} 16$. Statt des Akuts habe ich ein $\mathrm{Mal}$ den Gravis bemerkt: duàn $359^{r} 17$, und als ganz überflüssig Màt. G vi'17 R.; drei Mal den Circumflex: Prophetâ $\widetilde{0} \mathrm{II}^{1} \mathrm{~b} 8$, Eliçân ơ II'b 15, refiumâ A III ${ }^{r} 36$ R.

Den Findestrich hat L. ebenfalls dem Französischen entnommen; die Angabe A. F. Didots (Observations sur l'ortho- 


\section{X்CVIII}

graphe française ' S. 59) dass der Bindestrich zuerst bei Nicot 1573 vorkomme, welche E. Koschwitz (Gramm. der neufranz. Schriftspr. S. 9) wiederholt, ist ganz unrichtig. Er findet sich, und zwar häufig genug, weit früher, so im franz. ${ }^{\text {sz }}$, z. B. foit-il, frappes-tu, tref-excellent, cevix-ci. Man kann mit gleichem Rechte wie als Bindestrich, dieses Zeichen auch als Theilungsstrich bezeichnen; es zerlegt was als ein Wort gedacht und gesprochen wird, für das Auge in die $z$ wei Theile aus denen es zusammengewachsen ist, es drückt eine etymologische Analyse aus. In Bexug auf die Trennung und Bindung der Wörter huldigen die baskischen Schriftsteller einer weit auseinandergehenden Praxis. L. ist hier vielleicht noch weniger als bei der Akzentsetzung mit sich ins Reine gekommen; es geschieht nicht selten dass er die gleiche Wortfolge nicht bloss in doppelter, sondern sogar in dreifacher Weise darstellt: als zwei getrennte Wörter, als Doppelwort mit Bindestrich und als êin Wort (zum Theil mit Lautverschmelzung). Ich gebe einen Ueberblick über die verschiedenen Gruppen von Fällen, die ich aber nur roh charakterisiere.

1) Substantiv + Substantiv :

a) kopulativ, so ama:femequin $2^{1}$ Ü., auch bei substan. tivierten Wörtern, so hartu-emaneuıco Kal. $1^{\prime} 9$ (disjunktiv: lucelaburrean $\mathrm{a} \mathrm{rv}^{1} \mathrm{~b}$ 30). Ferner:

$$
\begin{aligned}
& \text { aita-amén 18'21. aitamác 143'29. } \\
& 88^{\prime} 12, \text {-éc 10''27, 180'18, aitaméc } \\
& \text {-ác 103'41. } 119^{1} \quad 180^{\circ} 2.3{ }^{1} 20.181^{r} \\
& 56 \text {, -etara } 377 \text { '2. 22. 23, aitamén }
\end{aligned}
$$

b) apposizionell spezialisierend, so ama-guinharreba $13^{1} 14$, -aren $18^{\prime} 35$; u. s. w. Ferner: heredero quide $412^{2} 7$. foldado - quideari Anciano quide $414^{i} \quad 382^{12}$. 1, cerbitzariquideac 437'11. prefoner quideac 383 '23.

c) genetivisch spezialisierend, so gogo-handitaffiunaz ${ }^{2} \mathrm{v}^{1} 21$, parra-chori $18^{\prime} 29$, charpanter-feme $26^{\prime} 55$, bur-hegur $57^{\prime} 33$, ajto. omea $83^{1} 4$, borthal-sainari $89^{r} 34$. Auffallend oft erscheinen bei L. getrennte Wörter in diesem Verhältniss, so haour aitác $79^{\prime} 24$, itfas adar $263^{\prime} 39$, adin floreu $300^{\prime} 36$, belhar lilia $402^{\prime} 10$. 410'24, oin hatzey $411^{\prime} 21$, bilo igurduratan $412^{\prime} 3$, carrets hotfa $441^{\prime} 9$, burdin berga 454'15; in manchen Fällen war eine engere Verbindung deshalb nicht möglich weil das erste Wort selbst wieder näher bestimmt war, nur hätte man dann 
eben den Genetiv erwarten sollen (man vergleiche das noch merkwürdigere dohain fpiritual guthiciofo $308^{1} 1.309^{5} 12$ für d. Spiritualén g., wie kurz vorher $307^{1} 31$ dohain excellentenén guthiciofo steht). Das Vorkommen des Genetivs neben dem Nominativ in denselben Wortfolgen (wie fuaren garra beçala $431^{\prime} 14.433^{r} 18=$ Ju-garra beçala $454^{r} 12$ [ fu flamma $384^{1} \mathrm{~T}$ ]) brauche ich hier nicht weiter zu verfolgen. Auch zwei Substantive stehen in genetivischem Sinn ohne Bindestrich vor einem dritten, so aker edo aretze odolez $393^{\prime} 12$, chirula, eto trompetta foinularién $453^{-22}$. Ferner:

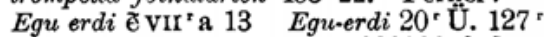

$($ egun +$)$. 31. $222^{1} 26.252^{\circ} 6$. gau-herdit $\tilde{a} \quad 49^{\circ} 6$, gantherdi $247^{\prime} 7$, -itan gau-erditan $126^{\circ} 5 . \quad 89^{\prime} 35$.

heufcal herriä* ${ }^{*} I^{1} 15$. Heufcal - herrian

* VII 9 .

Vgl. noch:

vme-vncia $102 \times 23$. vmunci $272 \times 19$.

auri-nombrea Kal. I'3. Auri nöbrea Kal. $\mathrm{II}^{\mathrm{*}} 2 .{ }^{2}$ '.

Besonders stelle ich die Verbindungen deren zweites Wort ein Verbalsubstantiv ist, weil sich hier leicht die verbale Konstrukzion fortsetzt und auch in der Schreibung abspiegelt. So sehen wir getrennt geschrieben vzta biltzaleac $25^{\wedge} 39$, gaitz-erideile $267^{\prime} 30$, gaitz erizle $381^{\prime} 3$, hobe erizle $377^{\circ} 4$, odol iffurtze $394^{\mathrm{r}} 22$, guiça pe/cadore $66^{\mathrm{r}} 19.61^{\mathrm{r}} 17$. Ferner:

Gaizqui eguile $\mathrm{I}^{1} \mathrm{~b} 24$. gaizqui-eguile $199^{1} 30$. gaizquiguilez $411^{\circ} 12$, -lén 411'14, (gaiz. qui-|guile $155^{1} 32$, gaizquiguile $\mathrm{Crv}^{\mathrm{r}}$ 26.32 (vgl. templetchoguile 246r24).

guiça-erhailey $456^{1} 8$. guicerhailebat $211^{1} 14$, -le 264 4 .

guiça-erhaitecác gricerhailecaz 267'29. $337^{1} 21$, tequetaric $441^{1} 21$.

Legue egarlea $406^{1} 12$, Legue-eçarle ** $\mathrm{VII}^{1} 35$, -leric ${ }^{* *} \mathrm{II}^{1} 37$, -lea- -lea $\mathrm{AII}^{1} 24$. ren DVII ${ }^{1} 14 \mathrm{R}$.; baque ecarlea $* * *$ III $^{\mathrm{r}} 34$.

vngui eguitez $411^{\ulcorner} 15$.

vnguiguitez $338^{1} 9$ vnguiguile 151'25, -lén 411²14. 
2) Substantiv + Adjektiv. So :

dohain gaiz $149^{1} 23$, Dohain-gaitz $47^{\prime} 19$. dohaingaitz 88'17. dohain $\mid$ onezco ${ }^{* * *}$ dohain-onetaco ${ }^{*} \mathrm{VII}^{*}$ dohainontaffunez ${ }^{* *}$ $I^{1} 35$. 29.

bothere gucitacoa bothere - gucitacoá 448'3. $\mathrm{Cr}^{2} 38,-c o$ $\mathrm{BVIII}^{1} 8$. 36. $\mathrm{CI}^{\mathrm{r}}$ 30. $39 \mathrm{R}$. $443^{1} 17,-c o$ Brr $31, \quad 436^{r} 8$. -coaren BII ${ }^{\mathrm{3}} 37$. $\mathrm{CI}^{\mathrm{r}} 1$.

haice contra $27^{1} 24$, haice-contra $73^{\mathrm{r}} 48$ haice contreac (contra ist dem $261^{\prime} 4$. span. contraviento als Adj. entnommen).

bajja eztiz $4^{\circ} 4$.

Vgl. vana-gloria $338 \mathrm{r} 26$.

bafSezti $60^{\prime} 6$.

3) Substantiv + Postposizion. So hoguen-gabeac 21.7, letra-gabeac $213^{\mathrm{r}} 13$, Haourra-danic $78^{\prime} 21.378^{\circ} 15$. Ferner: ezcồdu gabe 298'11, ezcondu-gabey $298^{1} 8$. -beac $299^{1} 34$.

hatsean-danic $420^{r} 1$. hatfeandanic $179^{\mathrm{r}} 44$. $421^{1}$ 13. $422^{\text {r } 24 . ~} 194^{1} 27$ u. s. w.

hoguen gabe $\mathrm{CIV}^{\mathrm{r}} 34$. hoguen-gabeac $21^{\circ} 7$, -be CIV ${ }^{2} 29$, -betan $\mathrm{DrV}^{1} 17$, bearen Fvir'2.

Orduan-danic 6¹7. Orduandanic $31^{1} 21$. finhefte gabea $33^{\mathrm{r}} 17$. finhefte-gabe $204^{1} 27$. $78^{1} 19$.

Hier schliesse ich noch an finhejte-dun $204^{1} 27$, fundament-dunaren $397^{\mathrm{r}} 10$, obwohl -dun im Grunde nichts Anderes ist als duen "welcher hat"; gewöhnlich wird -dun mit dem Substantiv fest vereint, z. B. Hartzedun $115^{1} 41$.

4) Verb + Hülfsverb im Imperativ. So:

Iar adi $43^{1} 44.148^{\prime}$ Iar-adi $87^{\mp} 36$. 42 u. s. w.

beha-eşac 121'38. Beheşac $211^{\prime} 4$. A vi $^{1}$ fcriba eçac 138\%7 A viI ${ }^{1} 6$.

u. s. w.

Wenn der Verbalstamm auf $a$ ausgeht, und die Imperativform mit $a$ anhebt, so tritt besonders gern Verschmelzung auch in der Schrift ein : batheyadi, behadi, diligentadi, emendadi, lehiadi. Ebenso beguirauc, beguirauçue, wo beguira aber nicht als Radikal $\mathrm{zu}$ beguiratzen anzusehen ist (dann stünde beguira eçac; vgl 
269'25. 421'3), sondern adverbial (,hab es im Auge" $)$. Auch das häufige gogoaugue werden wir in gogoa augue, nicht in gogo aucue, trotz gogo-|auçue $314^{r} 10$, aufzulösen haben (gogoa ist hier Substantiv, wie in gogoa emaiten $134^{\circ} 1$ u. 8. w., aber Radikal zu gogoatzen [gogoatu $\mathrm{GV}^{1} 38$ ] in Gogoa eçac $359^{\prime} 17$; vgl. 228'6.290'17). Hierher gehört auch mehatchaçala 429 9 für mehatcha agala (vgl. oben S. XLVIN). Andere Verbindungen kommen noch seltener vor, so erraitê-bainuẽ 160 '15, vkiä-dugu $324^{\circ} 18$, içã-|çaizcan $61^{\prime} 18$ (içan ģaizcan 61'20), behar-|da 247'36, hartzế-|da $\mathfrak{a ~ v}^{1}$ a 5 ; in den letzten Fällen kann auch der Bindestrich als Theilungsstrich gefasst werden. Als völlige Verschmelzung ist zu bemerken arigan 291'15.

5) irgend welches Wort + -ere, so nic-ere, baditez-ere, orain-ere. Dies ist der einzige wirklich regelmässige Gebrauch des Bindestrichs, im Vergleich zu dem sein sonstiges Vorkommen sls selten und willkürlich erscheint. Nur ganz ausnahmsweise sind die betreffenden Wörter in eines geschrieben, so guriere $237^{r} 8$, nehorere ${ }^{* *} \mathrm{IV}^{\mathrm{r}} 29$, norere $281^{\prime} 13$; in badere $185^{\mathrm{r}} 25$. $\mathrm{CVII}^{\prime} 38$. DVI'31, badituztere ${ }^{* *} \mathrm{II}^{2} 12$, halere ${ }^{* *} \mathrm{VII}^{1} 26$ ist dabei der Vokal vor e geschwunden. Für batere hat L. regelmässig batre. Etare steht gewöhnlich für eta are; Beides nahe nebeneinander $\mathrm{Cn}^{\prime} 10.15$.

6) Nomen + bat. Die Regel bei L. ist die dass der unbestimmte Artikel mit dem vorhergehenden Worte zusammengeschrieben wird, wobei Angleichung des $n$ stattfindet (z. B. guiçombat $21^{\prime} 10$, orembat $53^{\prime} 40$, ombat $90^{\prime} 6$, cembat $93^{\prime} 7$, suembat 219r24). Der Bindestrich kommt aber doch nicht selten vor, so voz-bat $60^{1} 11$, egun-batez $63^{1} 23$, egun-baten $264^{\prime} 13$, guiço-bat 109'18. '225'33, guiçon-bat $111^{\prime} 6.160^{\prime} 6.166^{1} 29.168^{\prime} 5$. 175'23. $224^{\prime} 12$. '239'9. '258'14, guiçō-batez $273^{\prime} 12$, cen-batec $115^{1} 37$, cen-bat $261^{1} 6$, iaun-bat $167^{1} 46$, gaichtaguin-bat $200^{\circ} 40$, bildots-bat $223^{\prime} 32$, den-bat $262^{*} 14$. 390'19. Hat bat ein Kasussuffix, 80 löst es sich leichter von dem vorhergehenden Wort los (vgl. arbat $187^{\prime} 14$, ar-baten $187^{\prime} 15$ ), und völlige Trennung der Wörter scheint fast nur in diesem Falle einzutreten, z. B. aitafamilia batequin $37^{1} 1$ (aitafamiliabat $41^{\circ} 33$ ), regue batequin 42'2, Guiçon batec $40^{\prime} 28$, berce bati $122^{\prime} 59$. Ber-bat, ber-batez, ber-batetaco ist die herrschende Schreibung, die einerseits berbat $291^{\prime} 10$. $307^{\prime} 25$, anderseits ber batetaric $280^{\prime} 21.302^{\prime} 3$, ber batez $306^{1} 13$ neben sich hat; in Spiritual-ber batetaric $303^{\prime} 4$ wäre es möglich dass der Bindestrich am falschen Platze stände. Man bemerke noch Nabufsibi ivir'b12 nach Analogie von nabu/sibat; an beiden Textstellen hat das Zahlwort die gewöhnlichere Stellung: $m i n$. 


\section{CII}

7) Zahlwörtermultiplikazion. Die mit 20 zuzammengesetzten Zahlen erscheinen immer in einem Wort: berroguey, hiruroguey, lauroguey, jeioguey $\left(\widetilde{\mathrm{a}} \mathrm{vI}^{1} \mathrm{~b} 15\right.$; wegen $-i$ - $\mathrm{s}$. S. XXVII), von den mit ehun zusammengesetzten ber-ehun immer mit Bindestrich, ferner hirur-ehũ ${ }^{*} \mathrm{VI}^{\mathrm{r}} 14$, hirur-ehun ${ }^{*} \mathrm{VI}^{\mathrm{r}} 18.90^{\circ} 5$ neben hiruriehun $187^{\top} 5$, borz-ehun $11^{\prime} 41$ neben borz ehun $311^{\circ} 6$, Jey ehun $446^{1} 18$; die mit milla zusammengesetzten werden getrennt geschrieben, auch hamar milla, nur $35^{\text {r22 }}$ hamar-milla.

8) Vereinzeltes und Irrthümliches. Bindestrich bei Reduplikazion: ber-bera $27^{1} 23$. $171^{\prime} 15$, bere-berez ${ }^{*} \mathrm{vuI}^{\top} 34$, behin-behin F VIII '15, zwischen Prädikat und Kopula : gende-eztenaz $281^{1} 19$, zwischen gen. Substant. und Adj.: on-beharrén ${ }^{* *} \mathrm{VII}^{1} 30$ (on behar B viI' 35 ), zwischen obj. Adj. und Verb: eder-ets G vII' 20 (neben gaitzet/i u. s. w.). Man bemerke ferner:

baina aitzitic $344^{r} 28$. baina-aitzitic BIV ${ }^{r} 25$. bainaitzitic $18^{r} 28$.

D vir36. Fv'37. FiII'11. vII ${ }^{r} 39 . \quad 140^{\prime} 8.281^{\prime} 18$. $286^{\prime} 13$.

In niri-baldin $30^{\circ} 2^{\circ} 16$ und bainante-eta $386^{1} 9$ ist der Bindestrich für den Beistrich verdruckt, in luc- ã virI' a 37 für den Punkt, in Loctenent general. ${ }^{*} \mathrm{VI}^{\mathrm{r}} 31$ hat er seine Stelle gewechselt, in bere-odolaz $401 \times 12$ ist er ganz gedankenlos angebracht. $E z$ wird regelmässig mit dem Verb und gewissen andern Wörtern (wie deus, eta u. s. w.) fest verbunden; doch fehlt es nicht an Abweichungen; so ez gara 313r51, ez-tuún A viI' 4, ez-deus, ez-|deujago ${ }^{*} \mathrm{VI}^{1} 13$, ez eta ${ }^{* * *} \mathrm{I}^{\mathrm{r}} 23$, ez-eta $\mathrm{F}$ VIII ${ }^{\mathrm{r}} 23$. Auffällig ist auch mijericordia-gatic $\mathrm{G} \mathrm{III}^{\mathrm{r}} 22$, unmittelbar nach onta/fiinagatic. Bei manchen Worttrennungen können wir im Zweifel sein ob sie rein mechanischer Art sind oder doch eine Analyse zu Grunde liegt, z. B. gortzi garreneco $351^{5} 5$, ez eztatu ${ }^{*} \mathrm{VIII}^{1} 6$, berant eften ${ }^{* *} \mathrm{III}^{*} 24$.

Ein Gebrauch des Bindestrichs hat einen von dem sonstigen abweichenden Zweck. Es wird nämlich die Kasusendung abgetrennt um die Aussprache der Schlusskonsonanten in fremden Eigennamen und doch auch deren Schreibung unberührt zu lassen - ein Verfahren das auch den Slawen nicht unbekannt ist. Aber nur gewisse Konsonanten erheischen den Bindestrich, wie schon ein flüchtiger Blick auf die Geschlechtstafeln von Bl. 1 f. und 105 ergeben wird. Ohne Weiteres ist klar warum L. Ifaac-en, Ifaac-equin, Ifaac-i, Marc-en, Phalec-en, Sadoc-ec schreibt; auch warum Grec-ez 156'38. 441 ' 11 . ã II ${ }^{x} \mathrm{~b} 33$, obwohl er sich anderswo nicht scheut in der Schreibung dieser Wortform den Franzosen zu folgen: Grecquez 201'20. a ${ }^{\prime \prime I}{ }^{1}$ b 30 (wie auch Grecquic $251^{1} 37.335^{r} 28$ ). Auch ein Appellativum behandelt er so: lac-|era $17^{\prime} 23$, lac-era $118^{\mathrm{r}} 33$; zu 
lac-agatic $\mathrm{a}_{\mathrm{IV}} \mathrm{b} 21$, aspic-aren $270^{\prime} 13$ aber ist kein Grund ersichtlich. Da in der Sprache L.s ch im Auslaut höchst selten ist, so war wenig Gefahr dass die Basken in Saruch, Lamech, Henoch den Zischlaut hören liessen; in Saruch-en, Lamech-en, Henoch-en, Henoch-ec war der Bindestrich nicht so überflüssig. Wenn L. aber nun auch Dauid-en, Dauid-ec (Dauidec-ere $271^{\prime} 6$, um nicht zwei Bindestriche zu haben), Dauid-i, Obeden, Obed-ec, Abiud-ec, Eliud-ec, Iared-en und Iacob-ec, Iacob-en, Iacob-equin, Iacob-i, Aminadab-en, Beelzebub-en schreibt, so verhält sich die Sache einigermassen anders. Hier galt es gerade die baskische, nicht die fremde Aussprache zu wahren; der Baske kennt die Media im Auslaut nicht, er spricht Dauit, Iacop; in Dauiden, Iacoben würde er die Media gesprochen haben, und um ihn daran zu hindern, schaltete L. den Bindestrich ein. Axulars Schreibung Dauitec neben Dauid ist ganz unzweideutig. In Appellativen verfährt $L$. auf die gleiche Weise: paillard $296^{1} 11^{-}$- paillartéc $297^{1} 10.344^{1} 5$, paillartequin $296^{1} 10$, paillartac $\mathrm{B} 1 \mathrm{II}^{1} 19$; aber bei paillardá $398^{\circ} 31$, paillardatzera $433^{\mathrm{r}} 20$, paillardiça- $8^{\mathrm{r}} 32.29^{\circ} 19.344^{\prime} 3 \mathrm{nahm}$ er das $d$ unmittelbar aus franz. paillarde, paillarder, paillardise. Auch Seth-en $105^{1} 38$ ist begreiflich (allerdings Muathen 105.26), da im Auslaut th soviel ist wie $t$; aber Lot-en 141'28. 32, Azot-en $223^{\circ} 40$ (hingegen Matthaten 105'24. 29) nicht. In A/er-en 102'36, Iupiter-en $235^{\mathrm{P}} 13$ würde die Trennung begründet sein (Axular schreibt Thamar, Thamarrec); und zwar umsomehr als A/eren, Iupiteren auch die Genetive von Aje*, Iupite* sein könnten (aber daneben finden wir Eliezeren $10 \bar{j}^{\mathrm{r}}$ 29). Bei Capernaum-en $61^{\mathrm{r}} 21.107^{\mathrm{r}} 23$ könnte man an die französische Aussprache denken, aber es steht vier oder fünf Mal so oft Capernaumen und Capernaumera. Phanuel-en $102^{1} 36$ und Cis-en $233^{\circ} 21$ halte ich für bedeutungslos gegenüber Zorobabelen, Henojen u. s. w. In Chanaun-era $218^{1} 11$ sollte vielleicht der Bindestrich verhüten dass man $e$ zum Namen selbst zöge; vgl. Adam-etan ã vIII'b 37 $=$ Adamtan $311^{1} 22$ (wie Genezaretheco = Genefarethco oben S. XXVIII). Core-erén 429r11 ist eine Vermischung von Core-en und Core-ren.

Der Theilungsstrich welcher am Ende einer Zeile steht, könnte mit gleichem oder mit besserem Rechte Bindestrich heissen; er bindet die weit auseinander gerissenen Theile eines Wortes. Er wird sehr oft bei L., ebenso wie in den französischen Drucken jener Zeit, weggelassen; die einzelnen Bogen verhalten sich hierin zum Theil recht verschieden, z. B. fehlt er im N.T. in $a$ kein Mal, in $b$ zwei Mal, in $A 9 \mathrm{Mal}$, in $B$ 13 Mal. Natürlich bei Halbzeilen weit häufiger, z. B. auf 


\section{CIV}

dem einzigen Blatt ã II 17 Mal. Selbst wenn wir die Vertheilung des Stoffes auf die Zeilen nehmen wie sie ist, werden wir oft keine wirkliche Nöthigung zum Weglassen des Theilungsstriches erblicken, und dieser Umstand erschwert uns in gewissen Fällen das Urtheil darüber ob L. zwei oder ein Wort hat schreiben wollen. Es scheint dass man sich den Theilungsstrich da gern ersparte wo er zugleich den Bindestrich vertreten haben würde, z. B. bur heçur $\mathfrak{a}^{x} \mathrm{I}^{r} \mathrm{~b} 16$ (neben dreimaligem bur-hecur), wie im franz. ${ }^{77}$ : Leue $\mid$ toy $2^{1} 13$ (neben Leue-toy $3^{r} 20$ ). Aus der Art der Wortabtheilung lassen sich kaum Schlüsse auf die Aussprache ziehen; höchstens dass die Trennung von ai oder au (wie ga-|ichtoac $22^{\prime} 35$, compa|inia $109^{1} 29$, Ia|incoari a $\mathrm{vI}^{\mathrm{x}} \mathrm{b} 6$, Ia|incoaren ã $\mathrm{VI}^{1}$ a 33 , ba-|itu $\widetilde{\mathrm{a}} \mathrm{Im}^{\prime} \mathrm{b} 33$, ira-|unguiric $\mathrm{A} \mathrm{I}^{1} 11$, dra-|uean $\mathrm{A} \mathrm{II}^{\mathrm{r}}{ }^{15}$ ) gegen die monophthongische Geltung dieser Verbindungen zeugt, welche im Französischen, wenn ich nicht irre, die Trennung verhindert; sie zeugt aber gewiss nicht gegen die Einsilbigkeit, wie man nach dem allgemeinen Grundsatz für die Zeilenbrechung voraussetzen sollte. Man bemerke: farge-|antac $131^{1} 58$, conjeil-|luan $217^{1} 15$, Veil-|latzera $247^{\circ} \mathrm{U}$. (wie franz. efveil|lé u. s. w.), Allica-|nçaco 393.4, Do-|ctrina $400^{1}$ Ü., rejpe-jctuz ${ }^{* *} \mathrm{III}^{1} 31$ (wo aber zu bedenken ist dass im Urdruck $c t$ ein Zeichen bildet). Ein falscher Theilungsstrich cer-|den $\mathrm{C}$ VII ${ }^{\mathrm{r}} 22 \mathrm{R}$.

Der Gebrauch der eigentlichen Interpunkzionszeichen bei L. entspricht im Wesentlichen dem den die gleichzeitigen französischen Drucke zeigen; der Beistrich vertritt unsern Doppelpunkt vor der direkten Rede und der Doppelpunkt unsern Punktstrich, der damals überhaupt noch nicht vorkommt. An einer Stelle, 170r39, haben wir ihn gesetzt, da im Urdruck sich, durch irgend ein Versehen, ein derartiges Zeichen herausgestellt hat (der Punkt ist aber nur schwach). Aus der Eigenthümlichkeit des Baskischen „den Wagen vor die Ochsen zu spannen", das heisst einem "Substantiv oder determinativen Pronomen den dazu gehörigen Relativsatz oder eine längere attributive Bestimmung mit - $c o$ vorauszusetzen, gehen nun aber doch Setzungen des Beistrichs hervor die ausdrückliche Erwähnung erheischen. Ich beschränke mich darauf sie und ebenso die nicht seltenen Abweichungen mit je einem Beispiel zu belegen:

1) der Beistrich wird $n a c h$ jenem Substantiv oder Pronomen gesetzt wenn nicht das Verbum finitum, sondern das Prädikat oder Subjekt oder eine adverbiale Bestimmung folgt: Cuec recebitzen caituztenac, ni recebitzen nau $18^{1} 40$; hingegen arbore fructu onic eguiten eztuen gucia piccatzen da $4^{\prime} 10$. Aus. 
nahmen: gure jperança şueçaz duguna fermu da $315^{1} 7$ - hare. quin ioaiten ciraden guiçonac, gueldi citecen icituric $2244^{\circ} 7$ (vgl. cegoenac, guen $437^{\prime} 5$ neben cegoenac ģuen $437^{\circ} 2$ ).

2) der Beistrich wird vor den Relativsatz oder die sonstige attributive Bestimmung gesetzt wenn das Verb vorausgeht, aber nicht unmittelbar: conta ietzoçue Ioanne/ï, ençuten eta ikuften dituçuen gauçác $19^{\mathrm{F}} 4$; hingegen has cequién hunela reprochatzen haren verthuteric anhitz eguin içan cen hiriey $20^{2} 20$. Ausnahmen: parti şaitezte eneganic iniquitate eguiten duçuenóc $12^{\circ} 23$ - recebituren duque, Iaunac hura maite duteney promettatu drauen vicitzeco coroá $402^{\prime} 12$. Aber in diesem Fall rechtfertigt sich doch wiederum die Setzung des Beistrichs, die ja schliesslich nur dem Zwecke der Deutlichkeit dienen soll. Der Beistrich deutet auch das Fehlen der Kopula an - wie im Russischen der wagrechte Strich -, z. B. ceinén Iaincoa, Jábela $352^{*} 19$. Er trennt auch Subjekt und Prädikat, z. B. Cergatic deitzen

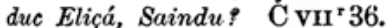

Einer ganz besonderen Verwendung des Beistrichs will ich hier gedenken. Van Eys Dict. S. 344 ${ }^{\circ}$ bemerkt zu dem Suffix ta ${ }_{n}$ nachdem " (ikusita, après avoir vu ou ayant vu; edanda, ayant bu), dass Leiçarraga und Pouvreau für dieses -ta noch eta schreiben, z. B. hartan farthu eta eridenen ducue afto-vme arbat Luk. XIX, 30. Es unterliegt keinem Zweifel dass es sich hier um eta "und" handelt, und unkere Sprachen gewähren dafür ausreichende Analogieen (8. z. B. Dodgson Rev. de ling. XXXII, 327). Was den thatsächlichen Gebrauch anlangt, so ist die Angabe von van Eys nach zwei Seiten hin zu ergänzen. Erstens spricht und schreibt man auch heute noch in weitem Umfang eta, z. B. Hitz hauk erran eta, lurrerat erortzen da Dasconaguerre (BB $377^{\prime}$ ) S. 9, Ibaya hertsiena zen tokirat heldu eta Ganichek beiratzen du ur zolarat ebend. S. 140; es kann auf das eta auch noch eine Bestimmung zum Partizip folgen, so eskerrak bihurtu eta murde Belzunzeri, "guazin" dio printsesak Ganichi ebend. S. 38 f., Ganich inguru behatu eta nihork ikusten othe duen, ilkhitzen da ebend. S. 140. Und zweitens, schon bei L. war die Bedeutung dieses eta verdunkelt, er setzt nämlich den Beistrich danach, den van Eys in jenem Zitat $\left(145^{r} 30\right.$, und so $75^{1}$ 6. $117^{1} 27 . \mathrm{Cv}^{\mathrm{r}} 21 \mathrm{u}$. s. w.) nicht wiedergegeben hat.

Die Interpunkzionszeichen fehlen zuweilen wo sie unbedingt nothwendig wären. So der Punkt hie und da aus Platzmangel, indem der letzte Buchstabe des Verses ganz an das Zeilenende zu stehen kommt, z. B. $26^{*} 45$ (so auch das Fragezeichen z. B. D $\mathbf{v I}^{2}$ 1). Doch auch ohne dies am Versschluss, $80286^{\prime} 8.352^{1} 10.429^{\circ} 11$. In der Mitte des Verses am 


\section{CVI}

Zeilenschluss $353^{\prime} 21$; ohne dies z. B. $290^{r} 16$. A m'33. vi'18. BII ${ }^{1} 34$. Unter dem starken Einfluss der Raumverhăltnisse wird in den Randschriften der Punkt (den ich wohl auch in seiner nichtsyntaktischen Rolle hier abthun darf) sei es nach den Zahlen sei es nach den Namen oder Wörtern unzählige Male nicht gesetzt; aber er wird nach diesen auch da wo sie nicht abgekürzt sind, sehr oft oder vielleicht ebenso oft gesetzt, so Marc., Ioan., Luc., Lehen., Berriz., sogar nach eta kommt er vor, so ** III'31. 3059. 357'8, auch ă $I^{1}$ a 26 . Die Zahlen der Kapitelüberschriften pflegen keinen Punkt nach sich zn haben; zuweilen steht er aber auch hier: $292^{1}$. 301' u. 8. w. Der Beistrich ist verhältnissmässig selten weggelassen (vgl. vorh. S.), z. B. 114' 22 (nach chahutzen), 4325 (nach ezpere; vgl. $\left.433^{\circ} 16\right)$. Falsch gesetzt, das heisst wo überhsupt kein Zeichen statthat, ist sonst der Punkt in äusserst wenig Fâllen, so $207^{1} 22$ (nach dadĩ), 328'33 (nach itzur; im L., nicht im St.), 367' Û. des Briefs (nach Epifola), a vir'c 16 (nach membroz), A vIr 39 (nach eure). In sehr vielen aber der Beistrich, z. B. $73^{1} 4$ (itzultzen, diradenean), $169^{\prime} 30$ (nauen, Aitaren), $264^{\prime} 4$ (itfaffotic, emparaturic), 386'1 (baita, Iefus), i v'b 18 (bere, buruac). Vereinzelt der Doppelpunkt, so E vir ${ }^{1} 12$ (vor baitu; ein Doppelpunkt steht darunter in der folgenden Zeile). Nur selten hat ein Zeichen seinen Platz verändert, so ist $380^{1} 15$ der Beistrich vom Ende der 4. Zeile (nach dituc) auf das der 5 . Zeile hinuntergeglitten (nach deus), so $32 S^{\prime} 9$ der Doppelpunkt vom Ende der letzten Zeile (nach graticiz) auf das Ende der Signatur (ecen). Manchmal sind zwei Zeichen zusammengekommen: Matth..26 190'21 R., ioan... 1s б $\mathrm{ru}^{\circ} \mathrm{a} 10$, guehien, . luc è $\mathrm{IV}^{\circ} \mathrm{b} 43$, Profelytoa, hambat (so nahe im Urdruck) $\tilde{a} v^{\prime} a 27$. Beispiele für die nicht allzuhäufigen Vertauschungen der Interpunkzionszeichen untereinander: . statt , 241'6 (nach ceudela), 304' 31 (nach ba-|duçue), $395^{\prime} 8$ (nach gainean) . statt ? $170^{\prime} 47$ (am Schl. d. V.), 321 114 (am Schl. d. V.), B virI'9 (am Schl.) , statt . $222^{1} 24$ (am schl. d. V.), : statt , $303^{1} 21$ (nach çaratezte; es geht : voraus) ? für, 164'12 (nach ezpadituçue; es folgt ?) ? für : ${ }^{* *} \mathrm{VI}^{\prime} 1$ ) statt , 400'21 (am Schl. d. V.; es geht ) voraus). Natürlich ist dann und wann eine verschiedene Interpunkzion möglich; so schreibt L. $275^{1} 7$ : Guertha eztadila. Aitzitic, aber $276^{\circ} 13$ dieselben Worte: G. e.: a. Wenn $79^{1} 43$ hura: hobe duc hire, aber 80.45 und 47: hura, hobe duc hire steht, so werden wir doch nicht trotz dieser Majorität mit Dodgson Rev. de ling. XXXI, 292 sagen: "Les deux points : sont une faute de P. Hautin." Der Doppelpunkt hier entspricht durchaus seinem sonstigen Gebrauch bei L.; auch die franz. ${ }^{63}$ und ${ }^{17}$ und die Vulgata 
haben ihn an allen drei Stellen. Statt trublatua Bada: behar da B vir'27 wird zu schreiben sein tr., bada behar da.

Die Abweichungen des Abc ${ }^{*}$ vom TB in den ihnen gemeinsamen Theilen (s. S. IX) will zwar Vinson, der beide Wort für Wort verglichen hat, BB S. 44 ff. verzeichnen, aber thatsächlich enthält dies Verzeichniss nur einen kleinen Theil der Abweichungen und ist selbst durch etwa anderthalb Dutzend Druckfehler entstellt. Vielleicht habe auch ich nicht völlig ausgeschöpft; die Vergleichung ist, da die Texte sich in ihrer Druckform nicht miteinander decken, sehr ermüdend, und es war mir nicht allzuviel Musse dazu gewährt.

L. hatte bei dem Wiederabdruck zweierlei vor Augen: die Verbesserung des Textes und eine auf wenig Punkte beschränkte mundartliche Retouehierung.

Ich stelle zunächst die Druckfehler und Schreibvarianten des TB, welche im Abc verbessert sind, nach ihrer räumlichen Folge zusammen und verweise wegen des Genaueren auf die frühere Einordnung und Würdigung derselben. In diesem ganzen Anhang bezieht sich immer die erste Anführung auf das TB, die zweite auf das Abc.

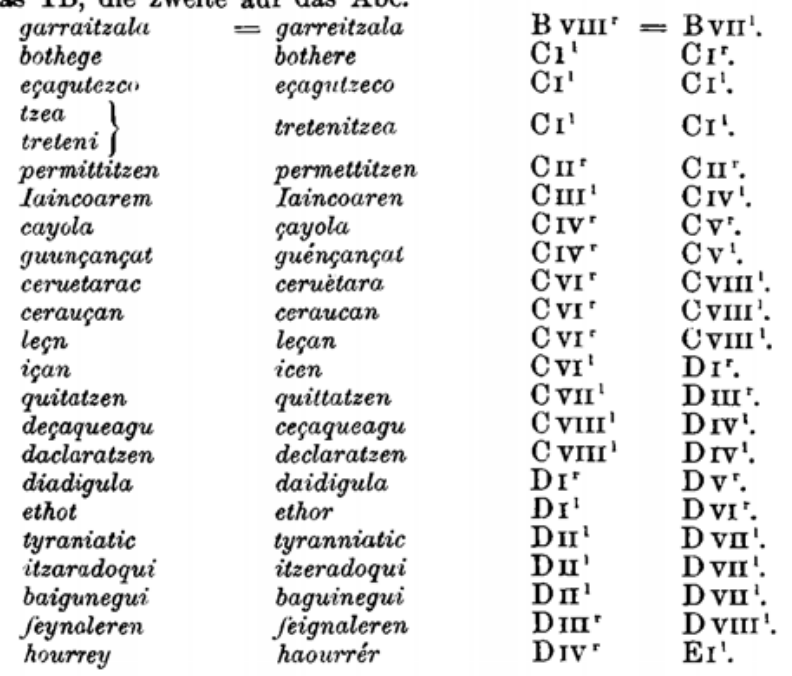




\section{CVIII}

\begin{tabular}{|c|c|c|}
\hline $\begin{array}{l}\text { entetenitzeagatic } \\
\text { beldurequin } \\
\text { othoit } \\
\text { BBaina } \\
\text { badietzacogu } \\
\text { drauģa } \\
\text { babitationea } \\
\text { dif-|famatzera } \\
\text { cerbi-|zatzen } \\
\text { feygnale } \\
\text { cutenean } \\
\text { onduan } \\
\text { mendicaturic } \\
\text { vkaitara } \\
\text { eliçate } \\
\text { ez eliçate } \\
\text { delaratione } \\
\text { çarri } \\
\text { infimitatearen } \\
\text { baguindu } \\
\text { ikus garria } \\
\text { batheiatzen } \\
\text { aitzetic } \\
\text { gaituala } \\
\text { ecaque } \\
\text { eçeinere } \\
\text { cerbait } \\
\text { paffutara } \\
\text { canonicotan } \\
\text { miracaluric } \\
\text { gathibatuac } \\
\text { aborritzen } \\
\text { vra }\end{array}$ & $\begin{array}{l}\text { entretenitzeagatic } \\
\text { beldurrequin } \\
\text { orhoit } \\
\text { Baina } \\
\text { badietzogu } \\
\text { drauca } \\
\text { habitationea } \\
\text { diffamatzera } \\
\text { cerbitzatzẽ } \\
\text { jeignale } \\
\text { çutenean } \\
\text { ondoan } \\
\text { mẽdecaturic } \\
\text { vkaitera } \\
\text { ezliçate } \\
\text { ez ezliçate } \\
\text { declaratione } \\
\text { eçarri } \\
\text { infirmitatearen } \\
\text { baguendu } \\
\text { ikuzgarria } \\
\text { batheyatzen } \\
\text { aitzitic } \\
\text { gaituela } \\
\text { eçaque } \\
\text { eceinere } \\
\text { cerbait } \\
\text { pauffutara } \\
\text { canonicoetan } \\
\text { miraculuric } \\
\text { gathibatua } \\
\text { abhorritzen } \\
\text { hura }\end{array}$ & 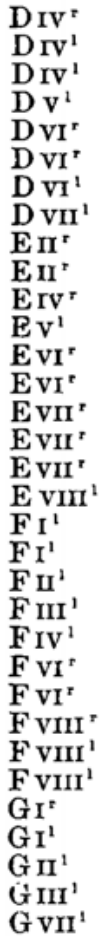 \\
\hline
\end{tabular}

Verschiedenes ist im Abc geblieben was der Abänderung bedürftig erscheint, so:
Apoftulu alchatzen foffagu
${ }^{\text {B viri }}{ }^{1}=$ Bvirr $^{r}$.
Ev ${ }^{r}$ LII.

Aber es lässt sich nicht in allen Fällen die Absicht L.s errathen; so mochte er dauritzan $\mathrm{F}$ VII ${ }^{r}=\mathrm{K}{ }^{*}$, extrordinario $\mathrm{G} \mathrm{vI}^{1}=\mathrm{Lv}{ }^{r}$ als gute Schreibungen betrachten. Auch handiraffun $\mathrm{FVIII}^{\mathrm{r}}=\mathrm{K}_{\mathrm{II}}{ }^{1}$, handiraf/una $\mathrm{FVIII}^{1}=\mathrm{K}^{\mathrm{III}}{ }^{1}$ sind geblieben. Auf der andern Seite finden sich nun eine Reihe neuer Druckfehler und Inkonsequenzen: 


\section{CDX}

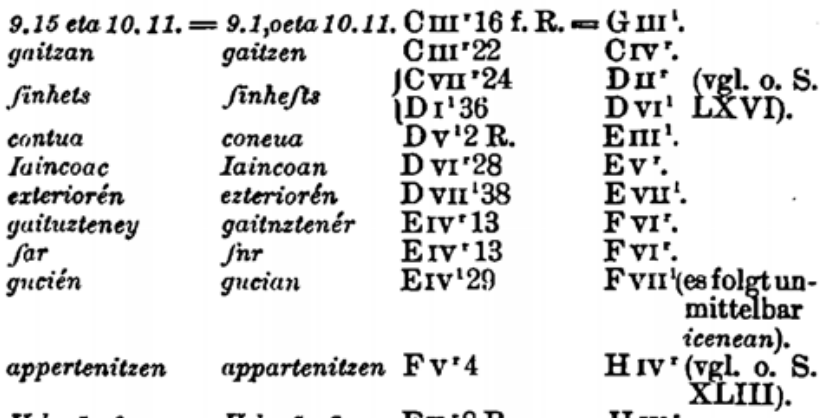

Heb. 5. 6

Batheyatzea

Batheiatzea FVI'18

$\mathrm{H} \mathrm{VI}^{\prime}$ (vgl. o. S.

jcandaloén jeandoloén $\mathrm{FvI}^{121} \mathrm{H} \mathrm{vI}^{1}$.

ditzaquela ditzaqueela $\mathrm{G} \mathrm{VI}^{\mathbf{2}} 40$ L $\mathrm{LV}^{\prime}$.

Ipizpicu Ipizcu G $\mathrm{VI}^{1} 14 \quad \mathrm{Lv}^{\prime}$.

XXVII).

Kurz vor ditzaque(e)la steht ( $\left.\mathrm{G} \mathrm{VI}^{\prime} 20\right)$ degaquela; ich habe nicht untersucht was bei $L$. das Regelmässige ist. Er hat dailequeen $399^{1} 18$, gailezquee $\mathrm{F} \Pi^{1} 20$, duqueela $423^{1} 17$, luqueela "vI'11, guintuqueela A vI'17, dituqueeno $311^{125}$, aber anderseits guẽtezquelaco $262^{1} 20$, gaitezqueno $343^{\prime} 13$, degaqueno, daitezqueno, Uitezqueno u. 8. w.

Manche Vertretungen einer Wortform durch eine andere laxsen sich schwer unter einen bestimmten Gesichtspunkt bringen, so:

exercitatzeaz $=$ exercitzeaz $\mathrm{G} \mathrm{vI}^{\prime} 11=\mathrm{Lv}^{\mathrm{r}}$.

Die beiden lateinisch-romanischen Verben exercere und exercitare leben im heutigen Baskischen fort; dieses mit Anpassung des $k n$ an die baskische Lautierung: etsersitatzen, jenes mit Anschlues an die spanische Aussprache: equersitzen (doch wird auch etserçatzen gebraucht). L. Fcheint das Pz. exercitatu zu begünstigen $\left(389^{\prime} 14.399^{\prime} 11.417^{\prime} 14\right)$; doch exerci egac $371^{\prime} ?$ (glcich darauf exercitatione). Vgl. oben S. LXXVI: deithatzen neben deitzen. $\mathrm{Zu}$ :

gorputzeco = gorputzezco $\mathrm{CvI}$ '25 = CViu', von denen jenes gewiss im Allgemeinen das Häufigere ist, vgl. bihotzeco und bihotzezco u. A. S. LXXVI. Am Wenigsten versteht man:

huzcurria = hacitzea $\operatorname{EvI}^{\circ} 9=\mathrm{GI}^{*}$, 
CX

da jenes allen nordpyrenäischen Mdd. gemein ist; sollte $\mathrm{L}$. den Ausdruck für zu niedrig gehalten haben?

Ein syntaktischer Schnitzer ist verbessert worden: dugun $=$ ditugun $\mathrm{EvIII}{ }^{\circ} 19=\mathrm{GrV}^{1}$;

es folgt ja der Plural : gauça guciéc. Auch Dechepare ist in diesen Fehler verfallen, wie Dodgson "Euskara ${ }^{4}$ S. 88 bemerkt: Nic cugatic dudan penec (EIV ${ }^{*}$ ), wo der Vers die Verbesserung in ditudan nicht zulässt. Umgekehrt verhält es sich mit:

$$
\underset{\text { corruptionea }}{\text { gatic }}=\underset{\text { gatic }}{\text { corruptioneac }} \cdot \mathrm{G} \mathrm{vi}^{\mathrm{r}} 38=\mathrm{LIV}^{1} \text {, }
$$

da der Plural diraden = dirén vorhergeht; allein hier ist wohl die Leichtigkeit mit der in der Aussprache $\cdot c g$. und.$g$. verwechselt wurden, das Entscheidende. In guri gucioy-ere $=$ gurigucier-ere $\mathrm{D} \mathrm{II}^{1} 19=\mathrm{DVII}^{1}$ ist an Stelle des Artikels der 1. P. der der 3. P., der gewöhnliche getreten, vielleicht nach Massgabe der -er-Mundart (doch habe ich an andern Stellen, z. B. FI ${ }^{r} 33$ : hauc gucioc keine entsprechende Vertretung angemerkt).

Ausgefallenes ist eingeseizt worden, so:
1. Igandea
$\mathrm{B} \mathrm{VIII}^{\mathrm{r}}$ 9 R. $=\mathrm{B} \mathrm{VII}^{\mathrm{r}}$.
Miniftrea.
DVIII ${ }^{1} 5 / 6$ EvIII.

Zur Verdeutlichung des Textes dient die Einschaltung eines Wortes:
Segurago
$=$ heçaz Jegurago $\mathrm{FI}^{\mathrm{r}} 20$
$=\mathrm{G} \mathrm{vi}$.

Der franz. Text hat: "nous en rendre plus certains“", nämlich von den "promesses“; ; Vinson übersetzt unrichtig: „nous plus sûrs d'eux".

$$
\text { duté =duté hura } \mathrm{F}_{\mathrm{II}}{ }^{1} 39=\mathrm{H}^{\mathrm{r}} \text {. }
$$

Im Abc sind einmal einige Buchstaben bis auf ganz schwache Spuren nicht zum Abdrucke gekommen:

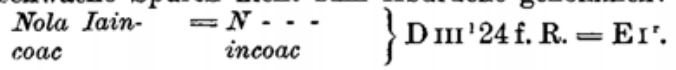

Eine Verschiebung ist eingetreten $\operatorname{Erv}^{r} 9$ R. $=\mathrm{FvI}^{r}$, sodass Iefus unmittelbar unter Luc. 11. 2 steht, ein paar Zeilen über Chriftec.

Eine Abkürzung ist aufgelöst.

$$
\text { H. }=\text { Haourra } \mathrm{BvIII}^{1} 36=\mathrm{BvIII}^{\circ} \text {. }
$$

Es versteht sich fast von selbst dass bei den verschiedenen Raumverhältnissen der beiden Texte die Abkürzungen der Randzitate vielfach anders ausgefallen sind: gala. $=$ gal., Sam. $=$ Sa., ephe. $=e p h$. u. s. w., wie ja diese Mannigfaltigkeit sich auch in jedem Texte für sich, sowie in dem des Neuen 
Testamentes zeigt; nur bemerke ich als wirklichen Druckfehler :

$$
\text { col. } \quad=\text { coll. } \quad \mathrm{GvI}^{\prime} 15=\mathrm{L} \mathbf{v}^{\mathbf{2}} \text {. }
$$

Was nun den zweiten Punkt anlangt, so würde er nur innerhalb der weiteren Untersuchung über die Sprache von L.8 andern Uebersetzungen gründlich erörtert werden können. Eine solche fällt aus dem Rahmen dieser Einleitung heraus, obwohl sie durch sie, wie ich denke, vorbereitet und erleichtert wird. Ich begnüge mich mit einer kurzen Bemerkung. L. hielt sich im Wesentlichen an das Westniedernavarrische, das heisst das was Pr. Bonaparte so nennt; denn dessen Sprachgeographie folge ich hier ubberall, nicht weil ich im Prinzlp ganz mit ihm einverstanden wäre, sondern weil so alle Unsicherheit ausgeschlossen wird. Dass L. die Mundart seiner Heimath, des Ortes Briscous, welcher politisch noch zum Labourd, sprachlich zum ostniedernavarrischen Gebiet gehört, begünstigt habe, lässt sich nicht erweisen. Er verfuhr, wie das die meisten baskischen Schriftsteller gethan haben, innerhalb gewisser Grenzen eklektisch. Seine Sorge dass auch die Souler sein Neues Testament verstehen möchten ergibt sich aus der diesem angehängten Verdolmetschung einer Reihe von Wörtern die den Soulern unbekannt waren ( $\mathrm{arI}$ ); übrigens scheint $\mathrm{er}$, mit Bestinmtheit lässt sich das natürlich nicht behaupten, manche soulische oder doch dem eigentlichen Labourdisch fremde Wörter (wie bathiric * $\mathrm{vI}^{1} 18$, haiçu F vIII'1) im TB gebraucht zu haben. Im Abc und im Kalender macht er den Soulern die Sprache noch etwas mundgerechter; wenn Vinson BB S. 44 daraus schliessen will dass diese Büchlein nur für die Soule und Niedernavarra bestimmt gewesen sind, so möchte ich doch fragen warum in diesem Falle dem Soulischen nicht noch mehr Zugeständnisse gemacht worden sind. Denn die thatsãchlich gemachten sind der Zahl und Art nach nicht bedeutend oder sogar zweifelhaft.

1) wird der Dat. Plur. statt auf -ey auf -er geendigt (das Demonstrativpronomen der 3 P.: hayer für h(ey); diesen Unterschied 2wischen Osten und Westen schenkt L. schon im Vokrabular eine besondere Aufmerksamkeit. Aber sogar Voltoire (1642), der der Mundart von Saint-Jean-de-Luz folgt, schreibt idiçagñer, vrdagñer, aftoçagñer, oullagñer eta arşagñer (S. 142; doch ahaidegusiey S. 204).

2) die auf rade ausgehenden Formen der 3. P. Plur. des Hülfsverbs "sein" werden durch solche auf $-r a$ ersetzt, so dirade = dira. Hier handelt es sich um die Einführung von überall Verbreitetem an Stelle von räumlich Beschränktem 


\section{CXII}

dirade neben dira kommt heutzutage gerade in der Soule (und in Guipuzcoa) vor; dass es hier nicht ganz jung ist, lässt das drade neben dra des Roncalischen von Urzainqui vermuthen (VB S. XXVIII). Uebrigens findet sich einige Mal sowohl dirade als dira in beiden Texten:

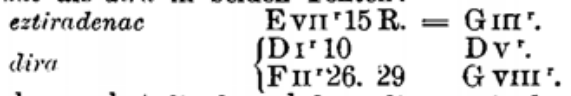

Dechepare hat dirade und dira, dire - ciraden und ciren. Vgl. Voltoire (1642) : diradé S. 173 neben sonstigem dire und dira. In der 1. P. Pl. des Präsens ist auch im TB gara die regelrechte Form, also z. B. baicara $\mathrm{DI}^{\mathrm{r}} 7=\mathrm{DrV}^{\prime}$; aber doch (vgl. S. LXXII):

baicarade $=$ baicara $\quad \mathrm{F}$ VIII $9=\mathrm{KIII}^{\mathrm{r}}$.

Ebenso herrscht als 1. P. Pl. des Imperfekts guinen (guenen); daneben kommt wie a. a. $O$. verzeichnet ist, ein parr $\mathrm{Mal}$ guinaden vor, und dieses ist sogar einmal in das Abc übergegangen: guinadelaric $\mathrm{FIII}^{\mathrm{r}} 35=\mathrm{G} \mathrm{vIII}^{r}$.

3) das Suffix -taco ist durch -çat ersetzt worden in:
Elicurendaco $=0$
giciarendaco
harendaco

Eliçarençat
Evi ${ }^{1} 10$
$=\mathrm{GII}{ }^{\mathrm{s}}$.
F II 28
GII ${ }^{1} 38$
G VIII ${ }^{\text {r. }}$
K VII'.

Wie sich dieser letzte Fall mundartlich verhält, habe ich nicht untersucht. Wenn L.

$$
\text { dirogu }=\text { decaquegu } \quad \mathrm{C} \mathrm{viII}^{r} 1=\mathrm{D} \mathrm{III}^{1}
$$

(Vinson übersetzt die Stelle: „nous ne pourrions le supporter“ statt "nous ne pouvons l'apporter"), so ist gerade die erste Form die heute dem Soulischen und Niedernavarrischen eigene, sie oder ihr zugehörige werden übrigens von ihm auch sonst im TB oft genug angewendet. Pr. Bonaparte verzeichnet in seiner Tabelle der L.schen Verbalformen (BB 587): diro, diroque, liro, liroque, ciroen, ciroqueen. Van Eys VAL S. 44 Anm. 1 setzt irrigerweise diroqueçue $179^{\circ} 43=$ drauqueçue.

Die Zahlen der Randzitate stimmen fast durchgängig in beiden Texten; nur:
Ioun. 3.81 eta $34=1$. Ioi. 4. 34 . (falsch)
Act.2.2Seta.4.27 Act.2.23,24,2\%.(richtig)
Rom. 8. 15
Rom. 5. eta 8. 15.
$\mathrm{GI}^{1} 15 \mathrm{f} .=\mathrm{Kv}^{\mathrm{r}}$.
GII'36 f. KVIr.
(rom....)4, 3,26 (Rom....) 4, s, 25 (richtig)
Grv ${ }^{1} 1 . \quad \mathrm{Lr}^{1}$.
Grv'6. LI'.

Ifat. 18. 17. 1. cor. 5. 6. 1. tim. 1. 20 (fälschliche Wiederholung von $\mathrm{G} \mathrm{viI}^{\mathrm{r}} 6 \mathrm{ff}$.) $=1$. Pier 5 1. cor. 14.40 (richtig) $\mathrm{G}$ vi ${ }^{1} 33 \mathrm{ff}$. $=\mathrm{Lv}^{\prime}$.

Das Trema ist hinzugesetzt worden: 
CXIU

$$
\begin{aligned}
& \text { ceruetarac - ceruëtara Cvi'7R. = Cvmri } \\
& \text { cervetarat ceruëtarat } \mathrm{CVI}^{\mathbf{1}} 12 \text { CVII'. }
\end{aligned}
$$

Umgekehrt:

Ceriëtan Ceruetan Erv'35 Fvir'.

Von grosser Wichtigkeit sind für uns die Aenderungen in der Akzentsetzung; sie würden von noch grösserer sein, wenn sich nicht neue Inkonsequenzen oder Nachlässigkeiten eingemischt hätten. Von den Fällen in denen in beiden Texten der Akzent wider die Regel fehlt, sehe ich ab. Ich folge der oben gegebenen Eintheilung und setze bloss die Schreibung des TB.

1) a) a) as): Im TB fehlt der Akzent auf dem $a$, im Abc

\begin{tabular}{|c|c|c|}
\hline $\begin{array}{l}\text { mifericordia } \\
\text { hifloriaz } \\
\text { Eliçaz } \\
\text { obrac } \\
\text { hifloria } \\
\text { Refumaz } \\
\text { Elican } \\
\text { penac } \\
\text { caufa } \\
\text { abundantia } \\
\text { ceremonia } \\
\text { compainia } \\
\text { Eligan }\end{array}$ & 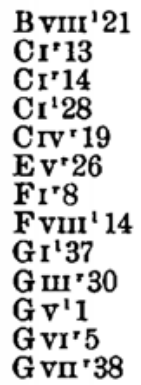 & 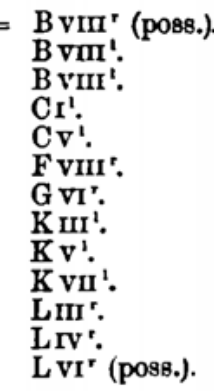 \\
\hline
\end{tabular}
ist er richtig gesetzt:

Im Abc falsch gesetzt:
amorioaz
B VII ${ }^{1} 22$
B vir' ${ }^{\prime}$

In andern Fällen steht der Akzent im TB richtig, fehlt im

\begin{tabular}{|c|c|c|}
\hline $\begin{array}{l}\text { manerá } \\
\text { prouidentióz } \\
\text { Taulán } \\
\text { gratiá }\end{array}$ & $\begin{array}{l}\text { B vIII' } 29 \\
\text { CI'29 R. } \\
\text { DII'38R. } \\
\text { DIII'30 }\end{array}$ & $\begin{array}{l}\text { BvII'. } \\
\text { CI'. } \\
\text { DvI' } \\
\text { EI' (poss.; aber es folgt } \\
\quad \text { eta amorioa). }\end{array}$ \\
\hline $\begin{array}{l}\text { obrác } \\
\text { arimác } \\
\text { obedientiáz }\end{array}$ & $\begin{array}{l}\text { DVI } 10 \\
\text { DVII } 19 \\
\text { EI'19 }\end{array}$ & $\begin{array}{l}\text { Erv'. } \\
\text { Evi'. } \\
\text { Fin'. }\end{array}$ \\
\hline requefii & $\left\{\begin{array}{l}\mathrm{Ev}^{\mathbf{2} 25} \mathrm{R} . \\
\mathrm{E} \mathbf{v}^{\circ} 4 \mathrm{R} .\end{array}\right.$ & $\begin{array}{l}\text { Fviri }{ }^{5} \text {. } \\
\text { GI'. }\end{array}$ \\
\hline $\begin{array}{l}\text { Eguiá } \\
\text { perfonán } \\
\text { dependentiá }\end{array}$ & $\begin{array}{l}\text { FI' } 32 \mathrm{R} . \\
\text { GII'39 } \\
\text { G VII'10 }\end{array}$ & $\begin{array}{l}\text { G viII'. } \\
\text { K vir'. } \\
\text { Lv' }^{\prime} \text { (poss.; und es geht } \\
\quad \text { vorher forma). }\end{array}$ \\
\hline
\end{tabular}
Abc: 


\section{CXIV}

bb) Im TB fehlt der Akzent auf $\operatorname{dem} e$, im Abc ist er richtig gesetzt:
vicien
Deabruez
vicien
$\mathrm{CI}^{\mathrm{x}} \mathrm{2}$
CII 50 R.
$\mathrm{FvII}^{1} 24$
CiI'.
vicien-ere
F viri' 3
$\mathrm{K} \mathrm{II}^{\mathrm{r}}$.
bckatuen
G VI' 10
K III'.
LIV ${ }^{r}$.
hilén
$\mathrm{EII}^{\mathrm{r}} 14 \mathrm{R}$. $=\mathrm{FIII}^{r}$.
Sacramenduéz
$\mathrm{FI}^{\mathrm{r}} 17 \mathrm{R}$.
G VIr.
facramẽduéz
$\mathrm{F}$ II ${ }^{\mathrm{r}} 10 \mathrm{R}$.
G viI'.

$=\mathrm{BvII}^{\boldsymbol{r}}$ (es folgt hilén).

Im TB steht der Akzent richtig und fehlt im Abc:

Dem -ey des TB (nur ausnahmsweise betont, so Iuduéy DV'13) entspricht regelmässig ér im Abc: etfayey = etfayér, fideley $=$ fidelér u. s. w.; doch findet sich dies auch einige Male ohne Akzent, so gucier $\left(\mathrm{CvI}^{1} 13=\mathrm{D} \mathrm{I}^{\mathrm{r}}\right.$, dariztener $\left(\mathrm{D} \mathrm{III}^{1} 38 \Rightarrow\right) \mathrm{EI}^{1}$, dituztener $\left(\mathrm{D}_{\mathrm{II}}{ }^{1} 39=\mathrm{EI}^{1}\right.$, hayer $\left(\mathrm{DVI}{ }^{\prime} 17=\right) \mathrm{EV}^{\boldsymbol{r}}$.

dieçán

3) Im TB steht der Akzent richtig und fehlt im Abc: $\mathrm{EVI}{ }^{\mathrm{r}} 10=\mathrm{GI}$.

b) a) Im TB fehlt der Akzent, im Abc steht er richtig: Iaincoa $\mathrm{Cv}^{\mathrm{r}} 15=\mathrm{C} \mathrm{vII}^{*}$ (unmittelbar vorher richtig Iaincoá auch im TB).

c) a) Im TB steht der Akzent richtig, und fehlt im Abc: dituún $\mathrm{BviII}^{1} 5=\mathrm{BvII}^{\mathrm{i}}$.

d) a) Ebenso:

dugunçát

G VII ${ }^{1} 10 \quad$ LVI'.

f) Jene Fälle in denen das TB den Akzent sicher oder vermuthlich in Folge eines Versehens auf der letzten Silbe hat, sind zum Theil im Abc unverändert geblieben, so principalá - delá (zwei Mal) - eguitecotán, halatán (zwei Mal), nolatán - harén - ecén - eguitén. Aber zum Theil ist der Akzent beseitigt worden: denaz, harçaz - repoftaren - detefratzen eman (zwei Mal; ein Mal emã), und auch ein Mal halatan (Cv'22 $=\mathrm{CVIII}^{\mathrm{r}}$ ). Ebenso in haourretan, wofür haourrétan $\mathrm{zu}$ erwarten war. Ferner ist zu bemerken:

$$
\text { bere }=\text { beré } \quad \mathrm{Fv}^{\mathrm{r}} 26=\mathrm{Hrv}^{1} \text {. }
$$

2) c) Der falsche Akzent in eternála, Iaincoáren im TB ist beseitigt worden im Abc. Anderseits ist er hier falsch gesetzt:

$$
\begin{aligned}
& \text { deşançat }=\text { deçánçat } \mathrm{DIV}^{1} 31=\mathrm{E}_{\mathrm{H}^{1}} \text {. } \\
& \begin{array}{llll}
\text { dela } & \text { déla } & \mathrm{F} \text { vir }{ }^{\mathrm{2}} 28 & \mathrm{KII}^{*} \text { (vgl. oben }
\end{array} \\
& \text { delá); }
\end{aligned}
$$

jenes zweifellos für decançát.

3) Der falsche Akzent in bércetan im TB ist im Abc 
richtig gesetzt worden: bercétan, der von ézgaituztela getilgt. Hingegen steht im Abc der Akzent falsch:

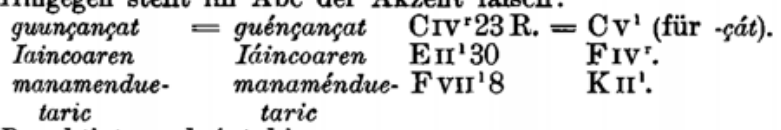

Berechtigt erscheint hier :

duanean $=$ duánean $\mathrm{CI}^{1} 18=\mathrm{Cr}^{1}$.

Der Bindestrich ist im Abc gesetzt worden, wo das TB ohne ihn zwei Wörter hat:

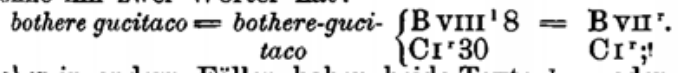

aber in andern Fällen haben beide Texte $b .-g$. oder beide $b . g$. Ebenso:

lurreãere = lurrean-ere $\mathrm{Erv}^{*} 11=\mathrm{FvI}^{\mathrm{r}}$;

gleich darauf guc ere in beiden Texten, higui-|ere = higuiere $\mathrm{CII}^{\prime} 8=\mathrm{CII}^{r}$. Falsch gesetzt ist der Bindestrich, durch den folgenden Theilungsstrich veranlasst, im Abc:

badere eztu = badere-ez-itu $\mathrm{EII}^{2} 20=\mathrm{F} \mathrm{III}^{2}$.

Der Bindestrich ist, dem allgemeinen Gebrauch zufolge, beseitigt in:

mifericor : $=$ mifericordiaga- GIII'22 $=\mathrm{K} \mathrm{VII}^{1}$. dia-gatic tic

Die Interpunkzion ist im Abc an sehr vielen Stellen und zwar im verschiedensten Sinne verbessert worden; an manchen ist das Fehlerhafte geblieben, an etwas zahlreicheren sogar erst eingetreten. Um nur von den dritten Fällen zu reden, so ist, ich setze bloss die Schreibung des TB, weggelassen der Doppelpunkt $\left(E z\right.$ : ecen D VII $\left.{ }^{r} 2=\mathrm{EVI}^{r}\right)$, der Beistrich (gloriá, erraiten $\mathrm{D} \mathrm{IV}^{1} 23=\mathrm{EII}^{1}$; baicen, baina $\mathrm{Er}^{\mathrm{r}} 37=\mathrm{F}_{\mathrm{II}^{2}}{ }^{2}$; hinzugefügt der Beistrich (ahal ceçaqueagu $\mathrm{CvII}^{1} 35=\mathrm{D} \mathrm{III}^{r}$; Baldin Iaincoaz $\mathrm{Drv}^{1} 12=\mathrm{EII}^{1}$; goiti alchatzen $\mathrm{Ev} \mathrm{v}^{r} 1=\mathrm{F} \mathrm{VII}^{1}$; içateco feignalea $\mathrm{Fv}^{\prime} 25 \mathrm{R} .=\mathrm{HV}^{r}$ ); gesetzt der Punkt statt des Fragezeichens (dirade $=$ dira. $\mathrm{E}_{\mathrm{II}^{\mathrm{r}}} 37=\mathrm{F} \mathrm{III}^{\mathrm{l}}$ ), statt des Beistrichs (dituc, Egunecoa $\mathrm{EvI}^{\mathrm{r}} 29=\mathrm{GI}^{1}$; da, Moyfesec $\mathrm{F} \mathrm{III}^{\mathrm{r}} 34$ $=\mathrm{H}^{1}$ ), der Doppelpunkt statt des Punktes (baitzaicu. Ecẽ F IV' $\left.16=\mathrm{H}^{\prime} \mathrm{III}^{\mathrm{l}}\right)$.

Zum Schlusse erwähne ich noch einige ganz nebensächliche Verschiedenheiten zwischen dem TB und dem Abc. Im letzteren sind an die Stelle der Sterne, welche, da die des Randes öfter ziemlich weit von den entsprechenden des Textes entfernt sind, leicht Verwirrung anstiften können, Buchstaben getreten, und zwar auf jeder Seite von $a$ an. Die Minuskeln des TB sind im Abc ein paar Mal zu Majuskeln geworden; baina $=$ 


\section{CXVI}

Baina $\mathrm{DI}^{1} 36=\mathrm{D} \mathrm{vI}^{1}$ ist selbstverständlich, auch taulatan -aren, -arena $=$ T. $\mathrm{D} \mathrm{II}^{\circ} 34.36 .38=\mathrm{D}_{\mathrm{VII}}{ }^{\circ}$ (Taulán auch im TB $\mathrm{D}^{\circ} 37 \mathrm{R}$.), Aduocat eta ararteco $=$ A. eta $A$. $\mathrm{G}^{\mathrm{*}} \mathbf{1 9}=$ LII' (Ararteco auch im TB EII'4. GIV'5) haben ihre Berechtigung, aber $\mathrm{zu}$ interceditzen eta aduocatzen $=I$. eta $A$. E II ${ }^{1} 19=$ L II $^{1}$ hat offenbar das Aduocat des Vorhergehenden verführt (vgl. Euangelizatzen oben S. XXIII). Das $M$. von EvIIr $^{r} 38$ im TB ist, wie sich gehört, im Abc zu Anfang der folgenden Zeile gesetzt.

H. Schuchardt.

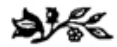

\section{Verbesserungen und Nachträge.}

*II'23. Lies est statt eft.

XV, 11. Lies baitaye statt bataye.

XVII, 5. Auch $\mathrm{AvI}^{\mathrm{r}}, \mathrm{Fr}^{1}{ }^{1}, \mathrm{G}_{\mathrm{VI}}{ }^{r}$ haben 40 'Zeilen; hingegen $\tilde{\mathrm{i}} \mathrm{III}^{1}, \mathrm{IV}^{r} 49$ statt 48 , anderseits $\tilde{\sigma} \mathrm{VI}^{1}, \mathrm{VII}^{r}, \mathrm{VII}^{1}$, VIII ${ }^{r}$ 47 statt 48 .

XXVนI, 37. Füge hinzu: cieçén $3^{\mathrm{r}} 8$.

XXX, 26. Lies ikhusi statt ihkusi (nachträglich verdruckt).

XXXI, 2 f. Füge hinzu: Ioannic $114^{1} 22$ (es folgt bald Ioannefi). Hierher hä̈te auch BBaina von LXVI, 33 gehört. druckt).

XXXI, 5. Lies ikufßiric statt ikuifßric (nachträglich ver-

XXXI, 20a. Lies ${ }^{63}$ statt ${ }^{66}$.

$\mathbf{X X X V}, 36$ ?. Füge hinzu: gaiçoac $\mathrm{Bv}$ r9.

XXXIX, 12. Bei L. findet sich, ebenso wie bei andern Schriftstellern, nicht selten -ric für -tic; so Italiaric 401' Schl., Macedoniaric $243^{\prime} 5$ u. s. w., Romaric $339^{*}$ Schl. u. s. w. neben Ephefetic, Galileatic, Ierufalemetic, Indeatic u. s. w.

XXXIX, 35 f. b. Füge hinzu: diffama- $2^{\times} 19.247 \times 37$. F VII ${ }^{\circ} 26$. VIII ${ }^{\circ} 19$.

XL, 26 a. Lies albeitzinteizte statt -eitze.

$\mathbf{X L}$, 34. Lies guinten statt ginten.

XL, 39 ^. Baitaiz ist wohl regelmässig; ebenso gebildet ist albeitindoa $8^{1} 41$.

XLVI, 41 b. Contrataco Fvir' 31 .

XLVII, 3. Lies $\mathrm{I}^{\mathrm{r}} 31$ statt $\mathrm{I}^{\mathrm{r}} 31$. 